\title{
MASS BALANCE: A KEY TO ADVANCING MONITORED AND ENHANCED ATTENUATION FOR CHLORINATED SOLVENTS
}

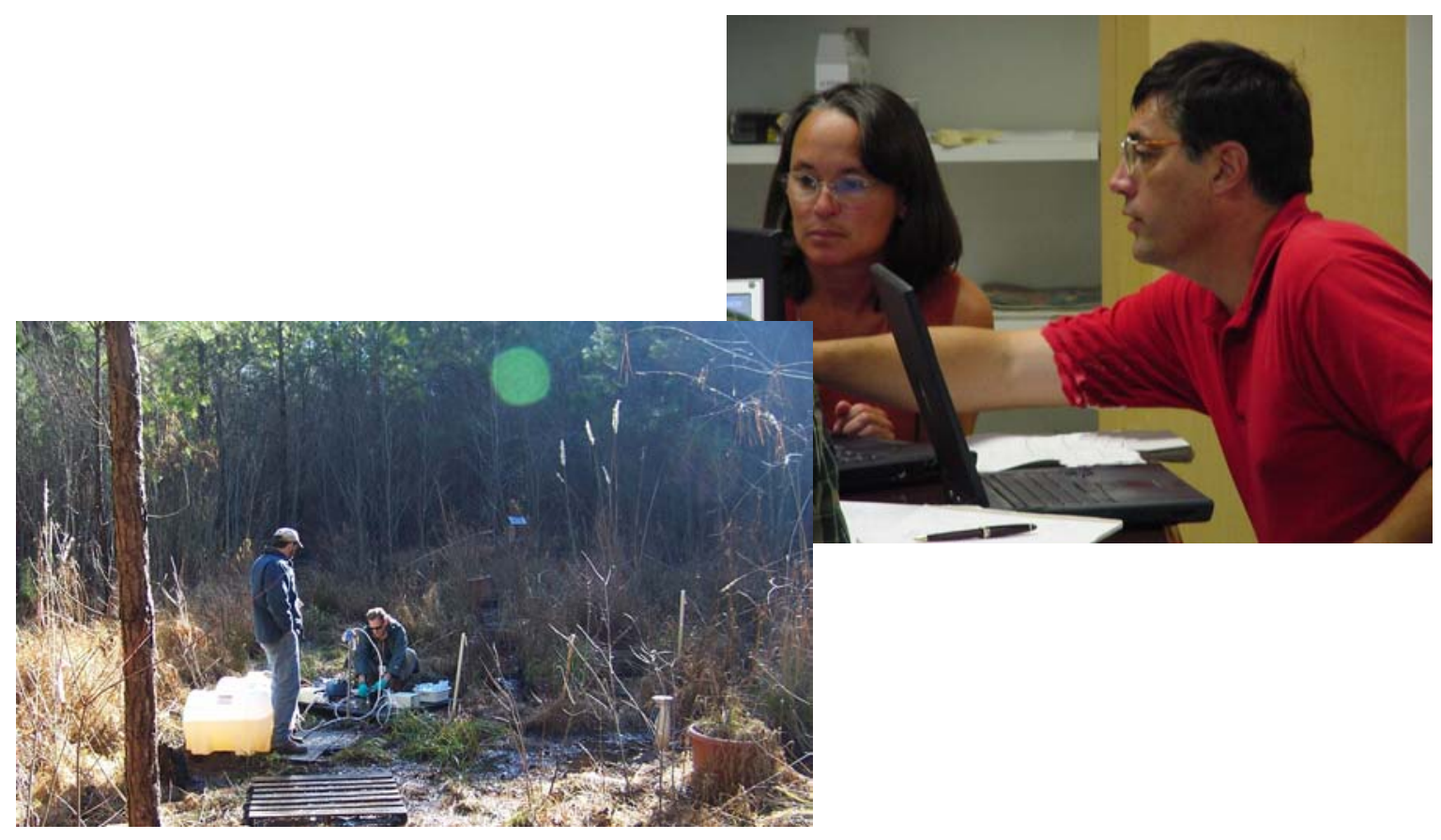

JUNE 2006

Washington Savannah River Company Savannah River Site

Aiken, SC 29808

Prepared for the U.S. Department of Energy Under Contract Number DEAC09-96-SR18500

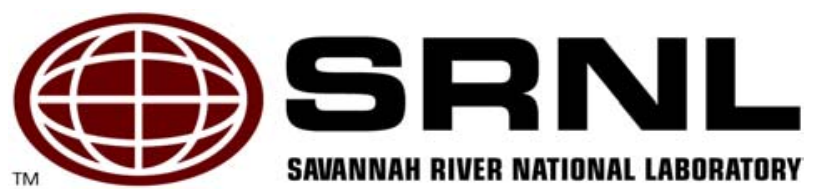




\section{ACKNOWLEDGEMENTS}

We would like to acknowledge those who played a key role in the development of this work. The United States Department of Energy (US DOE) Office of Cleanup Technologies sponsored this effort. We appreciate the guidance and support of Claire Sink of US DOE Headquarters and Karen Adams of US DOE Savannah River. We acknowledge the participation and collaboration of other federal agencies, notably, the U.S. Geological Survey (USGS) and the U.S. Environmental Protection Agency (US EPA), as well as state agencies such as the SC Department of Health and Environmental Control, the FL Department of Environmental Protection, and the multi-state Interstate Regulatory and Technology Council. We are grateful to the independent peer review panel for their careful reviews and helpful input.

\section{DISCLAIMER}

Preparation of this report was coordinated by Washington Savannah River Company (WSRC) for the United States Department of Energy (DOE) under Contract No. DE-AC09-96SR18500. Extensive effort was made by the authors to assure the accuracy of the contents and interpretation. However, neither the DOE, nor WSRC, nor any of their employees makes any warranty, express or implied, or assumes any legal liability or responsibility for the accuracy, completeness, or usefulness of any information, apparatus, or product, or process disclosed herein, or represents that its use will not infringe privately owned rights. Reference herein to any specific commercial product, process, or service by trademark, name, manufacturer, or otherwise does not necessarily constitute or imply endorsement, recommendation, or favoring of same by the United States Government or any agency; thereof. The views and opinions of the authors expressed herein do not necessarily state or reflect those of the United States Government or any agency thereof.

\section{Printed in the United States of America}

Prepared For U.S. Department of Energy 
Key Words:

remediation

environment

Retention:

Permanent

\title{
MASS BALANCE: A KEY TO ADVANCING MONITORED AND ENHANCED ATTENUATION FOR CHLORINATED SOLVENTS
}

\author{
Brian B. Looney, Savannah River National Laboratory \\ Francis Chapelle, U.S. Geological Survey \\ Tom O. Early, Oak Ridge National Laboratory (retired) \\ Karen M. Vangelas, Savannah River National Laboratory \\ Karen M. Adams, U.S. Department of Energy \\ Claire H. Sink, U.S. Department of Energy
}

\section{JUNE 2006}

Washington Savannah River Company Savannah River Site

Aiken, SC 29808

Prepared for the U.S. Department of Energy Under Contract Number DEAC09-96-SR18500
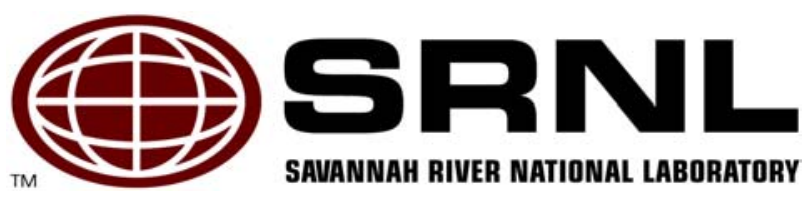
WSRC-STI-2006-00082, Rev. 0 June 8, 2006

Page iii of xiii

This page intentionally left blank. 
In 2003, the U.S. Department of Energy (DOE) Office of Environmental Management authorized an alternative project at the Savannah River Site designed to develop cost-effective and environmentally protective technical solutions for the challenge of large and complex plumes of chlorinated volatile organic compounds (cVOCs) at DOE sites. The project builds upon the current U.S. Environmental Protection Agency (EPA) protocol and directive (EPA, 1998 and 1999) for monitored natural attenuation (MNA). The DOE effort is built upon three major technical thrust areas: mass balance, enhanced attenuation (EA), and innovations in characterization/monitoring. Each of these three topics is being supported by a broad-based group of recognized technical experts and each group is currently developing an interim report related to the topic area. By introducing the concepts of mass balance and EA, along with the technical basis and documentation for these concepts, the project is working toward a goal of providing new and powerful tools for transitioning from active remediation to a protective, long-term monitoring state. This project is a departure from classical MNA in that its central theme is to take an active part, as needed, to achieve a favorable balance between the release of contaminants from sources (source loading) and processes that destroy or retard migration of contaminants in resultant plumes (attenuation capacity of the system).

\section{OBJECTIVE}

This interim technical report provides the technical basis for the mass balance concept, to summarize relevant case studies from the literature and ongoing research efforts. The overall objective is to demonstrate that a mass balance paradigm is a powerful framework that provides information and the technical basis for attenuation based remediation.

\section{ORGANIZATION OF THE REPORT}

This report introduces the mass balance concept, examines the contributions and significance of various components of the mass balance, and provides an initial assessment of promising development areas. This information is provided in eight major sections and two appendices.

Section 1 is the executive summary.

Sections 2 and 3 define and describe the mass balance idea in general terms. Importantly, these sections discuss the centrality of this concept when the ultimate goal is determining plume stability. These sections also define key concepts such as "empirical" and "deterministic" as they relate to the methods to assess mass balance, and these sections introduce the various processes that contribute to mass balance.

Sections 4 and 5 examine the empirical and deterministic assessment approaches in more detail and describe how these approaches can work together.

Section 6 is a parametric study that examines the relative significance of various site specific factors in contributing to plume stability - the section is structured so the various processes are layered in one at a time to allow the reader to observe and compare the relative impacts. 
Sections 7 and 8 summarize the evaluation in terms of research and development directions and broad goals.

Section 9 provides the references for the report.

Finally, the appendices provide supplemental information and mathematical derivations to support Sections 4 and 6. The appendices are intended to provide sufficient detail to allow interested readers to better follow and replicate the evaluation and to facilitate technical review and discussion.

The process of systematically disassembling and then reassembling a "contaminant plume"in terms of loading and attenuation capacity - provided useful insights and promising directions to specifically improve implementation of natural attenuation, and generally improve environmental management and legacy management decision-making. 


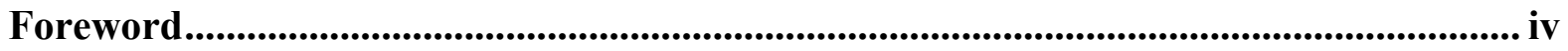

Objective ...................................................................................................................................... iv

Organization of the Report .......................................................................................................... iv

List of Figures.................................................................................................................................. vii

List of Tables ...................................................................................................................... viii

List of Case Studies......................................................................................................................... viii

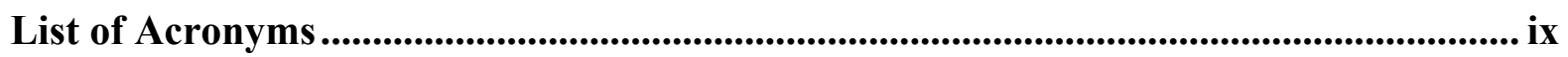

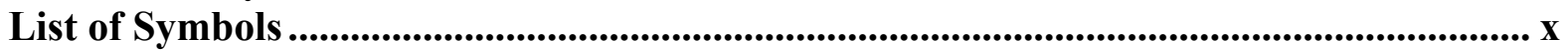

Key Definitions ............................................................................................................................ xii

1.0 Executive Summary ........................................................................................................ 1

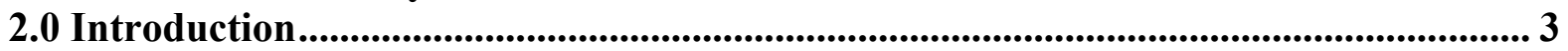

3.0 Processes Contributing to Attenuation for Chlorinated Solvents ................................... 9

4.0 Alternative Approaches to Quantify Mass Balance......................................................... 13

4.1 Developing Mass Balance Equations .................................................................... 14

4.2 Summing the Processes Contributing to Natural Attenuation................................... 18

4.3 Time of Remediation ..................................................................................................... 18

5.0 Integrating the Empirical and Deterministic Approaches to MNA.............................. 21

6.0 Modeling - Reassembling the System ......................................................................... 23

6.1 Interaction of Sorption and Degradation ............................................................................. 27

6.2 Effects of Dispersion and Source Decay - Method Development .............................. 30

6.3 Effects of Longitudinal Dispersion................................................................................ 33

6.4 Effects of Source Decay ....................................................................................................... 36

6.5 Summary of the Results for 1D Cases ................................................................................ 39

6.6 Overlay Effect of Transverse Dispersion....................................................................... 39

6.7 Summary of Bounding Cases ................................................................................................ 41

7.0 Mass Balance Research Needs and Research Support..................................................... 51

8.0 Moving Toward a Mass Balance Approach ........................................................................ 55

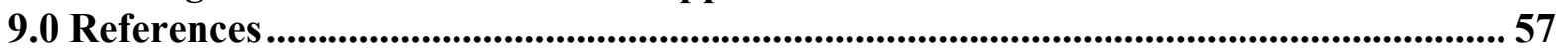

Appendix A. Supplemental Information - Derivation of Transport Equation................ 63

Addendum for Section 4.1 ........................................................................................................ 63

Appendix B. Supplemental Modeling information for Parametric Evaluation.............. 69

Addendum for Section 6.2 - A more detailed discussion of Equation 3........................ 69

Addendum for Section 6.3 - Mathematical formulation used to evaluate longitudinal dispersion ................................................................................................. 70

Addendum for Section 6.4 - Mathematical formulation used to evaluate source decay ................................................................................................................... 71

Addendum for Section 6.6 - Mathematical formulation used to evaluate transverse dispersion .................................................................................................................... 73

Addendum for Section 6.6 - Graphs of the results for the Medium and Large Site Cases showing the effects of transverse dispersion are on the next two pages. 


\section{LIST OF FIGURES}

Figure 2-1. The four stages describing the development of a contaminant plume (adapted from Newell and Conner 1998).................................................................................... 3

Figure 2-2. Steady-state mass balance example for water in a subsurface system................. 4

Figure 2-3. Steady-state mass balance example for contaminants in a subsurface system .... 4

Figure 2-4. Simplified steady-state contaminant mass balance block diagrams for a

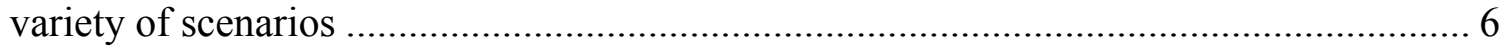

Figure 3-1. Graphical depiction of natural attenuation mass balance..................................... 11

Figure 3-2. Graphical depiction of enhanced attenuation mass balance................................ 12

Figure 4-1. The Empirical Approach to Describing the Mass Balance of a System Depends on Site-Specific Monitoring Data ......................................................................... 13

Figure 4-2. Typical "control volume" used to develop mass balance equations for

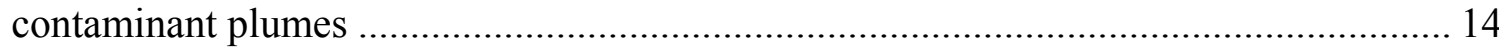

Figure 5-1. A Process for Integrating the Empirical and Deterministic Approaches for

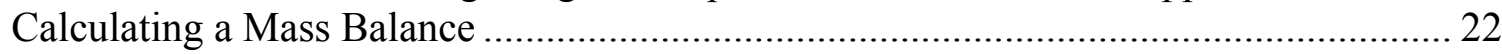

Figure 6-1. Plume size and structure for a 1D plume influenced only by degradation and

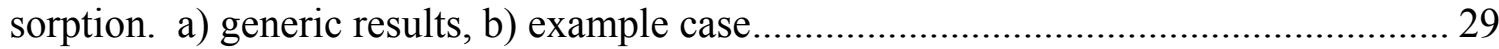

Figure 6-2. General Geometry and Nomenclature for Analytical Solution........................... 32

Figure 6-3. Plume size and structure for a 1D plume influenced by degradation, sorption,

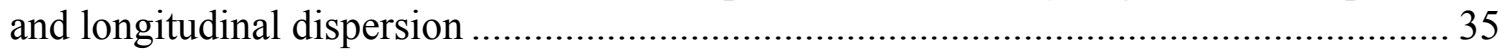

Figure 6-4. Plume size and structure for a transient 1D plume influenced by source decay, degradation, sorption, and longitudinal dispersion ............................................. 37

Figure 6-5. Normalized graph showing plume size and structure for a 1D plume under different assumptions about the source and longitudinal dispersion ............................... 40

Figure 6-6. General impact of transverse dispersion on centerline plume concentration - scaled attenuation contribution versus flow distance

Figure 6-7. Small Site -- Overlay of the attenuation by transverse dispersion for a 2D steady-state plume

Supplemental Figure. Medium Site -- Overlay of the attenuation by transverse dispersion for a $2 \mathrm{D}$ steady-state plume.

Supplemental Figure. Large Site -- Overlay of the attenuation by transverse dispersion for a $2 \mathrm{D}$ steady-state plume. 


\section{LIST OF TABLES}

Table 6-1. Key Site Parameters that Influence MNA/EA................................................. 27

Table 6-2. Bounding values for remediation goals and the derived parameter $\left(\mathrm{L}_{\lambda}\right) \ldots \ldots \ldots \ldots . .28$

Table 6-3. Summary of Some of the Available Analytical Models...................................... 31

Table 6-4. Projected Steady-State or Maximum Plume Sizes for the Three Bounding

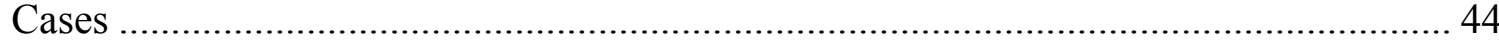

Table 6-5. Consensus Evaluation of Various Attenuation Mechanisms............................... 46

Table 6-6. Applicability of Various Attenuation Mechanisms to Typical Chlorinated Solvents

Table 7-1. Considerations for Selecting Mass Balance Evaluation Approach Based

On Site Conditions 52

Table 7-2. Selected Projects and Applicability to the Three Major Thrust Areas 53

\section{LIST OF CASE STUDIES}

Case Study 3-1. Innovations by State Regulatory Agencies.............................................. 8

Case Study 4-1. Measuring Flux - The Field Approach ............................................... 15

Case Study 4-2. Real-World Use of Flux as a Measure of MNA......................................... 16

Case Study 6-1. Using Field and Lab Data to Estimate Model Input.................................... 24

Case Study 6-2. A Zonation Approach to Heterogeneity ...................................................... 26

Case Study 6-3. What if Degradation Is Only in the Aqueous Phase? .................................. 34

Case Study 6-4. Determining the Value of Source Removal................................................. 38

Case Study 6-5. DAFfy Graphs: An Innovative Approach for Modeling ............................ 43

Case Study 7-1. Coupled Models in Action........................................................................ 54 


\section{LIST OF ACRONYMS}

\begin{tabular}{|c|c|}
\hline API & American Petroleum Institute \\
\hline CA & chloroethane \\
\hline $\mathrm{CF}$ & chloroform \\
\hline CT & carbon tetrachloride \\
\hline cVOCs & chlorinated volatile organic compounds \\
\hline DAF & dilution attenuation factors \\
\hline DCA & dichloroethane \\
\hline DCE & dichloroethene \\
\hline DCM & dichloromethane \\
\hline DOD & Department of Defense \\
\hline DOE & Department of Energy \\
\hline EA & Enhanced Attenuation \\
\hline EPA & Environmental Protection Agency \\
\hline $\mathrm{FeS}$ & iron sulfide \\
\hline FRAMES & Framework for Risk Analysis in Multimedia Environmental Systems \\
\hline GMS & $\begin{array}{l}\text { GMS groundwater modeling pre- and post-processor that supports 2D \& 3D } \\
\text { geostatistics, and both finite element and finite difference models in 2D \& 3D. }\end{array}$ \\
\hline GUI & graphical user interface \\
\hline iMF & integrated Mass Flux \\
\hline ITRC & Interstate Technology \& Regulatory Council \\
\hline LM & Legacy Management \\
\hline MNA & Monitored Natural Attenuation \\
\hline NAPL & non-aqueous phase liquid \\
\hline OSWER & (EPA) Office of Solid Waste and Emergency Response \\
\hline PCE & tetrachloroethene \\
\hline PDE & partial differential equation \\
\hline SCRD & State Coalition for Remediation of Dry Cleaners \\
\hline TCA & trichloroethanee \\
\hline TCE & trichloroethene \\
\hline
\end{tabular}




\section{LIST OF SYMBOLS}

\begin{tabular}{|c|c|}
\hline $\mathrm{A}_{\mathrm{c}}$ & Attenuation Capacity \\
\hline$A_{r}$ & Attenuation Rate, mass/volume/time \\
\hline $\mathrm{iA}_{\mathrm{r}}$ & integrated Attenuation Rate, mass / time. \\
\hline $\mathrm{B}$ & amount of cells, mass \\
\hline $\mathrm{C}$ & aqueous concentration, mass/volume \\
\hline $\mathrm{C}_{0}$ & source zone aqueous concentration at time zero, mass/volume \\
\hline $\mathrm{C}_{(\mathrm{x}, \mathrm{y}, \mathrm{z}, \mathrm{t})}$ & concentration at a specific target location and specified time, mass/volume \\
\hline Cs & $\begin{array}{l}\text { concentration of contaminant in solid phase, mass of contaminant / mass of } \\
\text { solid phase }\end{array}$ \\
\hline $\mathrm{D}$ & coefficient of dispersion, length ${ }^{2} /$ time $^{2}$ \\
\hline Discharge & the integrated mass flux exiting a specified volume, mass/time. \\
\hline & fraction organic carbon, dimensionless \\
\hline$f_{l s}$ & mole fraction of solute, dimensionless \\
\hline$f_{x}$ & $\begin{array}{l}\text { term accounting for advection, longitudinal dispersion and first order decay, } \\
\text { dimensionless }\end{array}$ \\
\hline$f_{y}$ & term accounting for dispersion in the lateral y direction, dimensionless \\
\hline$f_{\mathrm{z}}$ & term accounting for dispersion in the lateral $\mathrm{z}$ direction, dimensionless \\
\hline $\mathrm{k}_{\mathrm{s}}$ & $\begin{array}{l}\text { exponential coefficient describing the change in source zone concentration } \\
\text { (representing discharge) over time. }\end{array}$ \\
\hline $\mathrm{k}_{\mathrm{x}, \mathrm{y}, \text { or z }}$ & hydraulic conductivity, length/time \\
\hline $\mathrm{K}_{\mathrm{d}}$ & $\begin{array}{l}\text { distribution coefficient describing solute partition between the adsorbed and } \\
\text { dissolved state, volume of water / mass of solid, }\left(\text { i.e., } \mathrm{C}_{\mathrm{s}} / \mathrm{C}\right)\end{array}$ \\
\hline $\mathrm{K}_{\mathrm{oc}}$ & organic carbon distribution coefficient, dimensionless \\
\hline $\mathrm{L}_{\lambda}$ & $\begin{array}{l}\text { characteristic transport length controlling the steady-state plume size and } \\
\text { structure, length }\end{array}$ \\
\hline Loading & $\begin{array}{l}\text { the integrated mass flux entering a specified volume (e.g., from a former source } \\
\text { zone), mass/time }\end{array}$ \\
\hline M & mass of contaminant in a specified volume, mass \\
\hline $\mathrm{M}_{\mathrm{o}}$ & initial mass of contaminant in a specified volume, mass \\
\hline $\mathrm{M}_{\text {remaining }}$ & contaminant mass remaining at any time, mass \\
\hline $\mathrm{M}_{\text {threshold }}$ & contaminant mass at a given threshold, mass \\
\hline iMF & integrated mass flux, mass/time \\
\hline $\mathrm{n}$ & aquifer porosity, dimensionless \\
\hline $\mathrm{R}$ & retardation coefficient, dimensionless \\
\hline source & input rate of a constituent into the monitored system, mass / time \\
\hline $\mathrm{t}$ & time \\
\hline $\mathrm{t}_{\text {remediation }}$ & time of remediation, time \\
\hline $\mathrm{v}_{\mathrm{c}}$ & contaminant velocity, length/time \\
\hline $\mathrm{v}_{\mathrm{d}}$ & darcy velocity, length/time \\
\hline $\mathrm{v}_{\mathrm{s}}$ & groundwater seepage velocity, length/time \\
\hline $\mathrm{V}$ & volume, length ${ }^{3}$ \\
\hline $\mathrm{x}$ & travel distance, length \\
\hline
\end{tabular}


$\alpha$

$\alpha_{\mathrm{x}}$

$\alpha_{\mathrm{y}}$

$\alpha_{z}$

$\lambda$

$\lambda_{\mathrm{a}}$

$\rho_{\mathrm{b}}$

coefficient of dispersivity, length

coefficient of longitudinal dispersivity, length

coefficient of lateral transverse dispersivity, length

coefficient of lateral vertical dispersivity, length

$1^{\text {st }}$ order degradation rate or biodegradation rate constant, $1 /$ time

$1^{\text {st }}$ order degradation rate or biodegradation rate constant if all degradations is assumed to occur in the aqueous phase, 1/time

average bulk density of aquifer solids, mass/volume 


\section{KEY DEFINITIONS}

\section{Enhancement, Enhanced Attenuation}

An enhancement is any type of intervention in a contaminated system that decreases contaminant loading or increases the magnitude of attenuation by natural processes beyond that which occurs without intervention. Enhanced attenuation is the result of applying an enhancement that sustainably manipulates a natural attenuation process leading to an overall reduction in mass flux of contaminants.

\section{Integrated Mass Flux (iMF), Loading, Discharge}

The integrated mass flux is the total quantity of a migrating substance that moves through a planar transect that is within the system of interest and oriented perpendicular to the direction of movement. If the transect is at the entry point to the system the integrated mass flux is the loading. If the transect is at the exit point from the system, the integrated mass flux is the discharge. Note that these terms have units of mass per time (e.g., $\mathrm{Kg} / \mathrm{yr}, \mathrm{g} /$ day or the like) and they represent an extension of the traditional engineering definition of flux (e.g., $\mathrm{Kg} / \mathrm{yr} / \mathrm{m}^{2}$ ) in which the transect area is accounted for to allow mass balance calculation of plume or system scale behavior.

\section{Monitored Natural Attenuation}

“"Monitored natural attenuation' ... refers to the reliance on natural attenuation processes (within the context of a carefully controlled and monitored clean-up approach) to achieve sitespecific remedial objectives within a time frame that is reasonable compared to that offered by other more active methods. The 'natural attenuation processes' that are at work in such a remediation approach include a variety of physical, chemical, or biological processes that, under favorable conditions, act without human intervention to reduce mass, toxicity, mobility, volume, or concentration of contaminants in soil or groundwater."

(EPA, 1999; pg. 3)

\section{Attenuation Capacity $\left(\mathbf{A}_{\mathbf{c}}\right)$}

The attenuation capacity is the general term that describes the amount of a contaminant that can be assimilated and attenuated within an identified subsurface system volume. It is the composite impact of the active attenuation processes (consistent with the general definition of capacity as "the ability to receive, hold, or absorb"). Attenuation capacity can be quantified in various ways and with a variety of units depending on the mathematical formulation used (this is analogous to the general term groundwater flow being described using a darcy velocity, a seepage (or pore) velocity or a discharge).

\section{Integrated Attenuation Rate (i $\left.\mathbf{A}_{\mathbf{r}}\right)$}

The integrated attenuation rate is a variable that can be used to quantify the Attenuation Capacity. The $\mathrm{iA}_{\mathrm{r}}$ is a mass balance based measure of the dynamic and sustainable contaminant destruction, delay, and dilution processes occurring within a system. The $\mathrm{iA}$ is the summation of all of the processes expressed as the total potential for attenuation occurring within a defined volume in units of contaminant mass attenuated per unit time (e.g., $\mathrm{Kg} / \mathrm{yr}$, $\mathrm{g} /$ day or the like). At steady-state, the attenuation capacity of a given part of a system is the difference between the upgradient integrated mass flux (i.e., contaminant loading) and 
downgradient integrated mass flux (contaminant discharge). The $\mathrm{iA}_{\mathrm{r}}$ accounts for flow

dynamics, the delivery rates of necessary reagents, and the conditional rates of the various processes within the volume.

\section{Sustainable enhancement}

A sustainable enhancement is an intervention action that continues until such time that the enhancement is no longer required to reduce contaminant concentrations or fluxes. 


\subsection{EXECUTIVE SUMMARY}

Monitored natural attenuation (MNA) and enhanced attenuation (EA) are two environmental management strategies that rely on a variety of attenuation processes to degrade or immobilize contaminants and are implemented at appropriate sites by demonstrating that contaminant plumes have low risk and are stable or shrinking. The concept of a mass balance between the loading and attenuation of contaminants in a groundwater system is a powerful framework for conceptualizing and documenting the relative stability of a contaminant plume. As a result, this concept has significant potential to support appropriate implementation of monitored natural attenuation (MNA) and enhanced attenuation (EA). For mass balance to be useful in engineering practice, however, it is necessary to quantify it in practical ways that facilitate overall site remediation and which are consistent with existing regulatory guidance.

Two divergent philosophies exist for quantifying plume stability - empirical and deterministic.

The first relies on historical contaminant concentration data and bulk geochemical information from a monitoring well network and documents plume stability using trend analysis and statistical tools. This empirical approach, when feasible, provides powerful and compelling documentation of plume behavior and mass balance. It provides an interpretation on a relevant scale under field conditions. It integrates the operative attenuation processes measured by observing their actual impact on the plume. The power of the empirical approach was recognized early in the development of MNA guidance and protocols and it is currently the basis of the three lines of evidence used in MNA studies. The empirical approach has some weaknesses, however. It requires a relatively long period of undisturbed historical data. Thus it cannot be effectively applied to sites where active remediation was initiated quickly and is currently operating. It cannot be used as a tool to determine how much source removal is needed or when to turn off active remediation and transition to MNA. It cannot be used to evaluate potential enhancement options (unless a long period of post enhancement monitoring is planned). It provides only indirect information about process and treats the plume as a "black box." The empirical approach has the advantage that, when sufficient monitoring data are available, the attenuation capacity can be defined inexpensively and with a high degree of certainty. 
Deterministic is defined as a process that assumes that "events proceed in a fixed predictable fashion." Models, ranging from simple to complex, are the primary tools for deterministic evaluation of plume stability and projection of plume behavior under changing conditions.
Alternatively, a deterministic approach can be used to assess mass balance and plume stability. In this approach, the physical, chemical, and biological attenuation processes are used to assess contaminant loading and attenuation. The deterministic approach has the advantage that, when sufficient hydrologic, geochemical, and microbiological data are available, it is possible to project how a system will respond to contaminant removal actions or enhancements of natural attenuation processes. The "black box" of the plume is taken apart, quantified, and put back together again. The disadvantage of the deterministic approach is that it is difficult to measure all or most of the relevant hydrologic, geochemical, and biological parameters with any certainty.

Case studies over the past decade demonstrate that empirical and deterministic approaches to MNA/EA are not mutually exclusive. These studies document that improved decision support and efficiency result by combining these methods based on the individual challenges presented by a given site. Whenever possible, the empirical approach is used to quantify mass loading and attenuation capacity (mass of contaminant/unit time) at particular sites. This is the most effective way to demonstrate the efficiency of ongoing natural attenuation processes in accordance with current regulatory guidance. But in addition, the monitoring well networks needed to apply the empirical approach can also yield estimates of the hydrologic, geochemical, and biological parameters needed to apply deterministic models. These models can then be used to estimate how contaminant behavior will change over time, as contaminant mass is removed, or if attenuation mechanisms are enhanced by engineering methods. The dual use of these empirical and deterministic approaches can help integrate the use of MNA and EA for overall site remediation.

Throughout this document, the examples and case studies selected address chlorinated organic compounds - these are the target compounds being addressed by the DOE MNA/EA Alternative Project. Focusing on this specific and challenging problem set will assist in the accelerated development of cost effective and environmentally protective technical solutions to address the real-world cVOC plumes at DOE sites, and other sites, throughout the nation. Except for a few cVOC specific transformation processes, the ideas underpinning the proposed mass balance concept are general and portable. They will support MNA/EA for other classes of contaminants, such as metals and radionuclides, and can be used to assist in source removal and enhancement decision-making. 


\subsection{INTRODUCTION}

Natural attenuation processes such as biodegradation, dispersion, dilution, sorption, chemical transformation, and volatilization affect the fate and transport of contaminants in all hydrologic systems. When these natural processes are shown to be capable of attaining site-specific remediation objectives in a time period that is reasonable compared to other alternatives, they may be used as a component of overall site remediation. This remedial strategy is known formally as Monitored Natural Attenuation (EPA, 1999) or MNA. In addition, these natural processes may be enhanced by various engineering methods in order to increase their overall effectiveness, a process known as enhanced attenuation (EA).

The most direct approach to demonstrating the effectiveness of natural or enhanced attenuation is to compare rates of contaminant loading to rates of contaminant attenuation in a given system. In classical waste-water engineering, this comparison is made by comparing contaminant loading to the assimilative capacity of a surface-water body (Chapra, 1996). By analogy, the efficiency of natural attenuation in groundwater systems can be assessed by comparing waste loading to the attenuation capacity (Chapelle and Bradley, 1998). Conceptually, it is clear that when contaminant loading is large relative to attenuation capacity, the environmental impacts will be similarly large. Conversely, when contaminant loading is small relative to the attenuation capacity the environmental impacts will be smaller. Thus, the key to assessing natural attenuation lies in comparing rates of mass loading to rates of natural attenuation. This comparison can be made quantitative using the principles of mass balance.

For example, the development of a contaminant plume (Figure 2-1) can be roughly described in four stages: expanding, stable, shrinking, and exhausted. The general requirements for environmental strategies that rely on attenuation are that the plume poses minimal risk (often by meeting a concentration standard at an agreed location) and that the plume will not expand. Thus, a key element in implementing MNA and EA is documenting that the plume is stable, shrinking, or exhausted. Plume stability can be conceptualized as a balance between the delivery to and removal of contaminants from any groundwater system.

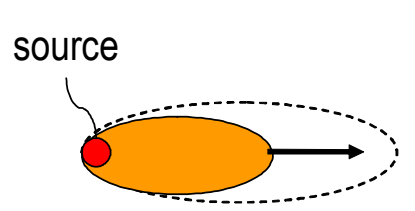

EXPANDING

I.

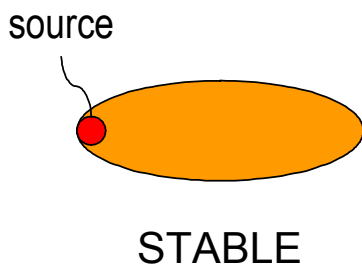

II.

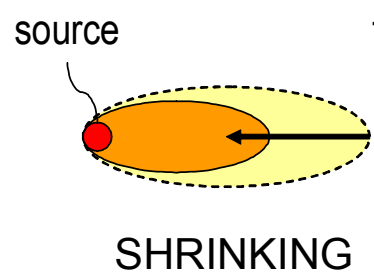

III. former source

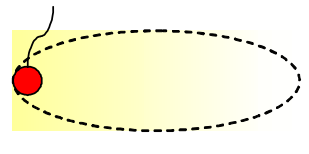

EXHAUSTED

IV.

time evolution of a plume if it undergoes attenuation

Figure 2-1. The four stages describing the development of a contaminant plume (adapted from Newell and Conner 1998) 
Mass balance is a simple accounting process that keeps track of loading (or inputs), accumulation, destruction/creation, and releases (or outputs). This is easily seen for a system in which water is the substance being accounted (Figure 2-2). As shown, at steady-state, the water entering from up-gradient plus the water entering as infiltration must equal, or balance, the water flowing out of the system. If infiltration is reduced, then the water flowing out of the system will decrease until a balance is re-established. Alternatively, if we started the system with no water in it, then the water entering the system would have to balance the water building up within the system plus the water flowing out. The simple accounting process allows all possible conditions to be evaluated.

Contaminants can be addressed in an analogous fashion with one significant difference various attenuation mechanisms can affect contaminants within the system. Some of these, such as biological and abiotic degradation, destroy contaminants. Some may generate or create contaminants (e.g., as daughter products from a degradation mechanism). Some, such as sorption or dispersion, may delay/immobilize contaminants or reduce concentrations. An example steady-state mass balance for contaminants is depicted in Figure 2-3.

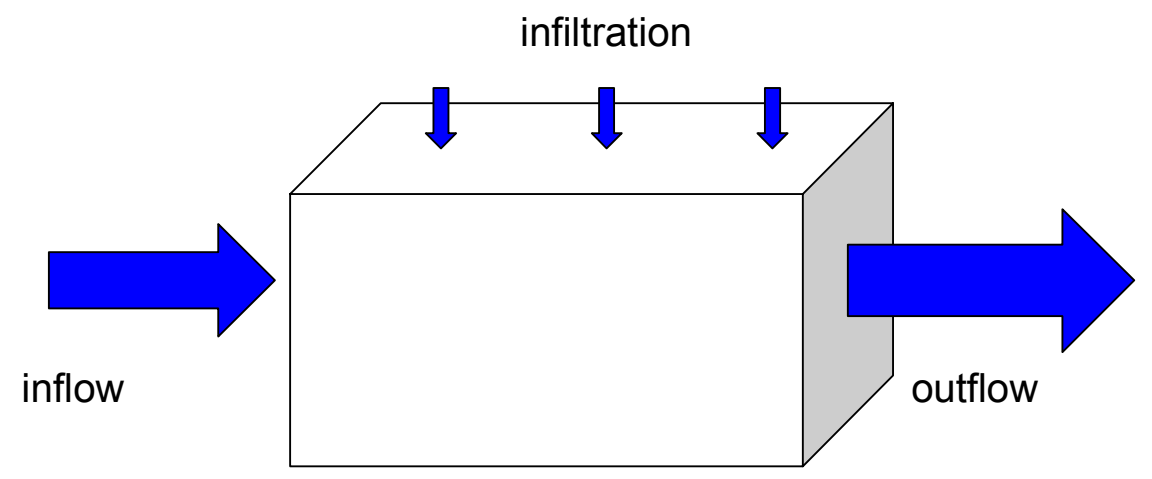

Figure 2-2. Steady-state mass balance example for water in a subsurface system

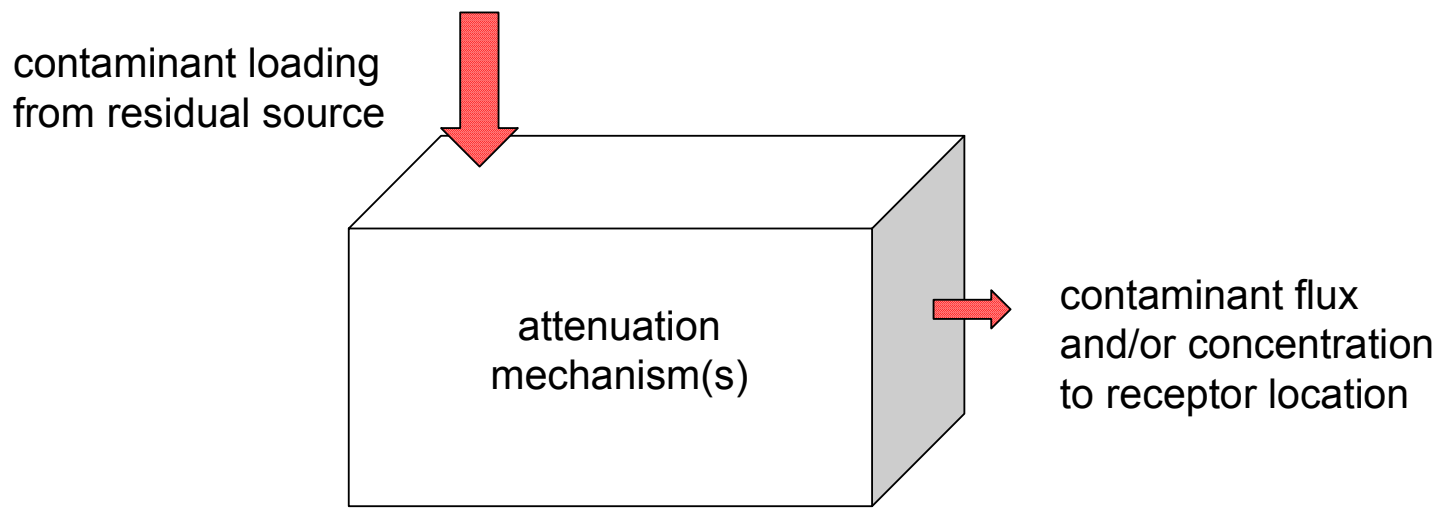

Figure 2-3. Steady-state mass balance example for contaminants in a subsurface system 
For a variety of situations, the general linkage between mass balance and plume stability is depicted in Figure 2-4. If attenuation mechanisms are weak or if the loading is high, then plumes will continue to expand. If natural attenuation mechanisms are moderate or high and/or loading is low, then plumes will tend to expand, stabilize, and then shrink depending on the precise balance. The current status for such a plume will be determined by where it is in the evolution process. The entire process, however, can be followed using mass balance accounting methods. The final panel in Figure 2-4 shows a plume where an appropriatesustainable enhancement has been implemented. In this case, the enhancement is designed to assure that a plume is in a stable or shrinking mode. An underlying feature of this approach is the inherent scalability of the mass balance approach.

The control box, or "volume," can be large (e.g., representing the plume or site) or it can represent a region or even a tiny portion of the plume (as is done in constructing numerical models). In every case, the mass balance is quantified based on the idea of an Integrated Mass Flux (iMF) - the iMF entering or leaving the overall system or at various transects or subzones within the system. The fundamental characteristics of the mass balance approach make it useful in linking empirical and deterministic methods.

As discussed in the following paragraphs, the mass balance can be quantified in a composite fashion using trends and statistics on the overall plume, or it can be evaluated by examining and cumulating the various processes within the system. In all cases, the first step in successfully applying a mass balance approach is developing a clear and agreed definition of the target plume volume and then determining the balance between contaminant loading and the attenuation capacity of that given vadose-groundwater system. Under this scenario, the feasibility of MNA is related to the contaminant loading combined with the composite "rate" of the relevant attenuation processes. Similarly, the overall timeframe of remediation is related to the source quantity combined with the composite "rate" of the relevant attenuation processes. If the attenuation capacity is too low for plume stability, options for enhancement can be examined.

There are many complexities in implementing a mass balance approach. For example: what is the best method to account for spatial and temporal variation in the attenuation capacities? What is the best approach to quantify these rates in a simple fashion for practical use at realworld sites? These topics are the basis of some of the key science and technology targets for advancing MNA and EA. A careful evaluation of mass balance may help in developing a framework to assist regulators and "corporate" managers develop a technical basis for determining the requirements for each source removal action and integrating MNA/EA into the sequence of remediation for each site. 


\section{Simplified Block Diagrams}
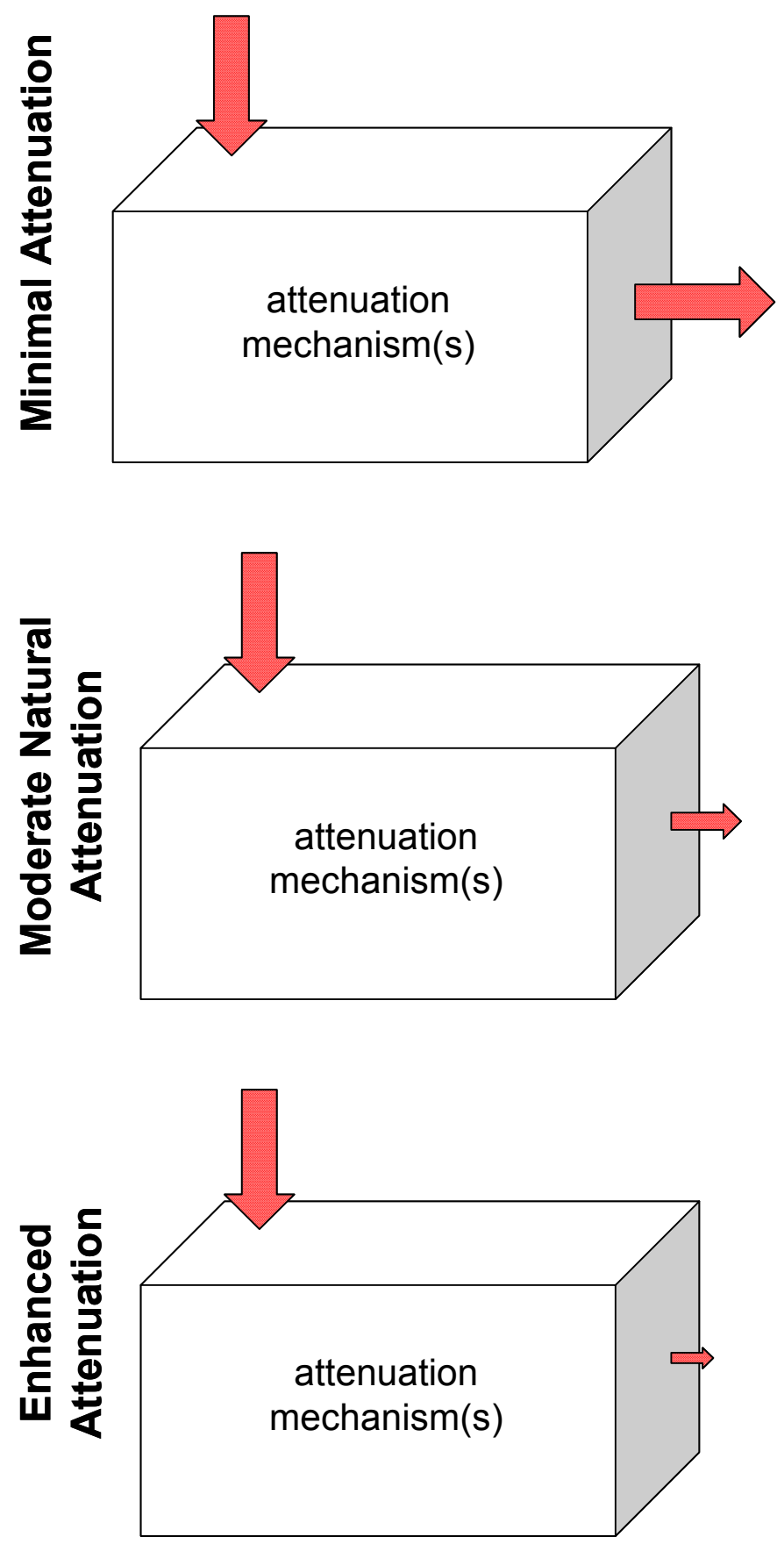

\author{
Probable \\ contaminant \\ plume status
}

Expanding
Expanding, Stable or Shrinking

Figure 2-4. Simplified steady-state contaminant mass balance block diagrams for a variety of scenarios

Stable or

Shrinking 
Linkage of remediation goals to concentration and mass balance is an important topic that has been the focus of scientists, engineers and policymakers. Traditionally, drinking water standards (which serve as a de facto remediation goal) are set by extrapolations from assumed average concentrations in water consumed over a lifetime. The assumptions apply to volumeweighted concentrations in drinking water consumed over many years. For consistency, such a standard should be applied to the average concentration in a volume of groundwater that would reasonably be extracted and used for drinking water supply. As a means to scale that reasonable volume, consider the volume of water that is produced and distributed by a household or a regulated water supplier using the necessary geometry and coverage of a water production well. Recent work by Einarson and Mackay (2001) used the integrated mass flux of a contaminant in a plume to calculate the concentration that would be produced by a water supply well, not a monitoring well. The analysis suggested that flux based measures may be a useful adjunct to traditional methods when developing environmental remediation goals. 
In implementing environmental regulations and policies, state regulatory agencies are typically on the front line. They are often the government interface to industry and site owners and are responsible for real-world implementation. The initial development and promulgation of environmental policies and regulations, while typically based on sound science and informed by broad comment, often leaves many unanswered details and may not prove to be robust in application when faced with the diversity of possible scenarios. To meet these challenges, state regulators have fostered organizations to allow communication of lessons learned and successful strategies (for example the Interstate Technology and Regulatory Council (ITRC), State Coalition for Remediation of Dry Cleaners (SCRD), and the like). They have also developed practical guidance documents and tools to help the site owners and their contractors do their work efficiently and in a manner that will meet the regulators expectations for content and quality. For MNA, in general (and for MNA of chlorinated solvents, in particular), there has been a substantive and innovative effort by the state regulatory community. The guidance and innovation result, in part, from the difficulty in efficiently assessing plume stability using the EPA protocol mandated "Multiple Lines of Evidence" in a disciplined fashion. How many wells are appropriate? How much data? Which parameters are most important in each state or region based on the local conditions? How does one organize the report? In a complex situation, such as most MNA decisions, there will always be uncertainty. Development and implementation of MNA guidelines was needed to provide tools for defining allowable uncertainty and resolving disputes.

This state-led front-line MNA development activity encouraged significant thought. Many of the current state guidance documents anticipate and presage the concepts being explored and expanded in the DOE MNA/EA Alternative Project. For example, Minnesota developed a guidance document in 1999 (only one year after the EPA protocol) that described a five-phase process for implementing MNA including 1) screening for natural attenuation, 2) detailed site characterization and refinement of conceptual models, 3) rate analysis of site-specific attenuation kinetics, 4) modeling - simulating natural attenuation, and 5) implementation and long-term monitoring. This sequence anticipates the DOE recommendation of considering a combined empirical and deterministic approach to MNA/EA and the recommendation of performing characterization and monitoring using the following sequence: screening characterization, decision characterization, process monitoring, and system performance monitoring. Minnesota provides recommendations about how to interpret the many parameters listed in the EPA protocol and gives recommendations on practical ways to use a simplified geochemical score sheet for multiple contaminants and for other mechanisms besides reductive dechlorination. Further, this guidance, as well as recent guidance from the state of Washington (WA-DE, 2004), encourage the consideration of MNA as a mass balance. Washington provides examples of calculating integrated mass flux across the plume and specifically describes MNA as follows: "the ability of natural attenuation to reduce contaminant concentrations depends on whether the natural attenuation rate in the plume exceeds the mass loading rate from the source." Wisconsin provides similar advancements and discusses in detail a variety of attenuation mechanisms and their potential for contribution to MNA. All of the guidance documents provide specific guidance on the type and amount of data to collect. They provide handy flowcharts and data collection sheets. Many of the states have developed companion spreadsheets to facilitate the required data analysis (e.g., the Mann Kendall test). This practical and forward thinking effort represents the true condition for MNA implementation in the country and should be the base upon which future advancements are made.
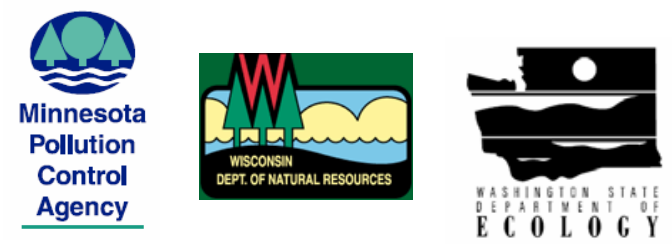


\subsection{PROCESSES CONTRIBUTING TO ATTENUATION FOR CHLORINATED SOLVENTS}

According to EPA (1999):

The term "monitored natural attenuation," as used in this Directive, refers to the reliance on natural attenuation processes (within the context of a carefully controlled and monitored clean-up approach) to achieve site-specific remedial objectives within a time frame that is reasonable compared to other methods. The "natural attenuation processes" that are at work in such a remediation approach include a variety of physical, chemical, or biological processes that, under favorable conditions, act without human intervention to reduce the mass, toxicity, mobility, volume, or concentration of contaminants in soil and ground water. These in-situ processes include, biodegradation, dispersion, dilution, sorption, volatilization, and chemical or biological stabilization, transformation, or destruction of contaminants. Monitored natural attenuation is appropriate as a remedial approach only when it can be demonstrated capable of achieving a site's remedial objectives within a time frame that is reasonable compared to that offered by other methods and where it meets the applicable remedy selection program for a particular OSWER program. EPA, therefore, expects that monitored natural attenution typically will be used in conjunction with active remediation measures (e.g., source control), or as a follow-up to active remediation measures that have already been implemented.

Along with large-scale forces such as groundwater flow rate, infiltration, and geology, the processes listed above influence plume behavior within the agreed system boundaries and determine the amount of plume expansion and ultimately control the plume
See Case Study 3-1. In this case study, protocols developed by Minnesota, Wisconsin, and Washington are examined to see how they anticipate current technical developments in MNA science. size, stability and lifetime. Notably, some of these listed processes destroy contaminants biodegradation and chemical or biological transformation or destruction. Some of the processes physically delay, remove or immobilize contaminants - sorption, volatilization, and chemical or biological stabilization. And some simply reduce concentration by physical mixing-dispersion and dilution. Notably, the regulatory community prefers destructive processes to immobilization processes and prefers immobilization processes to mixing processes (e.g., MN-PCA, 1999; WI-DNR, 2003; WA-DE, 2004). Moreover, based on the recommended parameters measured within the EPA $(1998,1999)$ guidance, the primary acceptable destruction mechanism is biological reductive dechlorination. Recent data (e.g., Ferrey et al., 2004, Bradley, 2003; Butler and Hayes, 2001), however, indicate that other processes may be substantively contributing to chlorinated solvent attenuation at some sites providing a niche to incorporate research into future guidance. 
The weakest attenuation conditions are those in which contaminants are only impacted by mixing processes, such as dispersion. In this case, inputs from any significant steady-state source would lead to a long period of plume expansion and a large resulting plume. It is significant that a recent study of concentration trends beneath source areas (Newell and Adamson, 2005) suggests that the actual/observed releases from sources tend to decrease significantly over time rather than behaving in a steady-state fashion. Within a mass balance context, this observed real-world behavior suggests that immobilization processes, and even mixing processes, may provide a more significant role than previously recognized.

Importantly, attenuation processes are active in all systems and the composite impact of these attenuation processes is the attenuation capacity (consistent with the general definition of capacity as "the ability to receive, hold, or absorb"). The attenuation capacity can be quantified in various ways. For example, Chapelle and Bradley (1998) define an attenuation factor that captures the summation of the various attenuation processes in terms of concentration decay over a flow distance. The processes could also be quantified as a local attenuation rate $\left(\mathrm{A}_{\mathrm{r}}\right)$ in mass attenuated per unit volume per time $\left(e . g ., \mathrm{Kg} / \mathrm{m}^{3} / \mathrm{yr}\right)$. Alternatively, to explicitly support a mass balance, attenuation capacity can be quantified as an integrated attenuation rate $\left(\mathrm{iA}_{\mathrm{r}}\right)$. The $\mathrm{iA}_{\mathrm{r}}$ is the sum of the contaminant destruction, delay, and dilution processes occurring within the system at a given time. The $\mathrm{iA}_{\mathrm{r}}$ expresses the total potential for attenuation occurring within the defined volume in units of contaminant mass attenuated per unit time (e.g., $\mathrm{Kg} / \mathrm{yr}, \mathrm{g} / \mathrm{day}$ or the like). The $\mathrm{iA}_{\mathrm{r}}$ represents the cumulative contributions of all of the local attenuation rates within a plume volume and accounts for overall flow dynamics, the delivery rates of necessary reagents, and the conditional rates of the various destruction and sorption processes. It is important to note that the general concept of attenuation capacity can be supported by various model formulations and can be represented in a variety of variables. This is analogous to the general concept of groundwater flow which can be alternatively quantified in terms of pore velocity $(e . g ., \mathrm{m} /$ day $)$, darcy velocity $\left(e . g ., \mathrm{m}^{3} / \mathrm{m}^{2} /\right.$ day), or discharge (e.g., $\mathrm{m}^{3} /$ day). Just as with flow, attenuation processes will vary from place to place and vary over time and the modeling approaches need to be selected and matched to the site-specific needs. Throughout this report, we will use the term attenuation capacity as a general concept and will use a mass balance based formulation with the variable $\mathrm{iA}_{\mathrm{r}}$ to quantify this concept.

Figure 3-1 depicts the various processes contributing to attenuation of contaminants in the form of a graphical mass balance. In this figure, the width of the arrow represents the flux as the contaminant plume moves through the environment. At the source, the flux (arrow width) is the greatest. As the contaminant migrates, the various attenuation mechanisms reduce the central plume flux in proportion to the strength of each particular attenuation mechanism in the site-specific conditions. The width of the attenuation arrows is subtracted from the width of the plume migration arrow, impacting the amount of plume discharge and the overall growth and stabilization of the plume. In some cases, the attenuation is sufficient to serve as a reliable remedial option to achieve remediation goals. In other cases, the attenuation mechanisms are insufficient and additional actions are needed. These actions include source removal/destruction and active remediation, but as shown in Figure 3-2, enhancements to the natural system may be a viable and attractive option. Enhanced attenuation uses technology to 
sustainably alter the mass balance to achieve remedial goals. This is depicted by increasing the size of the attenuation arrows, concordantly decreasing the plume flux and growth.

Approaches to quantify the various processes and the various methods to reassemble the resulting pieces into a mass balance are addressed in the following sections.

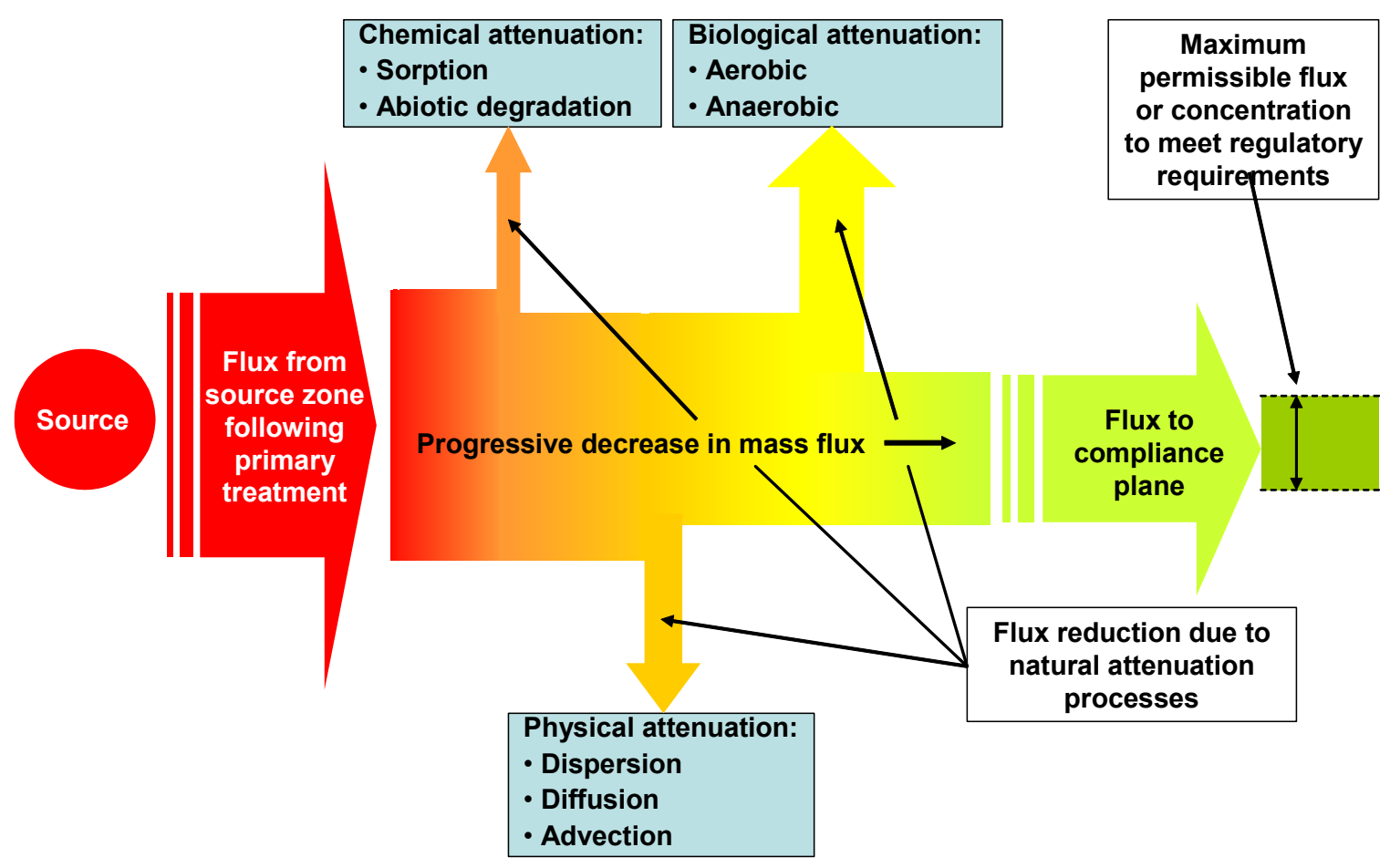

Figure 3-1. Graphical depiction of natural attenuation mass balance 


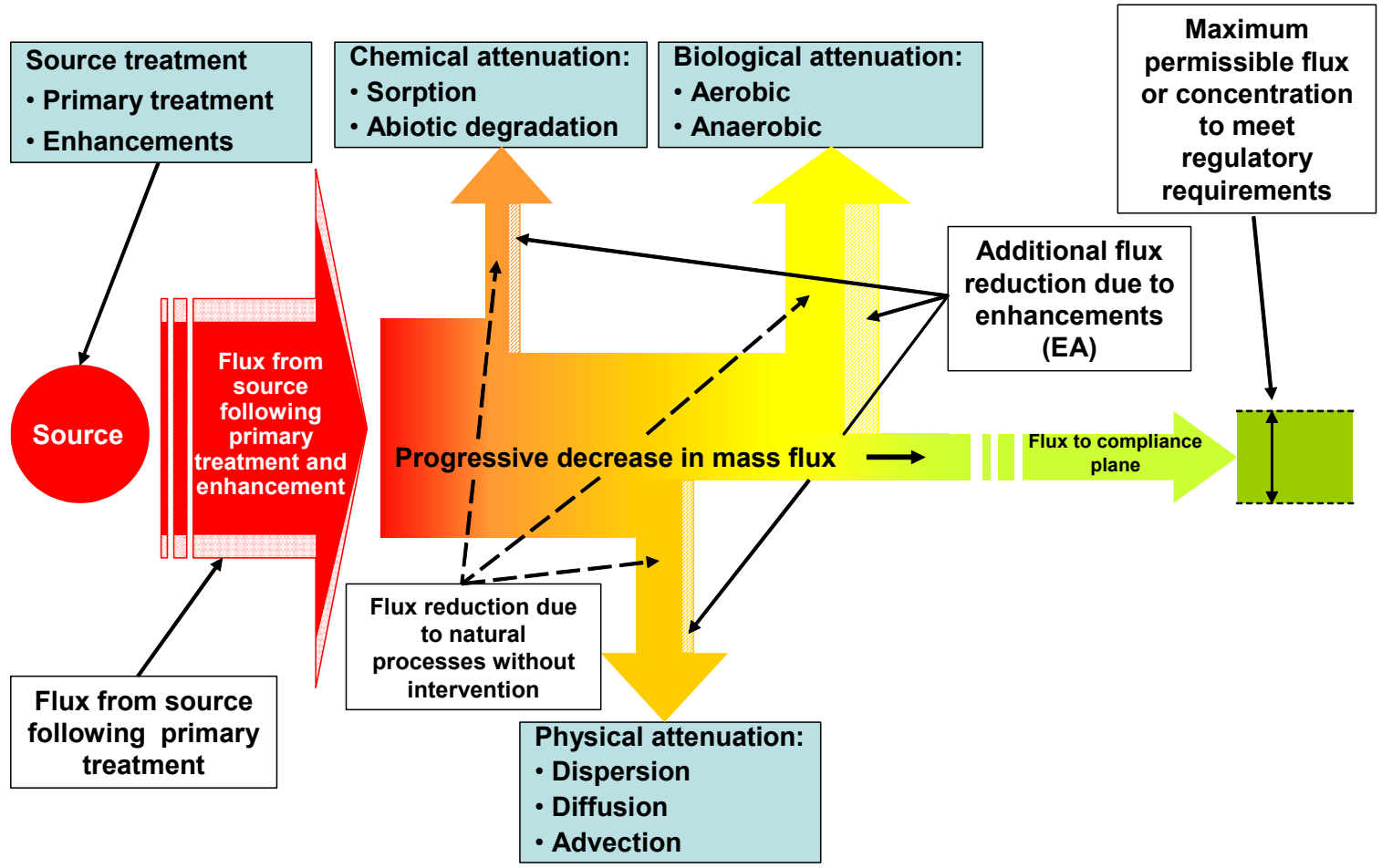

Figure 3-2. Graphical depiction of enhanced attenuation mass balance 


\subsection{ALTERNATIVE APPROACHES TO QUANTIFY MASS BALANCE}

An empirical approach to describing the mass balance of MNA is based on site-specific monitoring data and, under many conditions, provides the most accurate representation of the past and present behavior of the hydrologic system in question. The empirical approach to mass balance uses statistical measures of trends, geochemical conditions and measured concentrations and/or fluxes of contaminants moving through different transects of the hydrologic system. In some cases, fluxes can be estimated directly using properly designed monitoring well networks (Figure 4-1). In this formulation, the attenuation capacity of a given part of the system can be defined as the difference between the totalized or integrated mass flux across upgradient (e.g., $\left.\mathrm{iMF}_{1}\right)$ and downgradient (e.g., $\left.\mathrm{iMF}_{2}\right)$ transects, and can be expressed in units of contaminant mass attenuated per unit time (e.g., $\mathrm{Kg} / \mathrm{yr}$, g/day or the like). This method requires that the leading edge of the plume be beyond the most downgradient transect being tested.

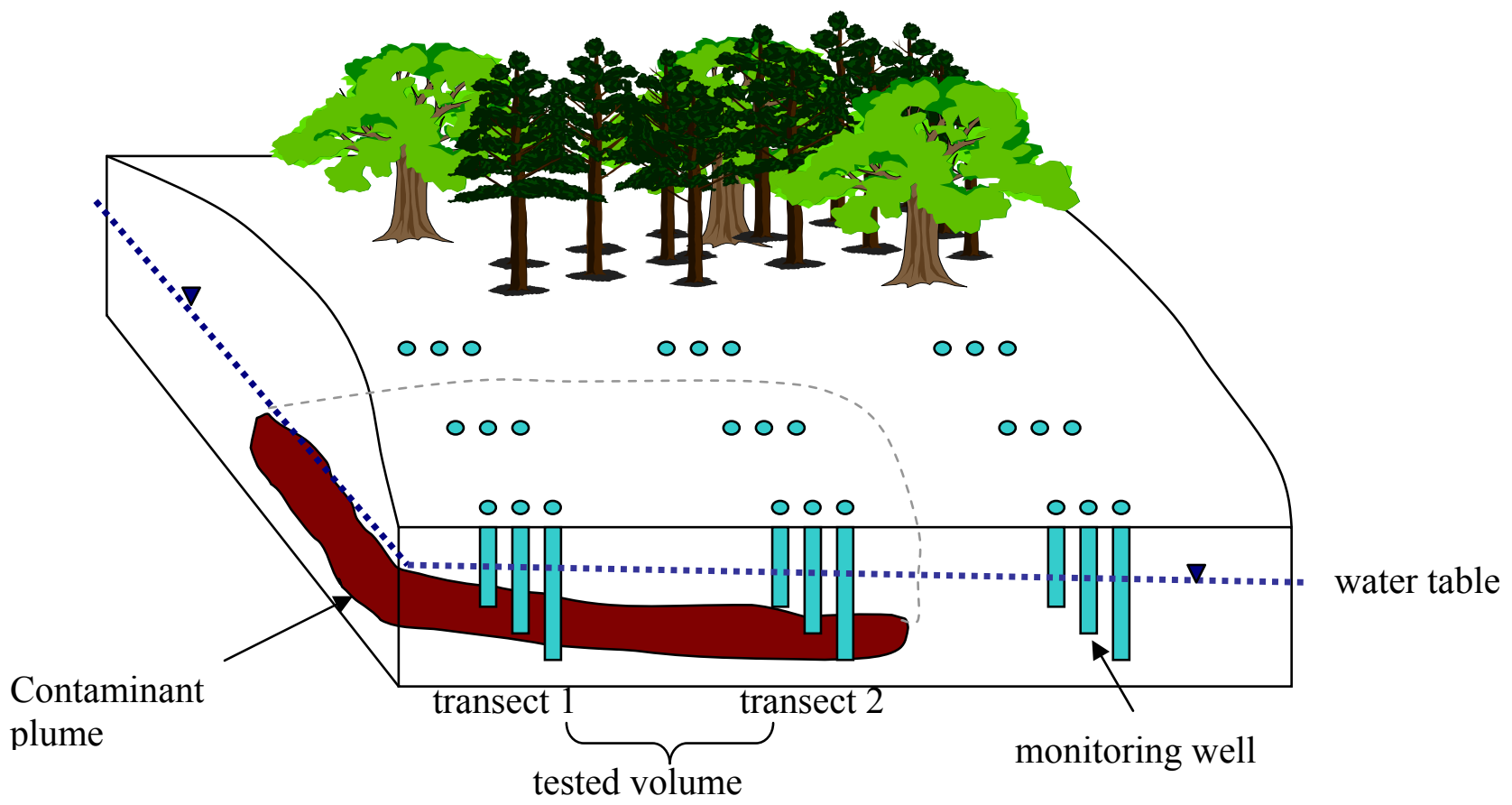

Figure 4-1. The Empirical Approach to Describing the Mass Balance of a System Depends on Site-Specific Monitoring Data (adapted from Adams et al. 2004) 
The chief limitation of the empirical approach is that it is not suitable for predicting the future behavior of the hydrologic system. It is observed, for example, that non-aqueous phase liquid (NAPL) dissolution rates change over time, changing the contaminant load being delivered to the systems. Also, the availability of electron donors and acceptors that drive ambient biodegradation processes may also change over time. For these reasons, the empirical approach is inherently limited in its ability to project forward and predict system behavior in the future. Furthermore, such future projections of the efficiency of MNA are often critical in selecting appropriate remediation strategies, in assessing sustainability, and in estimating time of remediation.

See Case Study 4-2. In this case study, Semprini and others document an early application of the use of transects to develop a mass balance and interpret the significance of the results.

\subsection{DEVELOPING MASS BALANCE EQUATIONS}

In addition to the empirical determination of mass balance, it is also possible to describe the mass balance mathematically in terms of the processes that control transport and transformation. This approach assumes the dominant processes are known and these can be formulated into equations describing transport. Using estimates of key parameters for the field site, the resulting set of equations can be solved using a variety of techniques.

Consider a control volume of dimensions $\Delta x, \Delta y, \Delta z$. Groundwater, with a darcy velocity of $\mathrm{v}_{\mathrm{d}}$ flows in the $\mathrm{x}$-direction as shown:

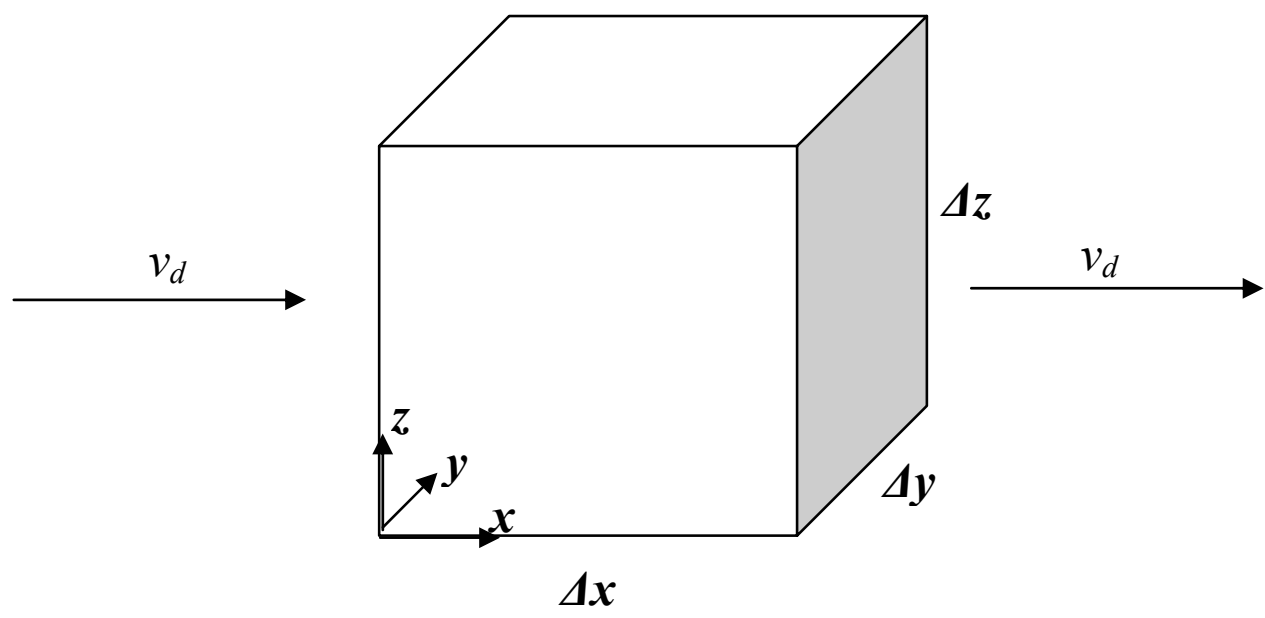

Figure 4-2. Typical "control volume" used to develop mass balance equations for contaminant plumes 
Wiedemeier et al. (1999), Suarez and Rafai (2002) and others suggest that alternative interpretation approaches applied to traditional field measurements may be a powerful and appropriate tool for assessing integrated mass fluxes within a plume. These fluxes, in turn, can be used to calculate degradation rates and mass balances. Their recommendations are already incorporated into various MNA related training classes and guidance documents and the concepts set the groundwork for a mass balance view of plume stability and for using field data as a key element in quantifying the mass balance. The DOE MNA/EA project is examining the potential to build on these concepts using alternative well geometries (e.g. horizontal wells or trenches) for sampling, new field methods (such as the push-pull test), and new sensor technologies. Other potential advancements include accounting for electron donors and acceptors in the mass balance as well as the contaminants. Electron acceptors and donors can be evaluated using the same types of traditional field data. Box 1 is an example of the use of a field/transect-based measurement of integrated mass flux (excerpted from WI-DNR, 2003).

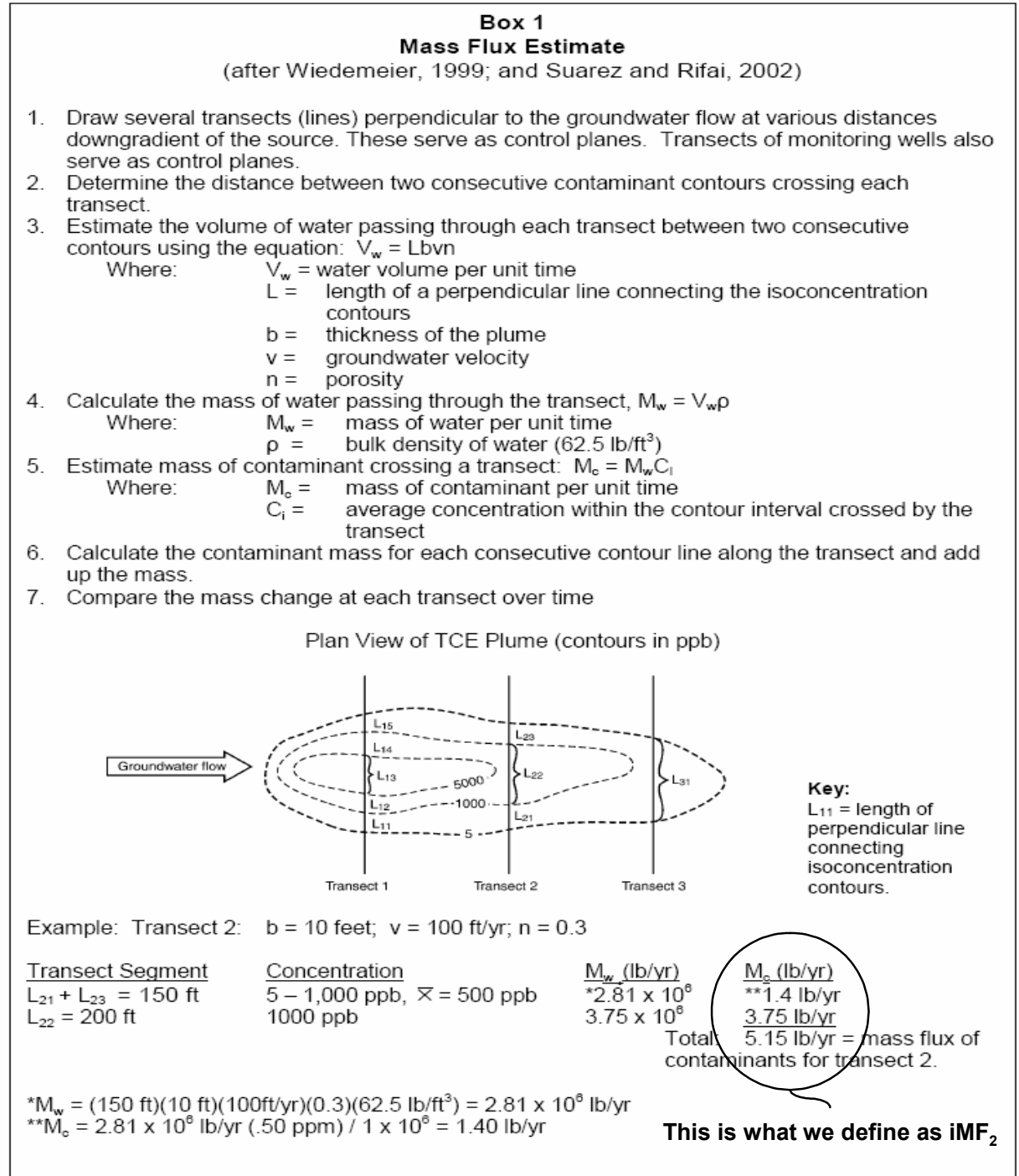


In developing the concepts of MNA, many investigators have studied plume dynamics and stability using concentration mapping, geochemical indicators, shifts in the ratio of parent to daughter compounds over time and space, and/or fluxes at various transects in a quasi-steadystate plume. Semprini et al. (1995) provide an early exemplar of this important type of case study. In this study, approximately 155 samples were collected in a "longitudinal" transect and two "transverse" transects - the transverse transects intercepted the migrating contaminant plume at two flow distances. Samples were collected in a grid pattern within these transects using closely spaced boreholes and depth discrete sampling at 5 foot intervals within the aquifer. The contaminant concentrations, geochemistry, and parent daughter ratios indicated significant reductive dechlorination. Importantly, the data when presented as two-dimensional transects showed concordance between the major contaminant transformation zones and the areas of high methane and low sulfate. Further, these transects provide the basis for estimating flux for the two transverse transect distances. In this case, the estimates of mass and mole fluxes of the chlorinated hydrocarbons, ethene, and methane were made based on the mean aqueous concentration, the Darcy flow velocity (perpendicular to flow), and the cross sectional area represented by the sampled concentration. The total molar flux of chlorinated hydrocarbons plus ethene at the upgradient transect was about 2 times greater than the downgradient transect. Semprini et al. (1995) noted that if the "transport conditions were steady-state, then the total mole flux into the upgradient transect should equal the mole flux out of the downgradient transect," and that "a greater mole flux upgradient means that accumulation (increase in storage) in total chlorinated hydrocarbons and ethane occurs between the two transects." The greatest mole flux in both transects was represented by DCE, followed by TCE indicating that significant anaerobic transformations are taking place. Ethene

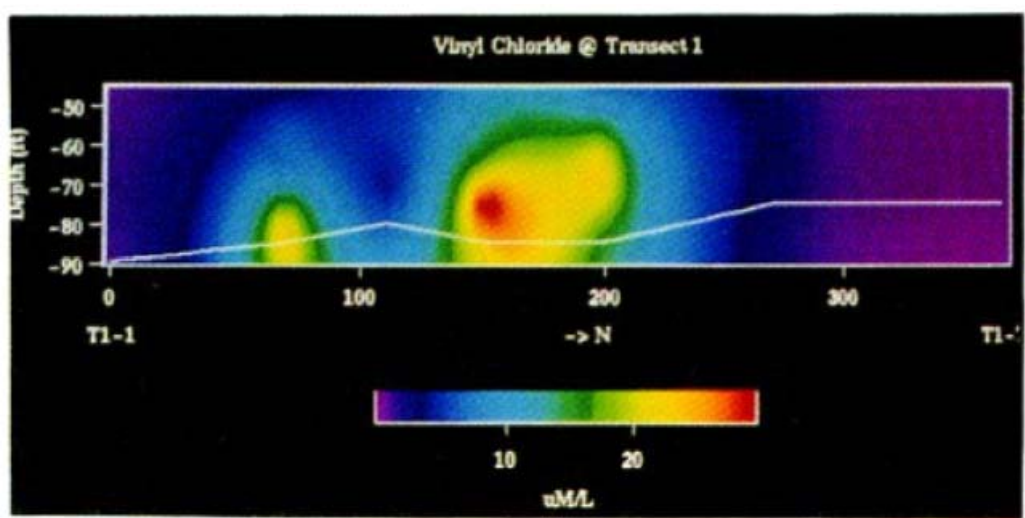

Example of transect data. This image shows vinyl chloride; other data from the transects included the parent contaminant (TCE), related daughter/end products, and key geochemical indicators represented $8-22 \%$ of

the total mole flux indicating a significant fraction of the chlorinated hydrocarbons are being completely dehalogenated. This was an important early case study because it pointed out the importance of considering if the plume was at steady-state. This study indicated that a flux based monitoring concept may provide interesting and unique value. Later investigations have refined these ideas. For example, such studies indicate that flux estimation processes may be improved by weighting point concentration data using local hydraulic conductivity (instead of weighting concentrations by a representative surface area assigned by equally weighted distances (i.e., Theissen Polygons) and using average Darcy velocity). Flux samplers that use contaminant sorption and simultaneous tracer leaching have been developed for deployment in wells to directly estimate local fluxes. An alternative that has had limited study, but may have practical utility, is the use of long well screens that provide a composite sample from the thickness of an aquifer in rough proportion to the concentrations and hydraulic conductivities. These topics are being extensively studied by the EPA, Department of Defense (DOD) and DOE and new tools and techniques are anticipated. 
The transport equation is based on conservation of (chemical) mass. For any control volume, this law can be stated as:

Rate of mass accumulation inside volume $=$ Rate of mass entering volume - Rate of mass leaving volume - Rate of mass destruction inside volume

Each of these terms can be developed from basic principals. The resulting mathematical relationships, in concert with assumptions about boundary conditions (e.g., assumed source release profile with or without removal actions) and solution techniques, have been used to generate many different types of deterministic models that apply to a variety of conditions.

\section{See Appendix A for an example derivation of a deterministic model from a mass balance applied to the control volume depicted above.}

In many groundwater systems, the most important processes affecting the transport of chlorinated ethenes are advection, hydrodynamic dispersion, sorption, and biodegradation. These processes, in combination with an understanding of the time varying inputs from a source zone, control the development, stabilization, potential discharge, and ultimate shrinkage of a groundwater plume. Because deterministic models attempt to identify and quantify the important attenuation processes and they project plume behavior and response to changes in source inputs (loading), they are useful adjuncts to field based determinations of stability.

Examination of the types of calculations incorporated into estimating loading from a source provides insight into the potential value of formulating the problem of MNA/EA as a deterministic model. The dissolution of NAPL mass and release from the source represents the loading to a contaminated system. For a single component source, this release is often related to the contaminant solubility, and for a multi-component source, the release is often related to the effective solubility (the solubility of each solute multiplied by its mole fraction in the NAPL analogous to Raoult's law). Other factors that impact the loading include the dynamics of flow through (or infiltration through) the source zone, diffusion or dispersion from the sources in low permeability materials, volatilization of the source, and the like. Similar to degradation, many models have been developed to describe the source release and plume loading and these vary widely in their predictions related to a variety of pragmatic questions. Does removal of $90 \%$ of the mass using source treatment reduce the contaminant release by $90 \%$ ? How much source treatment is needed to stabilize a plume? Many models assume simple source functions - the most conservative "worst-case" assumption is a constant and inexhaustible release. Other common assumptions are an exponentially decaying release, or a release rate that is proportional (in some fashion) with remaining mass. In most of these models the source term is in units of mass per time.

Recent papers (e.g., Falta et al., 2005a and 2005b) review the derivations of various deterministic models for chlorinated organics and discuss the important differences between the key assumptions and solution techniques. These valuable papers also examine plume stability, the impact of partial source removal on contaminant plume behavior, time to reach remediation goals, and risk. 


\subsection{SUMMING THE PROCESSES CONTRIBUTING TO NATURAL ATTENUATION}

Many processes that contribute to natural attenuation, including hydrodynamic dispersion, advection, sorption, and biodegradation, and NAPL dissolution, when formulated in consistent units can be summed to give overall concentration changes of a solute $\mathrm{C}$ with time $(\partial \mathrm{C} / \partial \mathrm{t})$. Thus, $\partial \mathrm{C} / \partial \mathrm{t}$ throughout the target domain is set equal to the sum of advection carrying contaminants in and out, hydrodynamic dispersion in and out, sorption, and biodegradation. Other processes that can be included as needed include inputs from internal sources or degradation of parent compounds, abiotic degradation (e.g., hydrolysis or interaction with minerals in the sediment), cometabolic degradation, competing reactions and nutrient limitations, etc. A typical governing equation for solute transport is:

$$
R \frac{\partial C}{\partial t}=-v_{s} \frac{\partial C}{\partial x}+D_{x} \frac{\partial^{2} C}{\partial x^{2}}+D_{y} \frac{\partial^{2} C}{\partial y^{2}}+D_{z} \frac{\partial^{2} C}{\partial z^{2}}-\lambda C \ldots
$$

Where the variables are standard as defined in Appendix A and in the list of symbols.

This summary equation can be related back to the mass balance by assuming that the concentration changes are occurring to or within a specific volume $(\mathrm{V})$. This is the same conceptual volume that was introduced earlier. Most models require that this volume be relatively small to meet mathematical requirements and to provide accurate results. Nonetheless, in a general sense, $\mathrm{V}$ can be a small volume (such as a model element), a subregion within a plume (such as the zone between two transects), or an entire site.
An equation that that calculates the change of concentration versus time in a control volume is often called a "transport equation," but what it also represents is a mass balance equation.

Note that this result is analogous to the empirical process presented earlier in Case Study 4.1 (Wiedemeier (1999), Suarez and Rafai (2002), and others). The attenuation capacity in the control volume is represented by destructive processes in this equation combined through a complex interaction between the dispersive and diluting processes and the concentration goals for the site. For simplicity, these processes can be considered as a lumped-composite rate, the integrated attenuation rate $\left(\mathrm{iA}_{\mathrm{r}}\right.$, mass/time). This rate typically varies in time and space but can be spatially or temporally integrated as needed to allow construction of a range of models as well as to serve as the basis for empirical estimation techniques.

\subsection{TIME OF REMEDIATION}

One important way that the deterministic mass balance approach can be used has to do with what has been termed the time of remediation associated with monitored natural attenuation. According to EPA guidance, MNA is appropriate to use as a remedial strategy when "...it will meet site remediation objectives within a timeframe that is reasonable compared to that offered by other methods" (EPA, 1999). Thus, estimating the amount of time required for natural or enhanced attenuation processes to attain regulatory objectives is a necessary step when evaluating MNA as a remedial alternative. 
In concept, estimating the length of time required for natural processes to remove a particular contaminant from a groundwater system is also a simple mass balance problem. If the initial mass of contaminant, $\mathrm{M}_{\mathrm{o}}$, present in the source and groundwater of a contaminated system is known, and if the rate that the contaminant is transformed or destroyed by attenuation processes, $\mathrm{iA}_{\mathrm{r}}$, is known, a mass balance equation can be written

$$
\left[\mathrm{M}_{\mathrm{o}}-\left(\mathrm{iA}_{\mathrm{r}} * \mathrm{t}\right)\right]=\mathrm{M}_{\text {remaining }}
$$

in which $t$ is time and $\mathrm{M}_{\text {remaining }}$ is the contaminant mass remaining in the system at any time $t$. Note that this highly simplified equation assumes a constant value for $\mathrm{iA}_{\mathrm{r}}$. The calculation can be made as site specific and complex as desired by allowing $i \mathrm{~A}_{\mathrm{r}}$ to vary in time and space and then integrating the result. The time of remediation can be defined as the time required to lower contaminant mass below a given threshold $\left(\mathrm{M}_{\text {threshold }}\right)$ :

or:

$$
\left[\mathrm{M}_{\mathrm{o}}-(\mathrm{iA} \cdot \mathrm{t})\right]=\mathrm{M}_{\text {threshold }}
$$

$$
\mathrm{t}_{\text {remediation }}=\left[\mathrm{M}_{\mathrm{o}}-\mathrm{M}_{\text {threshold }}\right] / \mathrm{i} \mathrm{A}_{\mathrm{r}}
$$

where the time of remediation for this simplified case is defined explicitly as given in equation (2), and refers to the length of time needed for a given mass of initial contaminant $\left(\mathrm{M}_{\mathrm{o}}\right)$ to be lowered below a given threshold $\left(\mathrm{M}_{\text {threshold }}\right)$, by the rate of attenuation processes $\left(\mathrm{iA}_{\mathrm{r}}\right)$ occurring in a groundwater system.

In addition to providing a working definition of time of remediation, equation 2 also indicates the kinds of information necessary to make such estimates. This information includes an estimate of the mass of contaminant present, and an estimate of the rate of ongoing natural attenuation processes acting on the contaminant. The principal technical problem, therefore, is to obtain reliable estimates of these variables. Clearly, the reliability of any remediation time estimates will be directly linked to the reliability of these estimates. In addition, equation 2 shows that determining remediation times requires the definition of an acceptable contaminant mass threshold. This threshold must be predetermined in order to make remediation time estimates.

In practice, however, the problem is much more complex then indicated by the equations presented above. The rate of natural attenuation processes $\left(i A_{r}\right)$, particularly rates of biodegradation, may vary with time, so that the rate that contaminants are destroyed may not be constant, but rather vary as a function of time and/or space (iA $=f(\mathrm{t}, \mathrm{x}, \mathrm{y}, \mathrm{z})$ ). Furthermore, many instances of groundwater contamination by chlorinated solvents can be traced to the release of NAPLs that dissolve over time at varying rates. Initially, contaminant plumes tend to grow and expand over time as relatively fresh NAPLs dissolve. At a certain point, however, rates of NAPL dissolution tend to come into equilibrium with the rate of contaminant removal by natural attenuation processes, and the plume comes into a quasi steady-state configuration. If the NAPL source continues to dissolve over time until it is exhausted, the contaminant plume will also shrink. Significant progress is being made on this topic, however. For example, Falta et al. (2005b) develop rigorous equations to examine these topics with several 
projections of the time course of the mass change in parent and daughter compounds in plumes for cases with and without partial DNAPL source remediation under a variety of conditions.

Clearly, estimating the time of remediation associated with given sites is a challenging problem. Nevertheless, this problem can be addressed by combining empirical and deterministic approaches to mass balances associated with MNA - and by developing a range of well documented tools to perform the analyses for sites of varying complexity. 


\subsection{INTEGRATING THE EMPIRICAL AND DETERMINISTIC APPROACHES TO MNA}

While fundamentally different, the empirical and deterministic approaches to describing and evaluating mass balance associated with MNA are not mutually exclusive. In fact, if applied systematically, these approaches can be highly synergistic. The empirical approach, for example, is entirely based on site-specific monitoring data and provides a mass balance that describes only that site at only that point in time. As such, it is capable of giving the most accurate description of past and present conditions providing that sufficient data have been collected. The deterministic approach, on the other hand, is based on universal, quantifiable physical and chemical processes that apply to all sites. Furthermore, the mass balance equations developed are time dependent. This, in turn, raises the possibility that the deterministic approach can provide estimates of the future behavior of sites. The accuracy of these predictions, however, depends on how well the time-dependent mass balance equations are constrained by actual site data. As such, the deterministic approach is capable of providing the most accurate description of future conditions providing that sufficient data have been collected. In other words, the empirical and deterministic approaches can be used in concert to most accurately describe the past and present behavior of a given site, as well as providing projections of future behavior.

One scenario of how the empirical and deterministic approaches can be used in concert to assess the ability of MNA to attain site-specific remediation goals is shown in Figure 5-1. It begins with an entirely empirical description of the system, with the attenuation capacity being estimated from monitoring data. Based on this data, a determination of whether MNA is by itself sufficient to attain site-specific remediation goals can be made. In practice, this usually consists of demonstrating that the plume is stable or shrinking, and that it is not impacting sensitive receptors. In cases where MNA is not by itself sufficient, at least two approaches can then be taken (Figure 5-1). On one hand, removal or treatment of highly contaminated source areas can be undertaken in order to lower the delivery of contaminant mass to the system. Alternatively, the attenuation capacity of the aquifer can be enhanced using engineered methods such as biostimulation, bioaugmentation, or phytoremediation. In either case, now that the system has been perturbed, the empirical approach is no longer sufficient to assess site behavior. To assess how the newly engineered system will behave over time, in particular estimating when remediation goals may be reached, requires the deterministic approach.

One of the useful features of an empirical description of a system is that data are available for "harvesting" model variables such as $\mathrm{D}, \mathrm{v}, \mathrm{K}_{\mathrm{d}}, \lambda$ from site data. Once these variables have been estimated, it is possible to construct a site-specific deterministic model (Figure 5-1). These models, in turn, can be used to estimate the future behavior of the site. In particular, it is possible to project into the future to estimate when site-specific remedial goals may be reached. While such projections are estimates, constrained entirely by the accuracy of the assumed model variables, they can be useful in comparing times of remediation associated with different engineering approaches. 


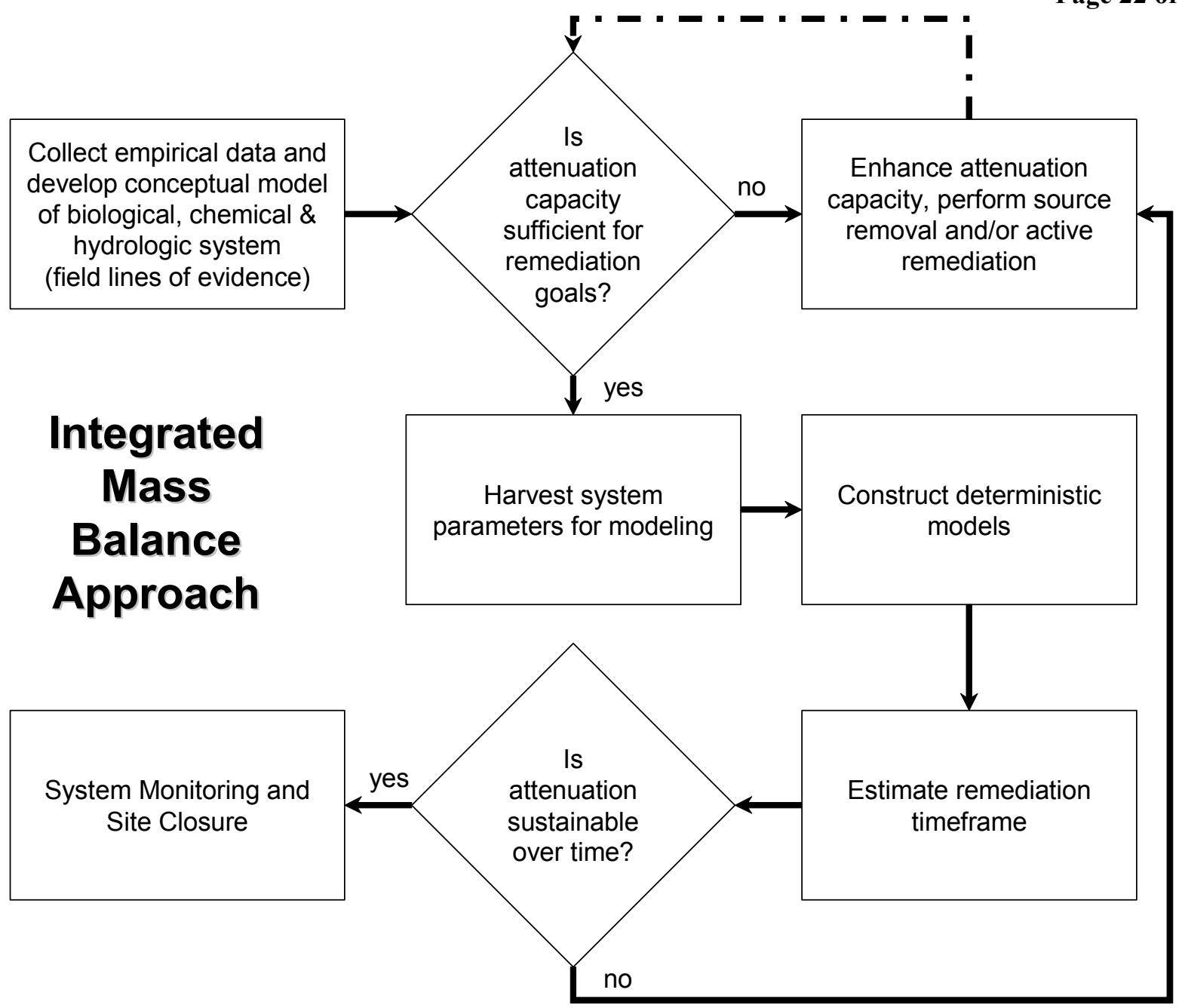

Figure 5-1. A Process for Integrating the Empirical and Deterministic Approaches for Calculating a Mass Balance

If the deterministic models indicate remedial goals may not be reached, it is possible to cycle back to the source removal/enhance attenuation capacity step and make appropriate adjustments. Several difficult problems remain, however. For example, if the attenuation capacity is not sufficient (Figure 5-1, highlighted line): How much source removal, enhancement and/or active remediation will be needed? How can attenuation capacity be estimated to allow turn-off of active remediation, when several years of empirical data without the system operating and with multiple lines of evidence are typically "required?" Here too, the mass balance concept may be helpful to develop a defensible and appropriate final flowchart with additional deterministic steps inserted to facilitate decisions.

In summary, while the empirical and deterministic approaches are very different in scope and application, they can be effectively combined in order to address issues of natural and enhanced attenuation, and issues of time of remediation. 


\subsection{MODELING - REASSEMBLING THE SYSTEM}

How important are the different attenuation mechanisms and processes? In the OSWER Directive on MNA, EPA (1999) recognizes "a variety of physical, chemical, or biological processes that, under favorable conditions, act without human intervention to reduce mass, toxicity, mobility, volume, or concentration of contaminants in soil or groundwater." Further, the directive states that "EPA prefers those processes that degrade or destroy contaminants." As shown below, a parametric evaluation of the various processes supports these general principles and provides results that help develop a more intuitive understanding of the importance of the different attenuation mechanisms. The parametric evaluation is based on a sequence of analytical models in which processes and complexity are added one at a time to determine the relative impact on plume stability and maximum plume size.

A variety of analytical models and scoping calculations have been developed to support waste disposal and contaminant remediation objectives. In some cases these have been used to identify potential sites for storage and isolation of radioactive and/or non-radioactive wastes (e.g., Bailey and Marine, 1980; Rogers and Hung, 1987, Burkholder and Rosinger, 1980, Domenico and Palciauskas, 1982) while others have been used to assess contaminant migration and attenuation (e.g., Wilson and Miller, 1978; van Genuchten and Alvez, 1982; Domenico, 1987; Wexler, 1992).

The simplest models are simple algebraic manipulations of the flow and degradation relationships. An array of variations with increasing complexity based on boundary conditions and processes have also been generated to support individual site needs and conditions. Based on historical data collected on the models used to support

Case Study 6-1. Using Field and Lab Data to Estimate Model Input. In this case study, Clement et al. (2002) use field and lab data to estimate input parameters for BIOCHLOR. MNA for chlorinated solvents, analytical and screening approaches continue to be useful. In a recent survey that focused on real-world MNA experiences since the EPA protocols were developed, $60 \%$ of the respondents indicated that computer models were used to support MNA selection (to show plume stability or to predict remediation time frame or sustainability) and the most frequently used model was the analytical model BIOCHLOR (McGuire et al., 2004).

It is important to note that analytical and semi-analytical models have significant limitations. In order to be valid, each closed form analytical solution requires the subject site to match strict boundary conditions and the assumed waste release scenario. As a result, it is somewhat challenging to apply an analytical solution to a wide range of site-specific conditions. In theory, this would require a large number of separate analytical solutions (for example, see Burkholder and Rosinger, 1980). In practice, the problem has often been simplified by assuming all of the composite degradation processes can be simplified and viewed as first order degradation and that the contaminant source release functions can be assumed to be constant or simple decay functions. 


\section{Case Study 6-1. Using Field and Lab Data to Estimate Model Input}

Using modeling tools to project the behavior and attenuation of chlorinated solvent plumes requires reasonable estimates of all of the various rate parameters. These can be developed based on laboratory study, field measurements, or both. For example, Newell et al. (2002) provide detailed guidance on estimating degradation rate parameters from field data. A particularly clear case study was documented by Clement et al. (2002) in which both laboratory and field data are used to estimate input parameters for the model BIOCHLOR (EPA, 2000; Aziz et al., 2002). Their overall approach is summarized in a flowchart.

The documented approach is notable in several ways. First, an important initial step in the process is a screening step in which the goal is simply determining if biodegradation is occurring. As shown in the case study, this screening step can be based on field observation and supported
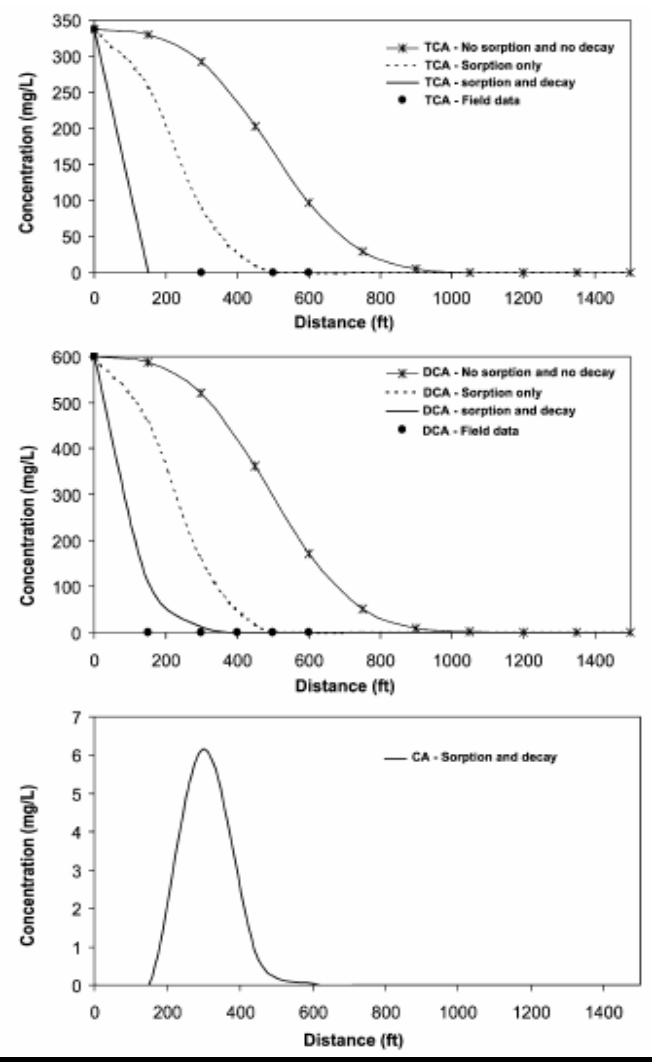

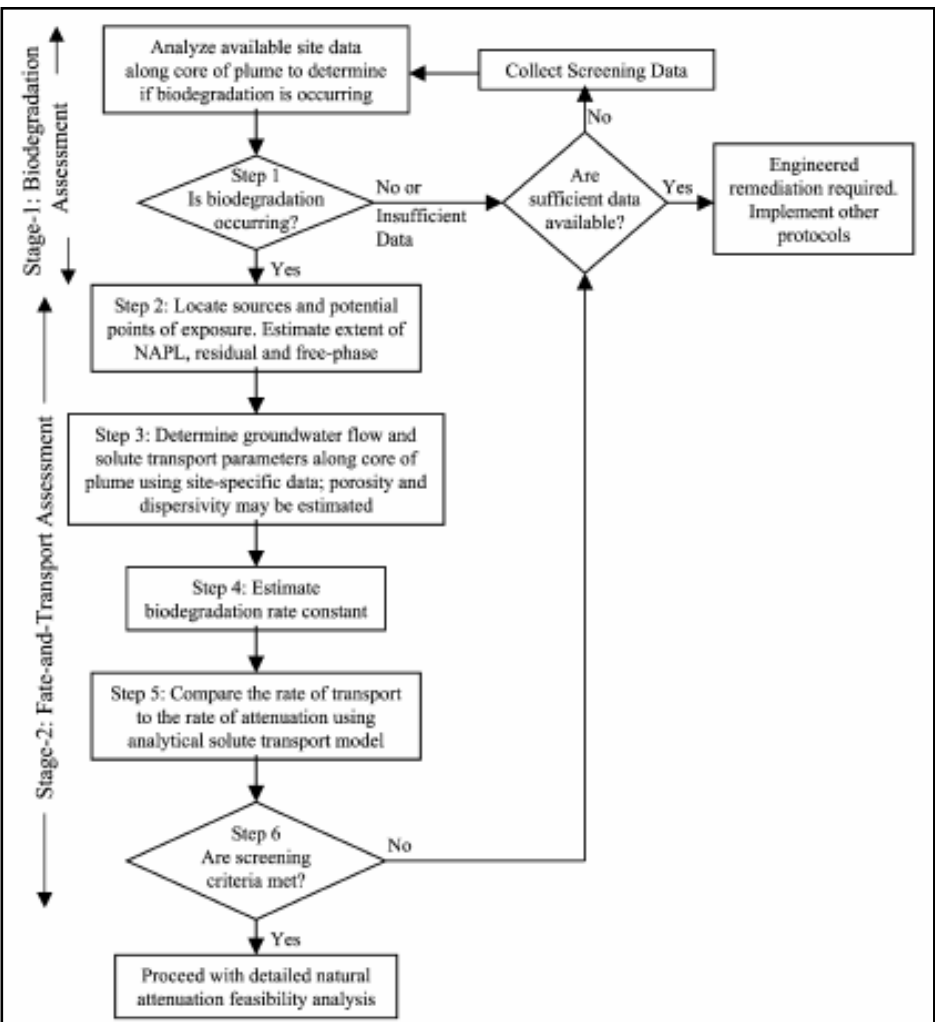

by simple geochemical scoring (e.g., EPA, 1998). The authors then collected and organized the various hydrologic and other physical data needed to develop a site specific model for the site. Preliminary ranges of estimates for the various degradation rate constants, conversion yields, etc., were developed from laboratory and literature sources. Finally, plume projections were completed and calibrated values (from field data) were satisfactorily compared to the initial estimates. The process as shown inspires confidence in the results.

Another nice feature of this case study is the parametric analysis that is inherent in the presentation of the results. In most of the plume projection graphs, the behavior is shown as a function of various combinations of attenuation mechanisms. The results show, for this particular site, the relative influences of dispersion, degradation, and sorption on the transient plume behavior. Parametric evaluation is a powerful tool to improve the conceptual understanding of interrelated processes and this site specific example supports the potential utility of such efforts. 
With respect to MNA/EA, this had led to models such as BIOCHLOR (EPA, 2000; Aziz et al., 2002) and attempts to customize the models based on contaminant specific issues such as competing electron acceptors and amount of available electron donors in the system. These simple models have even been extended to sites where geochemical conditions vary spatially by defining zones of different reaction type or rate. While providing benefit, the extended models are still somewhat limited because they only consider steady-state plumes and only consider 2 zones. Such models cannot be used to model temporary changes in the geochemistry, or transient plume behavior. Notable recent work in improving the rigor and utility of analytical solutions includes the recent work of Falta et al. (2005a and 2005b).

Numerical models that incorporate key MNA/EA processes have also been developed over the years. In some cases, these provide a more flexible alternative to address unusual boundary conditions or time varying conditions. Two of the most widely used numerical models for MNA/EA evaluation are RT3D (Clement, 1997, Clement et al., 1998) and SEAM3D (Secrist, 2002). Like all numerical models, these models require describing the site configuration and conditions mathematically (e.g., by breaking the site into small volumes and solving the mass balance equation for each volume) and estimating attenuation by stepping through time.

Because of the relative simplicity, analytical and screening models are particularly useful for documenting with great clarity the relationships between the primary transport and attenuation processes in a system. These models have the further advantage that one can examine the equations and deduce trends and behaviors for various bounding cases (e.g., very long times). In the following sections, we use such models to examine the relative contributions of various key attenuation mechanisms to plume stabilization. The key mechanisms to be examined are sorption, dispersion, and $1^{\text {st }}$ order decay. The evaluation begins will the simplest relevant case. Then, mechanisms are added sequentially to allow comparison of the resulting estimated plume sizes and structure.

To support the parametric evaluation, an approximate range of the various key model coefficients was tabulated (Table 6-1) based on values reported in the literature (see EPA, 2000; Aziz et al., 2002). For each coefficient, a low, moderate, and high value is provided that generally spans a range of values typically observed. A few additional bounding values are documented in later tables to support the extended analysis. In general, the parametric analysis provides a generic description of the behavior of a plume as a function of the various parameters. The process also generates a specific range of plume sizes for the bounding combinations of parameters from Table 6-1. As a result, this process will help assess the relative significance of, and interactions among, the various attenuation mechanisms and provide insight into the actual physical size of plumes controlled by attenuation. 


\section{Case Study 6-2. A Zonation Approach to Heterogeneity}

A significant and relatively common challenge when quantifying contaminant mass balance is the effect of heterogeneity. This heterogeneity takes many forms - physical heterogeneity such as vertical layering and lateral facies changes, and chemical heterogeneity such as geochemical gradients and varying environments along the flow path (e.g., aerobic groundwater entering wetlands in the hyporheic zone). A straightforward and logical way to address this problem is to develop a site specific conceptual model of the heterogeneity and then define different zones that are characterized by different physical and/or biogeochemical parameters. Typically this has been done using a numerical model - the discretized gridblocks or cells in the various model zones are assigned the relevant parameters. For example, if a site has an anaerobic core surrounded by aerobic groundwater, then the appropriate rates for reductive or oxidative conditions can be assigned as appropriate. This particular case is relatively common and might demonstrate synergy because the parent chlorinated solvents typically degrade fastest under reducing conditions while many daughters degrade rapidly under the surrounding aerobic conditions. As shown below, Clement et al. (2000) used RT3D to evaluate just such a case for a site at Dover Air Force Base.
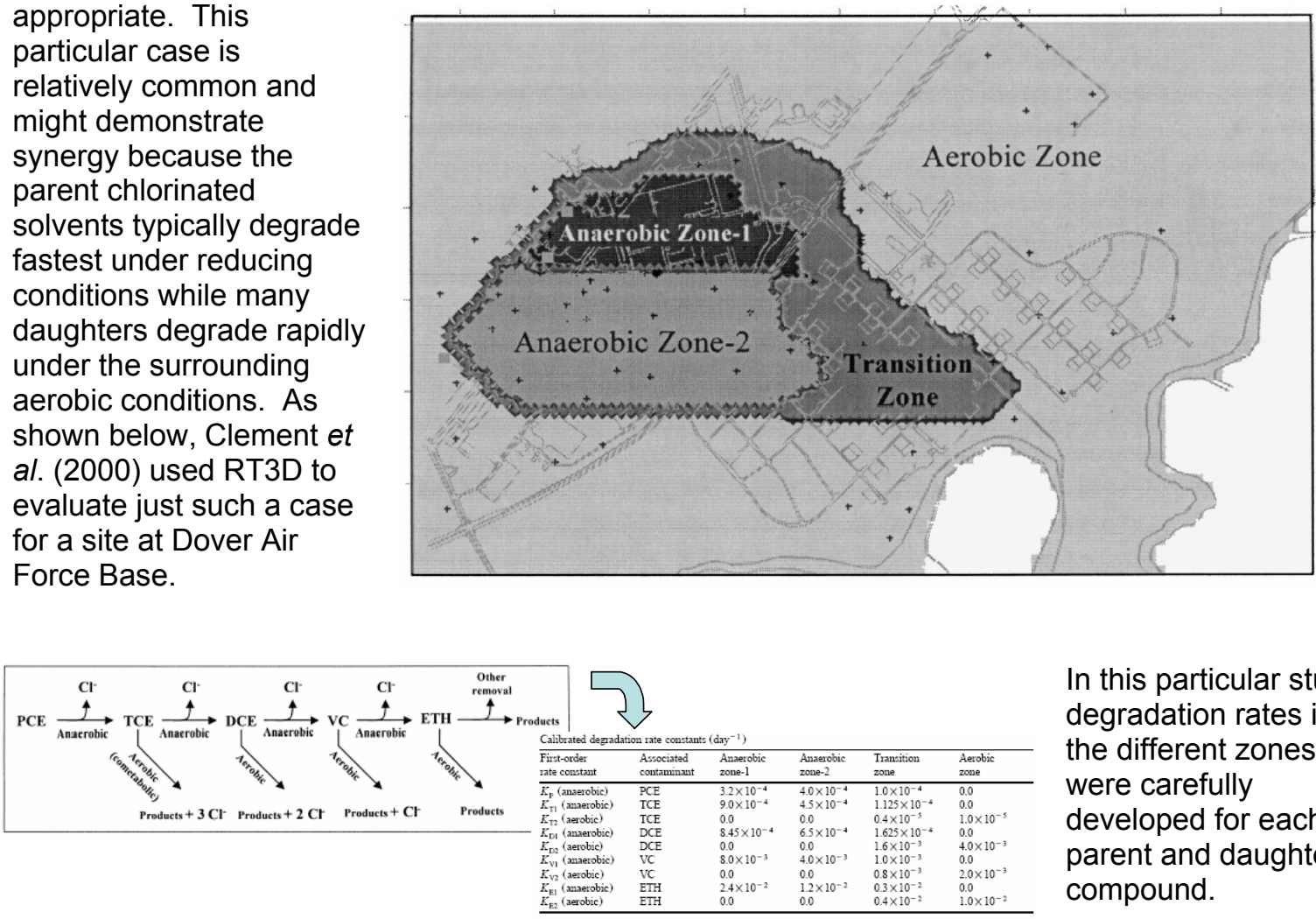

In this particular study, degradation rates in the different zones were carefully developed for each parent and daughter compound.

The numerical modeling framework allowed these rates to be combined with the site hydrology to provide plume projections, mass balance estimates, and to support a sensitivity/uncertainty analysis. The model showed the importance of accounting for the differences in geochemistry in the different zones - in particular, the mass balance and projected plumes were very sensitive to degradation of the parent solvents in the anaerobic zones and the continued degradation of daughters in the surrounding zones. This type of zonation based modeling to deal with macroscale heterogeneity is a powerful tool and should be encouraged. Notably, in response to this need, the latest version of BIOCHLOR (Aziz et al., 2002) incorporates an option of zonation in the plume flow path. This option does not appear to be widely known or used, however, because no literature or conference proceedings examples could be identified. The challenges of heterogeneity, both at the macroscale and microscale, remain one of the most important targets for thought and creativity when modeling and understanding MNA/EA. 
Table 6-1. Key Site Parameters that Influence MNA/EA

\begin{tabular}{|l|c|c|c|}
\hline & $\begin{array}{c}\text { groundwater seepage } \\
\text { velocity, } \\
\mathrm{v}_{\mathrm{s}}\end{array}$ & $\begin{array}{c}\text { contaminant retardation } \\
\text { factor, } \\
\mathrm{R}=\mathrm{v}_{\mathrm{s}} / \mathrm{v}_{\mathrm{c}} \\
=1+\left(\mathrm{K}_{\mathrm{d}} \rho_{\mathrm{b}} / \mathrm{n}\right) \\
(\mathrm{m} / \mathrm{yr})\end{array}$ & $\begin{array}{c}1^{\text {st }} \text { order contaminant } \\
\text { degradation rate } \\
\text { constant in plume, } \\
\lambda\end{array}$ \\
\hline Low & 10 & 1 & $1 / \mathrm{yr})$ \\
\hline Moderate & 25 & 1.5 & $10^{-1}$ \\
\hline High & 100 & 5 & 5 \\
\hline
\end{tabular}

For clarity and brevity, the following modeling sections have been presented in summary fashion. Appendix A provides greater detail of the mathematical derivations summarized in the following sections.

\subsection{INTERACTION OF SORPTION AND DEGRADATION}

A case documenting the interrelationship between flow rate, sorption, and degradation demonstrates the general value of a parametric approach in developing a deeper understanding of the interrelationships between transport and attenuation processes. Such a case also provides insight into the relative significance of the included processes and parameters. A simple algebraic analysis that accounts for groundwater flow rate, linear sorption, and first order decay provides insight into the relationships between these parameters. This analysis assumes that the primary driving force for moving a dissolved contaminant from one location to another is flow of groundwater and neglects the complicating effects of dispersion. Under these boundary conditions and assumptions the plume migration is "plug flow" and there is a simple relationship between the travel distance and travel time:

$$
x=\frac{v_{s}}{R} t=v_{c} t
$$

Since the contaminant is assumed to degrade according to first order kinetics $(\lambda>0)$, the relationship between concentration and time is:

$$
\left(\mathrm{C} / \mathrm{C}_{0}\right)=\mathrm{e}^{-\lambda \mathrm{t}}
$$


If the concentration at the source is assumed to be constant and inexhaustible, then the steadystate concentration at any travel distance can be determined by algebraically combining these equations:

$$
x=\frac{v_{s}}{R \lambda} \ln \left(\frac{C}{C_{0}}\right)=-L_{\lambda} \ln \left(\frac{C}{C_{0}}\right)
$$

This simple equation allows the creation of a graph that relates the concentration, $\left(\mathrm{C} / \mathrm{C}_{0}\right)$, to travel distance downgradient of the source (Figure 6-1). The equation and graph indicate that, under the influence of $1^{\text {st }}$ order degradation, flow, and linear sorption, the steady-state concentration decreases as a function of increasing travel distance away from a constantinexhaustible source. A higher groundwater flow rate results in a larger steady-state plume, while greater sorption/retardation and faster degradation result in a smaller steady-state plume. Moreover, plume behavior can be completely predicted using the reduced variable, $\mathrm{v}_{\mathrm{s}} /(\mathrm{R} \lambda)$ which has units of length (e.g., meters). The reduced variable, designated $\mathrm{L}_{\lambda}$, is a characteristic length that controls the steady-state plume size and structure. The calculated travel distance to the point where the concentration is approximately half of the original is $0.693 \mathrm{~L}_{\lambda}$. According to this calculation, any combination of $\mathrm{v}_{\mathrm{s}}, \mathrm{R}$ and $\lambda$ that yield a particular value of $\mathrm{L}_{\lambda}$ will result in identical steady-state plume size.

Figure 6-1a shows the general relationship between relative concentration and travel distance for a plume emanating from a constant source for a broad range of conditions. Figure 6-1b provides a practical example of how to interpret the parametric graph. If the required concentration reduction is a factor of 1,000 (i.e., $\mathrm{C} / \mathrm{C}_{0}=0.001$ ) and $\mathrm{L}_{\lambda}$ is 3 , then the steadystate plume will extend approximately $20 \mathrm{~m}$ downgradient from the source. Closer to the source the concentration will be higher than the target and further from the source the concentration will be lower than the target. It is clear from this figure that the following additional parameters (Table 6-2) will be useful in performing the parametric evaluation. After all of the various attenuation factors are evaluated in the following sections, an overall summary table of plume size for the three bounding cases will be provided for comparison.

Table 6-2. Bounding values for remediation goals and the derived parameter $\left(L_{\lambda}\right)$

\begin{tabular}{|l|c|c|}
\hline & $\begin{array}{c}\text { Required concentration } \\
\text { reduction factor to meet goal } \\
\mathrm{C} / \mathrm{C}_{0}\end{array}$ & $\begin{array}{c}\text { characteristic transport length } \\
\text { for bounding calculations, } \\
\mathrm{L}_{\lambda}=\left(\mathrm{v}_{\mathrm{s}} / \mathrm{R} \lambda\right) \text { or }=\left(\mathrm{v}_{\mathrm{s}} / \lambda\right), \\
\text { see paragraph below } \\
(\mathrm{m})\end{array}$ \\
\hline best case & $(--)$ & 0.4 \\
\hline intermediate case & $10^{-1}$ & 17 \\
\hline worst case & $10^{-2}$ & 1000 \\
\hline
\end{tabular}




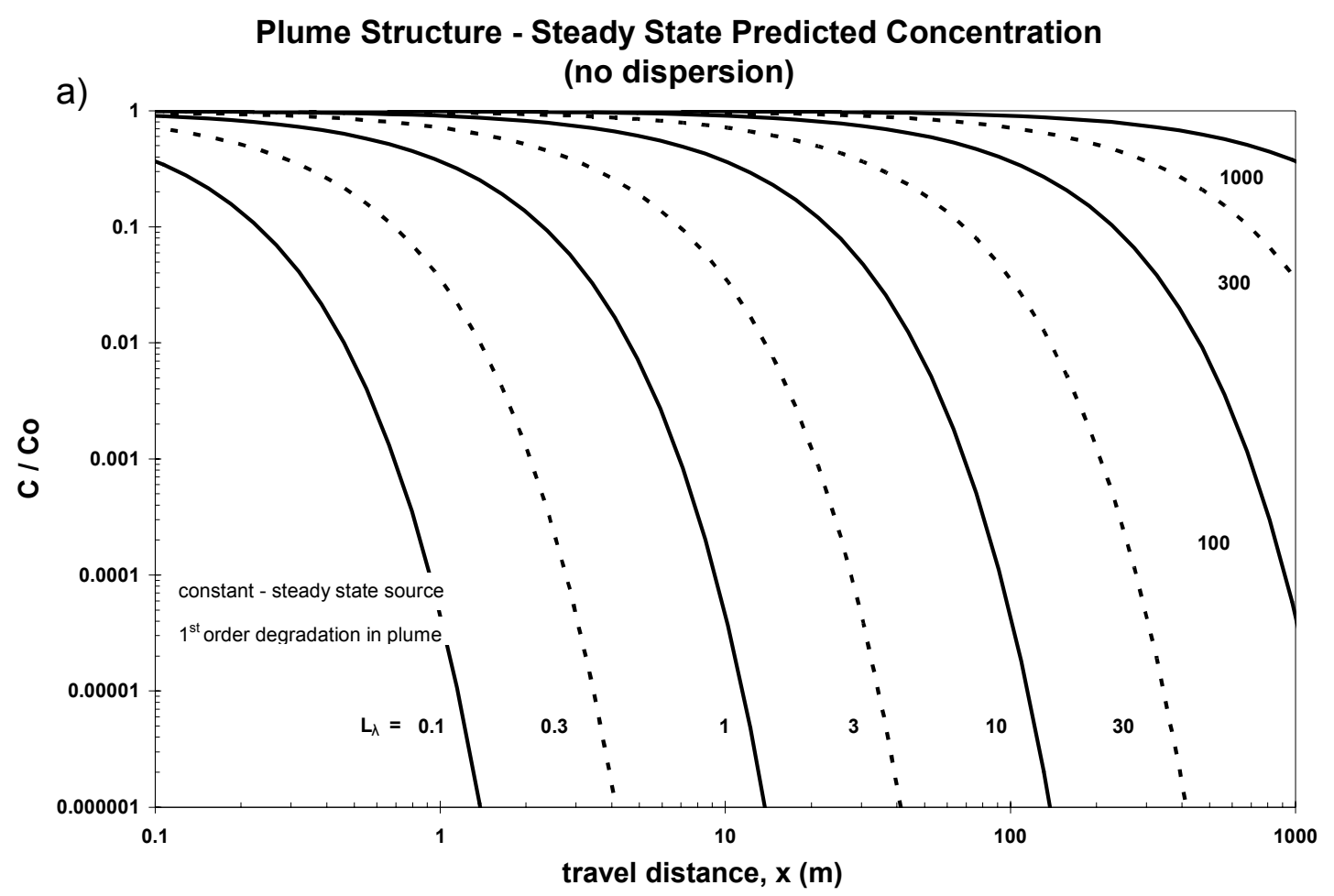

b) Example Stable Plume Size for $L_{\lambda}=3$ and a desired $C / C_{0}$ of 0.001

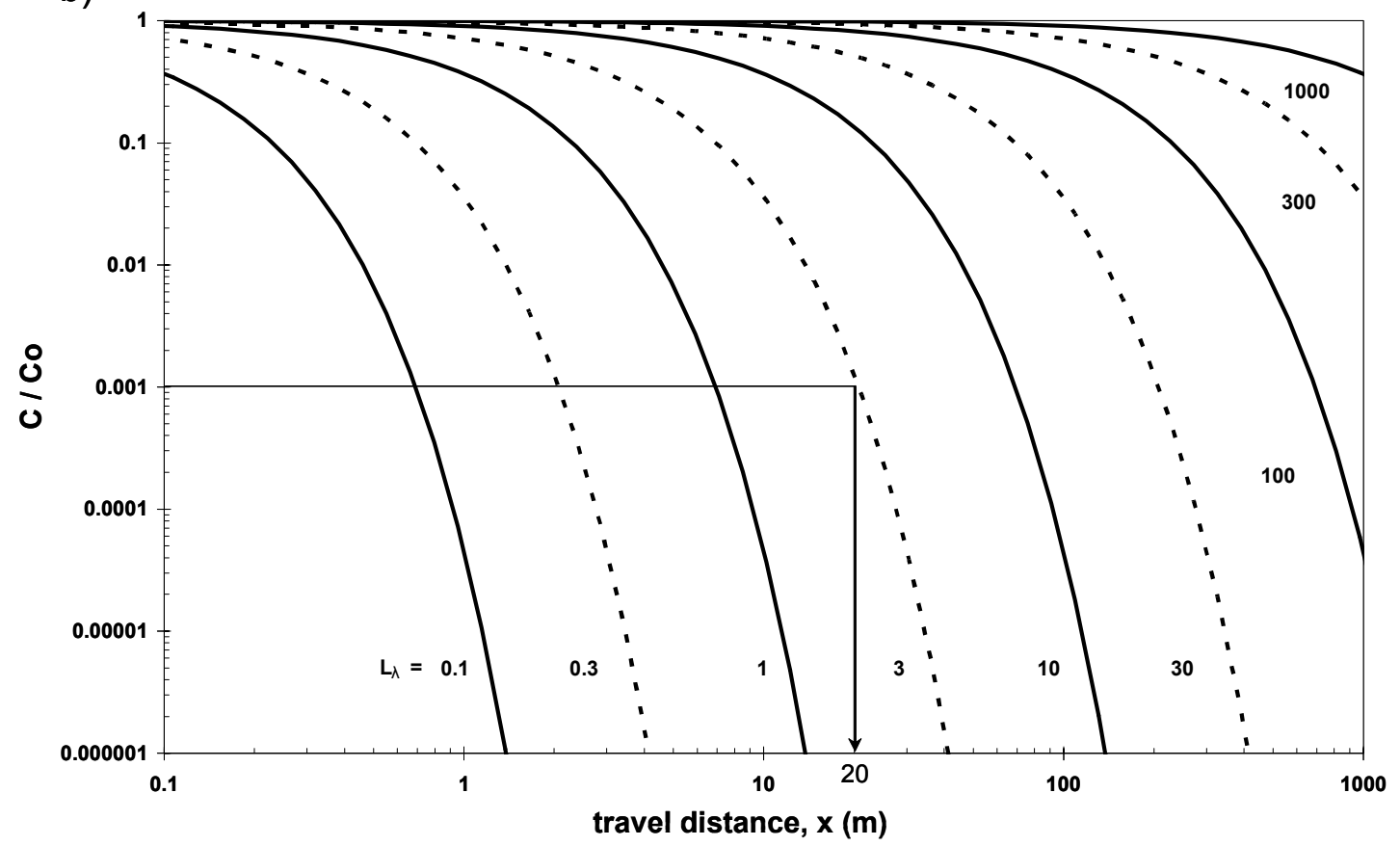

Figure 6-1. Plume size and structure for a 1D plume influenced only by degradation and sorption. a) generic results, b) example case 
The BIOCHLOR (EPA 2000; Aziz et al. 2002) model and associated recommendations for estimating degradation parameters from field data (Newell et al., 2002) assume that solvent degradation only occurs in the aqueous phase. This assumption is reasonable and widely held but has not been confirmed with definitive supporting scientific literature. This issue is addressed in more detail in Case Study 6-3. Importantly, with a few exceptions that are noted, this alternative assumption can be accommodated by the parametric analysis by appropriate definition of $\mathrm{L}_{\lambda}$. The various parametric graphs are then interpreted using whichever definition is consistent with the desired assumption.

If first order degradation occurs in both phases: $\mathrm{L}_{\lambda}=\mathrm{v}_{\mathrm{s}} /(\mathrm{R} \lambda)$ If first order degradation occurs only in the aqueous phase: $\mathrm{L}_{\lambda}=\mathrm{v}_{\mathrm{s}} / \lambda_{\mathrm{a}}$

\subsection{EFFECTS OF DISPERSION AND SOURCE DECAY - METHOD DEVELOPMENT}

The conservative calculation provided above, accounting for only first order decay and sorption (as appropriate), is similar to a portion of the evaluation performed by Bailey and Marine (1980) in their detailed sensitivity study of the effects of varying flow rate, dispersion coefficients, and other parameters on the concentration profiles of contamination in plumes emanating from waste disposal sites. The algebraic calculation above does not account for the depletion of the source over time nor the dilution effects caused by the dispersion of the contaminant away from the plume centerline. These factors are left constant in a worst-case configuration to allow reasonable assessment of the importance of the included primary factors. This simple calculation does not follow the formation and fate of daughter products and does not take credit for the lowering of concentrations that result from the size, shape, or mass depletion within the source zone. More complex analytical solutions, hybrid approaches and numerical models that allow projections that account for the desired additional factors are available and can be used to refine the estimates in a step-by-step manner.

In order to add these additional factors, a brief review of the structure and key details of the relevant analytical models is needed. In this application, the analytical model is simply a tool to simulate the concentration of a contaminant in a contaminant plume. The input information includes the initial conditions and boundary conditions, information on the contaminant source, and information on the processes that degrade or dilute the contamination. The options that are available to represent the introduction of a contaminant into a system (i.e., the source) provide a good example of the type of information that is used to generate the analytical solution. The source can be assumed to be: a constant concentration $\left(\mathrm{C}_{(0,0,0, t)}=\mathrm{C}_{0}\right)$; an exponentially decaying source $\left(\mathrm{C}_{(0,0,0, t)}=\mathrm{C}_{0} \mathrm{e}^{-\mathrm{k}_{\mathrm{s}} \mathrm{t}}\right)$ ); a constant flux, or any of a number of other variations that include details of source length, and/or decay toward nonzero values. Most analytical models that are in use assume either a constant and inexhaustible source (worst case) or an exponentially decaying source (as a more realistic case). The contaminant is then assumed to enter a plume that is subject to a variety of influences as shown in Table 6-3.

A more complete discussion of model structure and development, including alternative boundary conditions and alternative solutions, is provided in Chapter 8 of Wiedemeier et al. (1999). 
Table 6-3. Summary of Some of the Available Analytical Models

\begin{tabular}{|l|c|l|l|}
\hline \multicolumn{1}{|c|}{ References } & Dimensions & \multicolumn{1}{c|}{ Source } & \multicolumn{1}{c|}{ Processes } \\
\hline $\begin{array}{l}\text { Bear (1972), } \\
\text { Burkholder and } \\
\text { Rosinger (1980), } \\
\text { vanGenuchten and }\end{array}$ & 1D & $\begin{array}{l}\text { Constant and } \\
\text { inexhaustible }\end{array}$ & $\begin{array}{l}\text { Advection, dispersion, linear } \\
\text { sorption, and degradation during } \\
\text { transport (first order) }\end{array}$ \\
\hline $\begin{array}{l}\text { Wurkholder and } \\
\text { Rosinger (1980), } \\
\text { vanGenuchten and } \\
\text { Alves (1982) }\end{array}$ & 1D & $\begin{array}{l}\text { Decaying } \\
\text { Source term }\end{array}$ & $\begin{array}{l}\text { Advection, dispersion, linear } \\
\text { sorption, and degradation during } \\
\text { transport (first order) }\end{array}$ \\
\hline $\begin{array}{l}\text { Wilson and Miller } \\
\text { (1978) }\end{array}$ & 2D & $\begin{array}{l}\text { Constant and } \\
\text { inexhaustible }\end{array}$ & $\begin{array}{l}\text { Advection, dispersion, linear } \\
\text { sorption, and degradation during } \\
\text { transport (first order) }\end{array}$ \\
\hline $\begin{array}{l}\text { Domenico (1987) } \\
\text { Falta } \text { et al. (2005a } \\
\text { and 2005b) }\end{array}$ & 3D & $\begin{array}{l}\text { Constant and } \\
\text { inexhaustible }\end{array}$ & $\begin{array}{l}\text { Advection, dispersion, linear } \\
\text { sorption, and degradation during } \\
\text { transport (first order) }\end{array}$ \\
\hline $\begin{array}{l}\text { Aziz } \text { et al. (2002), } \\
\text { modified from } \\
\text { Domenico (1987) }\end{array}$ & 3D & $\begin{array}{l}\text { Decaying } \\
\text { Source term }\end{array}$ & $\begin{array}{l}\text { Advection, dispersion, linear } \\
\text { sorption, and degradation during } \\
\text { transport (first order) }\end{array}$ \\
\hline 3D & $\begin{array}{l}\text { Decaying } \\
\text { and/or } \\
\text { partially } \\
\text { remediated } \\
\text { source at a } \\
\text { defined time }\end{array}$ & $\begin{array}{l}\text { Advection, dispersion, linear } \\
\text { sorption, and degradation during } \\
\text { transport (first order) }\end{array}$ \\
\hline
\end{tabular}

An interesting feature of analytical models is that the influence of the various assumed processes can be deduced by examining the structure of the mathematical equations. In most cases, a more complex solution can be shown to be identical to a simpler solution by selecting the appropriate parameter value (for example a 2D solution will equal a 1D solution if the transverse dispersion coefficient is set to 0 ). As a result, a useful equation for our parametric evaluation is the simple but relatively complete modified solution of Domenico (1987) and Aziz et al. (2002). With this solution, we will selectively set the parameters to sequentially examine the relative contribution of various attenuation mechanisms in terms of plume size and structure. Figure 6-2 shows the geometry of the solution and boundary condition and defines a few key variables.

Note that recent papers suggest that the Domenico (1987) solution may be subject to inaccuracies under some conditions (due to using a specific concentration boundary condition rather than a specified flux boundary condition). Alternative formulations with improved mass conservation have been developed by several researchers including Falta et al. (2005a and 


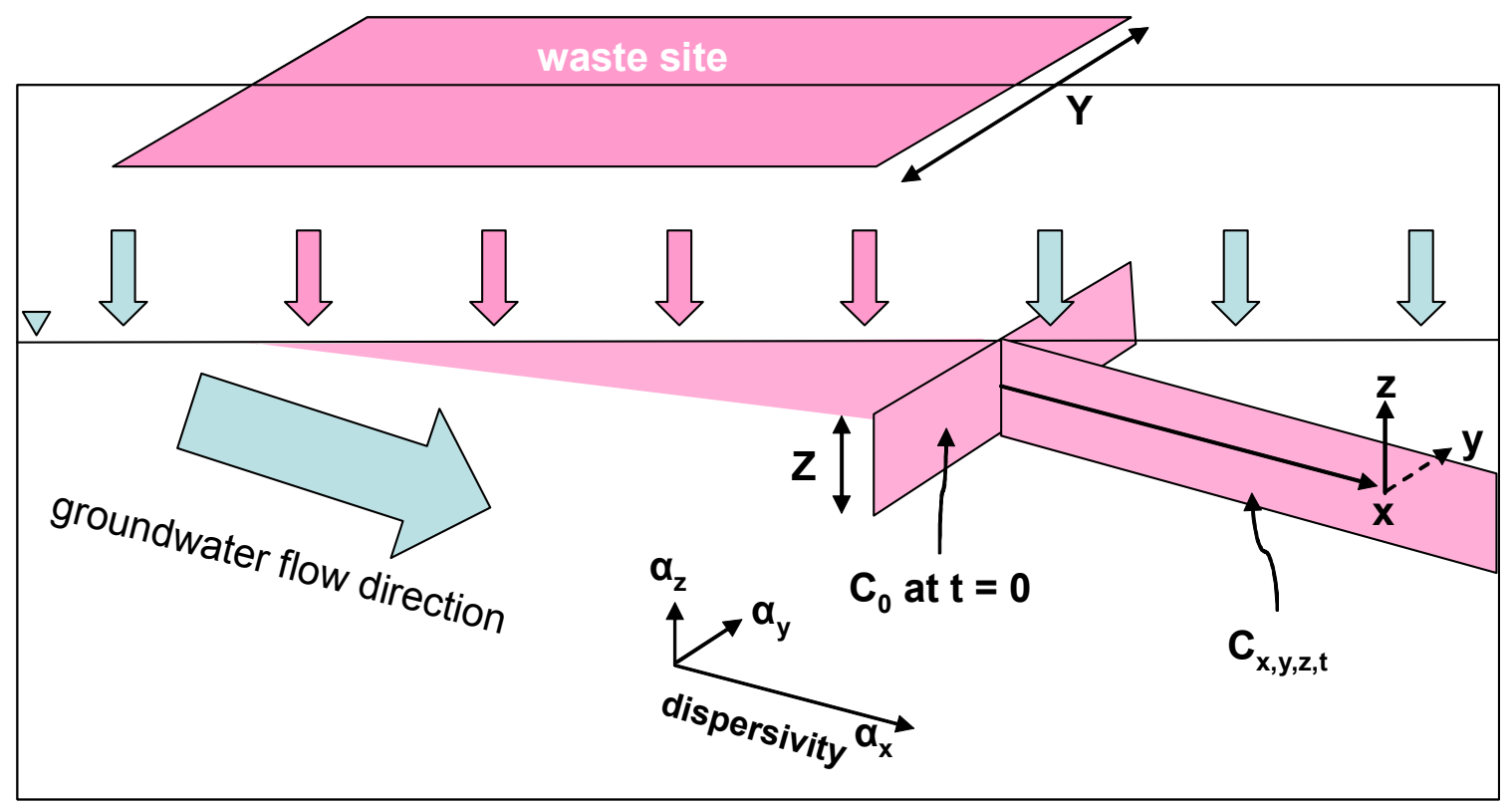

Figure 6-2. General Geometry and Nomenclature for Analytical Solution

2005b) and Clement et al. (2006). The inaccuracies are generally small under most conditions and we have selected the Domenico (1987) equations for our parametric study because of its simplicity and to be as consistent as possible with historical MNA modeling.

The resulting mathematical solution for this situation is well behaved and relatively straightforward:

$$
C_{(x, y, z, t)}=C_{0} e^{-k_{s} t} \frac{f_{x}}{2} \frac{f_{y}}{2} \frac{f_{z}}{2}
$$

See Appendix B for a more detailed discussion of Equation 3.

The first term of this equation is the initial source concentration with an exponential to describe a decreasing source over time. If the source is constant and inexhaustible, then $\mathrm{k}_{\mathrm{s}}$ is set to 0 and the equation becomes identical to Domenico (1987). Each of the following terms describes the attenuation due to dispersion and degradation. The primary term, $\mathrm{f}_{\mathrm{x}}$, accounts for advection, longitudinal dispersion and first order decay. The remaining terms account for dispersion only in the lateral ( $y$ and $z$ ) directions, respectively. Each of the terms $f_{x}, f_{y}$ and $f_{z}$ range from 0 (if the term accounts for a lot of attenuation) to 2 (if the term does not account for any substantive attenuation). Each term is divided by 2 to determine the contributory impact of the process(es) in attenuating the contaminant concentration. For example, if we want to assume minimal lateral dispersivity we would assume the coefficient of lateral transverse dispersivity, $\alpha_{y}$, is small. At small values of $\alpha_{y}$ the value of $f_{y} \approx 2$. Thus $f_{y} / 2 \approx 1$ and lateral dispersivity does not modify the calculated concentration, i.e., it does not contribute to attenuation. 
Note that the version of the equations we selected (Appendix B differ slightly from Aziz et al. (2002). The version presented herein allows degradation to occur in both the aqueous and sorbed phases and assumes the degradation rate constant, $\lambda$, has been defined and measured consistent with that assumption. This was done to simplify the equation and help clarify relationships. In practice, values of $\lambda$ ( or $\lambda_{\mathrm{a}}$ ) are estimated from field or lab data and the modeling results will be insensitive to what is assumed as long as the same assumption is made when calculating $\lambda$ (or $\lambda_{a}$ ) and then using the term in models. In fact, the two models generate identical results when $\lambda=\lambda_{\mathrm{a}} / \mathrm{R}$, a condition that would automatically result from estimating the coefficient from field based plume data. Technically, this uncertainty primarily impacts the ability to relate laboratory data to the field, to link site mineralogy to the degradation process, and to apply past radionuclide models to contaminants that are assumed to degrade by first-order kinetics.

\subsection{EFFECTS OF LONGITUDINAL DISPERSION}

Using equation 3 and setting $\mathrm{k}_{\mathrm{s}}=0$ (constant source) and $\mathrm{f}_{\mathrm{y}} / 2=\mathrm{f}_{\mathrm{z}} / 2=1$ we can examine the incremental effects of longitudinal dispersion on the plug flow case presented earlier. The resulting equation corresponds to the equations for a 1D steady-state plume developed earlier by Bear (1972) and others.

\section{See Appendix B for a more detailed presentation of the mathematical formulation used to evaluate longitudinal dispersion.}

Figure 6-3 is the graph that relates the concentration, $\left(C / C_{0}\right)$, to travel distance downgradient of the source. As with the plug flow case, the graph indicates that, under the influence of $1^{\text {st }}$ order degradation, flow, longitudinal dispersion, and linear sorption, the steady-state concentration decreases as a function of increasing travel distance away from a constant-inexhaustible source. Similar to the plug flow case, the plume behavior can be completely predicted using the reduced variable, $\mathrm{L}_{\lambda}$. Figure 6-3a shows the general relationship between relative concentration and travel distance for a plume emanating from a constant source for a broad range of conditions and includes the previous example for comparison. If the required concentration reduction is a factor of 1,000 (i.e., $\mathrm{C} / \mathrm{C}_{0}=0.001$ ) and $\mathrm{L}_{\lambda}$ is 3 , then the steadystate plume will extend approximately $34 \mathrm{~m}$ downgradient from the source. Note that this is actually larger than the "conservative" plug flow case!

The results indicate that for a steady-state case with a constant source concentration, dispersion increases the plume size relative to a baseline plug flow case. Upon examination, this result makes sense; dispersion spreads the contamination ahead of bulk flow before it has had time to decay. Figure 6-3b shows the effect in a more general fashion. The steady-state longitudinal 


\section{Case Study 6-3. What if Degradation Is Only in the Aqueous Phase?}

The dominant processes in natural attenuation and risk reduction generally involve chemical or biological destruction or stabilization - subsurface redox processes are a particularly significant class of biological or chemical process. EPA, when formulating BIOCHLOR (EPA 2000, Aziz et al., 2002) assumed the sorbed phase is not subject to degradation. This assumption is distinctly different from models developed for radionuclide transport in which the contaminant half-life is a fundamental physical property that is the same in all contaminant phases and forms. Thus, the large body of literature related to plume transport undergoing first order decay processes that has been developed by scientists studying radionuclides may not be entirely applicable to crossover application for other contaminants. Recent EPA guidance on parameter estimation for chlorinated solvents (Newell et al. 2002) reconfirmed their recommendation that one should initially assume that the sorbed contaminant is not undergoing degradation. A quick survey of the literature for such contaminants suggests that many current models assume the sorbed contaminant is not subject to degradation (e.g., BIOCHLOR) while others assume the opposite or provide the user an option (e.g., RT3D and SEAM3D)). There is only modest scientific data in the literature to support which approach is correct or to define the conditions under which each of the alternative assumptions should be made. This knowledge gap limits the ability to relate laboratory measured kinetics to the field and to properly project critical MNA related plume dynamics such as stability and maximum size.

The steady-state plume structure figure below illustrates the significance of this assumption. Compared to degradation in the aqueous phase only, degradation in both the aqueous and sorbed fractions directly reduces the maximum predicted plume size. For a retardation factor of 3 , a plume with both aqueous and sorbed phase degradation will stabilize at $1 / 3^{\text {rd }}$ the size of a plume that is undergoing only aqueous degradation. Additional research in this area over the next several years will be helpful in developing a technically based assessment of the relative rates of degradation of the sorbed versus the aqueous contaminant under a variety of site specific conditions.

\section{Steady State Plume Structure}

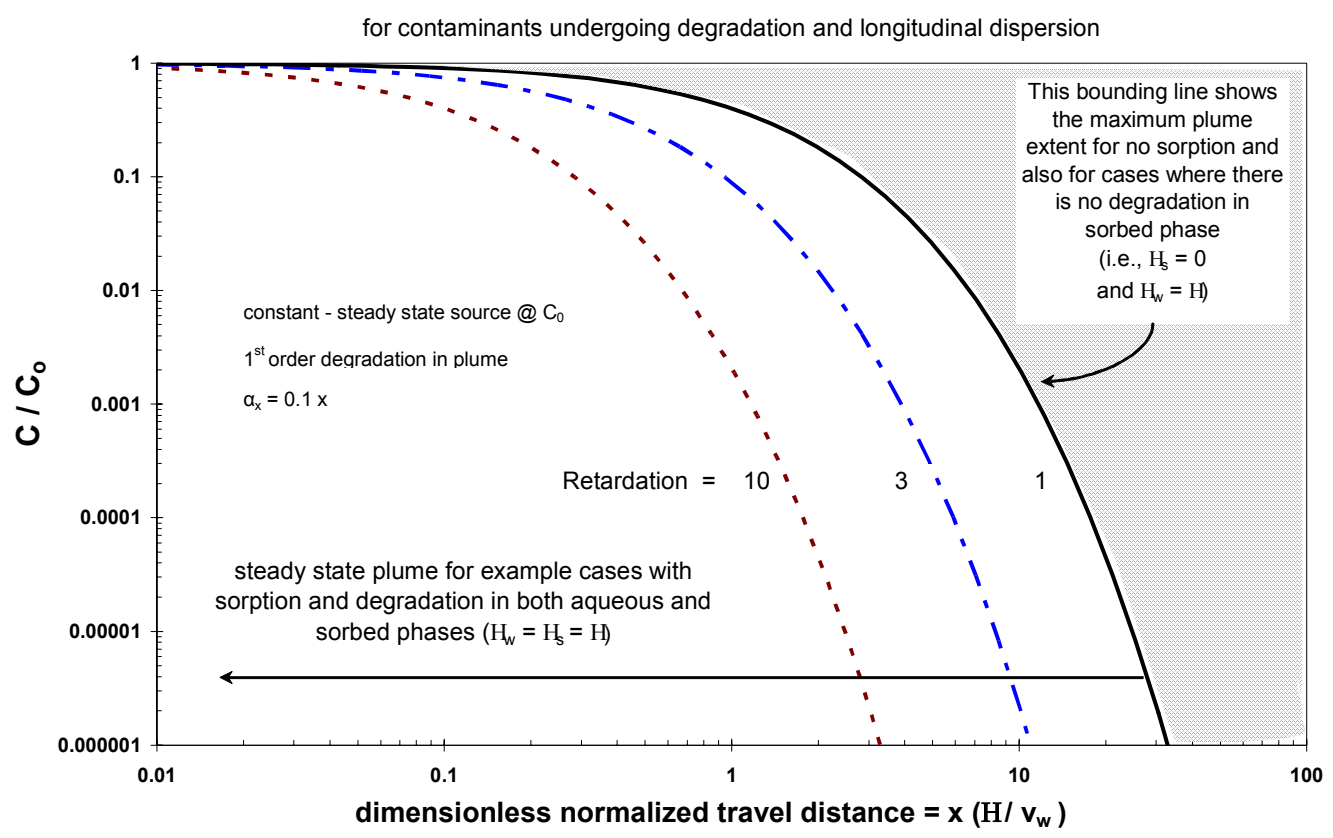



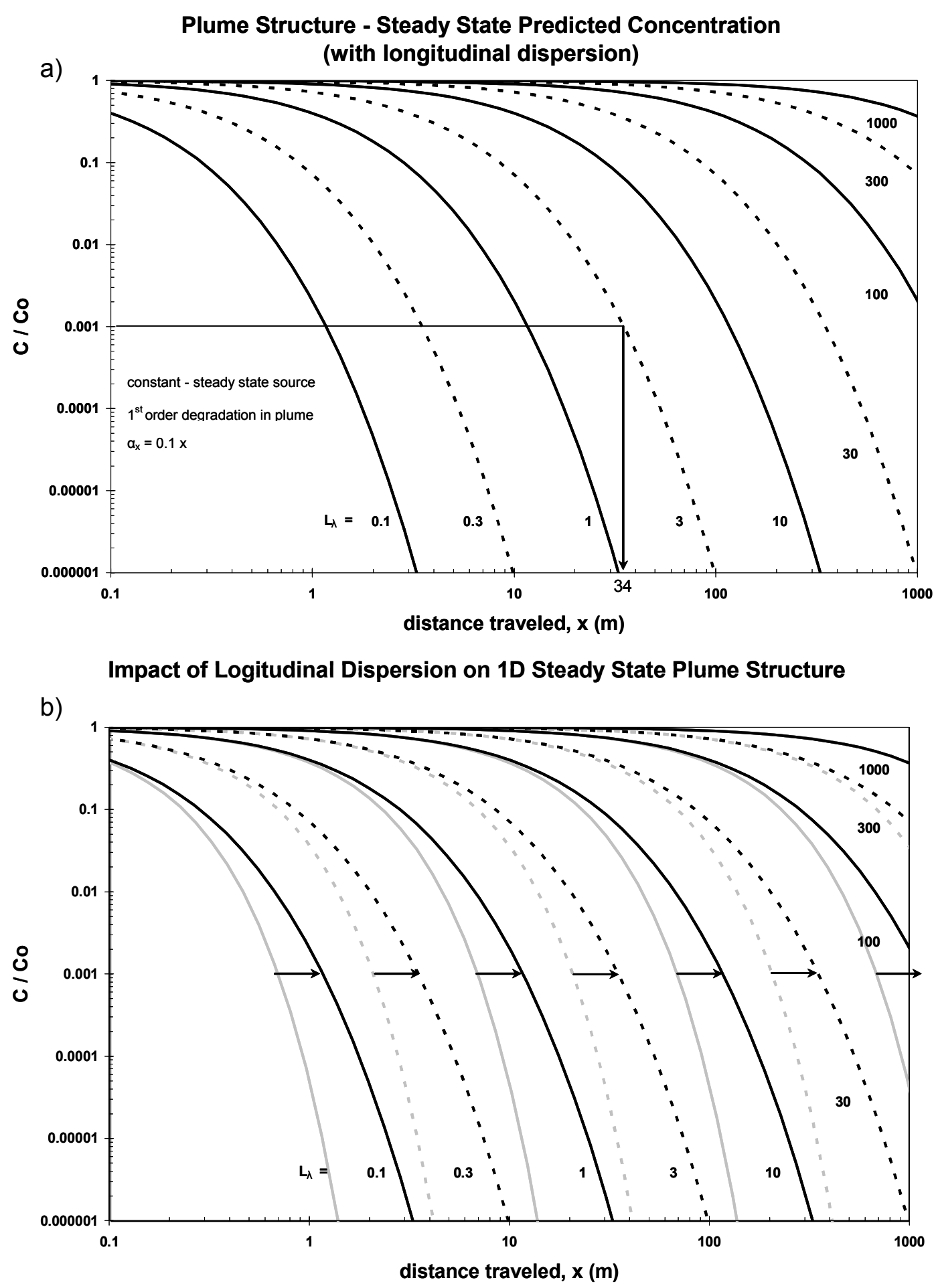

a) generic results and example case

b) incremental impact of longitudinal dispersion (underlay is plug flow case)

Figure 6-3. Plume size and structure for a 1D plume influenced by degradation, sorption, and longitudinal dispersion 
dispersion case is shown as a dark overlay placed on top of the plug flow results (gray underlay). For a constant and inexhaustible source and any particular $\mathrm{L}_{\lambda}$, longitudinal dispersion results in a larger steady-state plume (i.e. higher concentration for all flow distances).

\subsection{EFFECTS OF SOURCE DECAY}

Field data suggest that contaminant sources, even sources of chlorinated solvents, are not constant and inexhaustible (Newell and Adamson, 2005). Using equation 3 and setting $\mathrm{k}_{\mathrm{s}} \neq 0$ (decaying source) and $\mathrm{f}_{\mathrm{y}} / 2=\mathrm{f}_{\mathrm{z}} / 2=1$ we can examine the incremental effects of a decaying source on the longitudinal dispersion case presented above.

\section{See Appendix B for a more detailed presentation of the mathematical formulation used to evaluate source decay.}

Note that while this is still a 1D solution, it is no longer steady-state. The concentrations at any distance are transient. For every $\mathrm{x}$, the concentrations increase and then decrease and the concentrations do not stabilize at a constant value. For our parametric evaluation we used a value near the maximum source decay rate allowed by the Domenico model, i.e., $\lambda=\mathrm{k}_{\mathrm{s}}$. Since the analytical solution is not steady-state, we are now identifying the maximum concentration that is projected for any time at each flow distance. This maximum concentration will occur at a different time at each $\mathrm{x}$, but it is this transient maximum concentration that is the diagnostic indicator of plume growth/size for purposes of natural attenuation modeling.

Similar to the earlier figures, Figure 6-4 for the decaying source model relates the maximum concentration, $\left(\mathrm{C} / \mathrm{C}_{0}\right)$, to travel distance downgradient of the source. The graph indicates that the projected maximum concentration decreases as a function of increasing travel distance. Similar to the earlier cases, the plume behavior can be generally predicted based on the reduced variable, $\mathrm{L}_{\lambda}$. Figure 6-4a shows the general relationship between relative concentration and travel distance for a plume emanating from a constant source for a broad range of conditions and includes the previous example for comparison. If the required concentration reduction is a factor of 1,000 (i.e., $\mathrm{C} / \mathrm{C}_{0}=0.001$ ) and $\mathrm{L}_{\lambda}$ is 3 , then the steadystate plume will extend approximately $25 \mathrm{~m}$ downgradient from the source. Note that this example result is between the two cases documented above.

Figure $6-4 \mathrm{~b}$ shows the incremental effect in a more general fashion. The transient source decay case is shown as a dark overlay placed on top of the steady-state longitudinal dispersion

See Case Study 6-4. In this case study, Falta and others develop modeling tools and apply them to the problem of determining the value of source remediation. results (gray underlay). For any particular $\mathrm{L}_{\lambda}$, source decay results in a smaller plume (i.e., lower maximum concentration for all flow distances). 


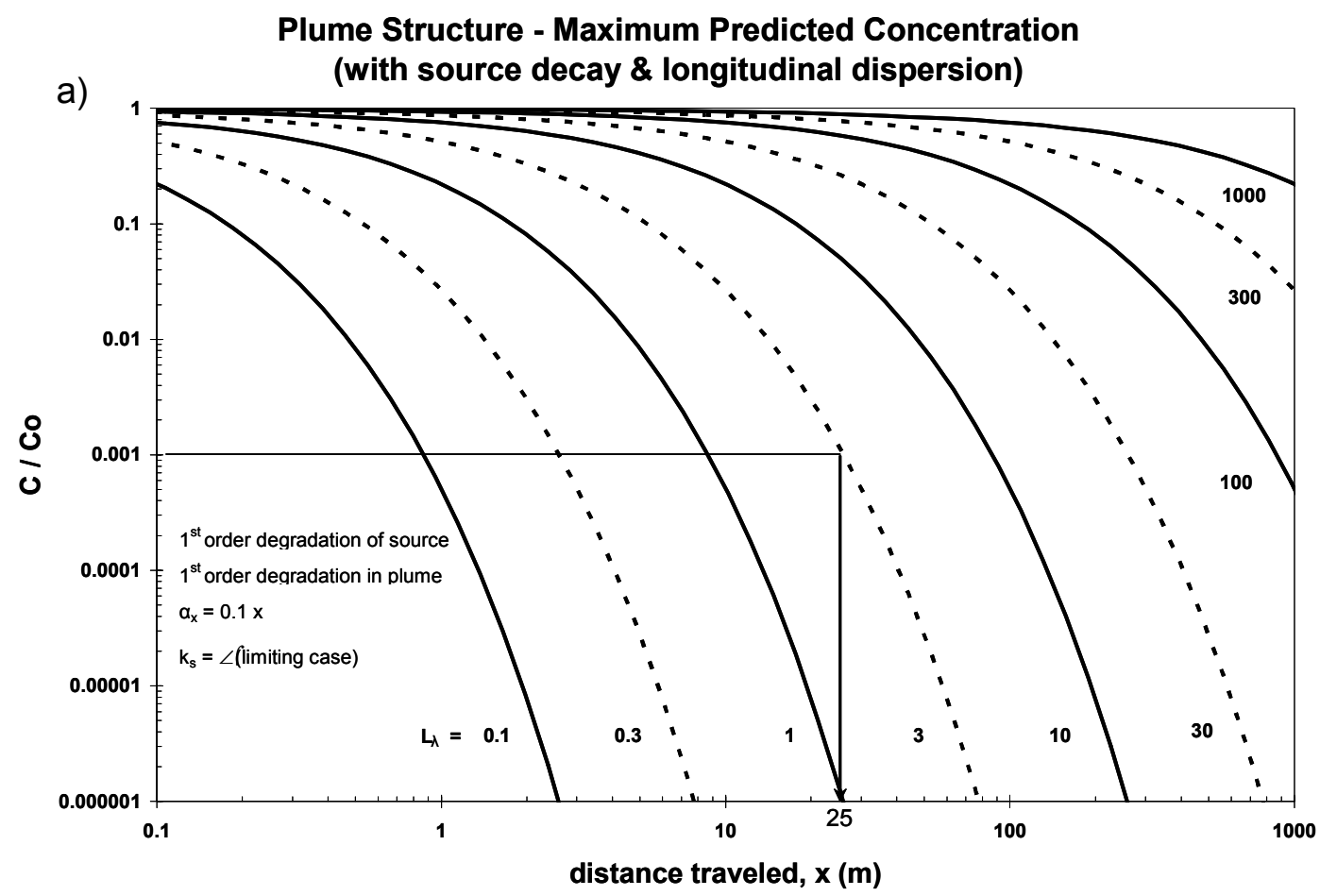

Impact of Source Decay on Maximum Predicted Concentration

b)

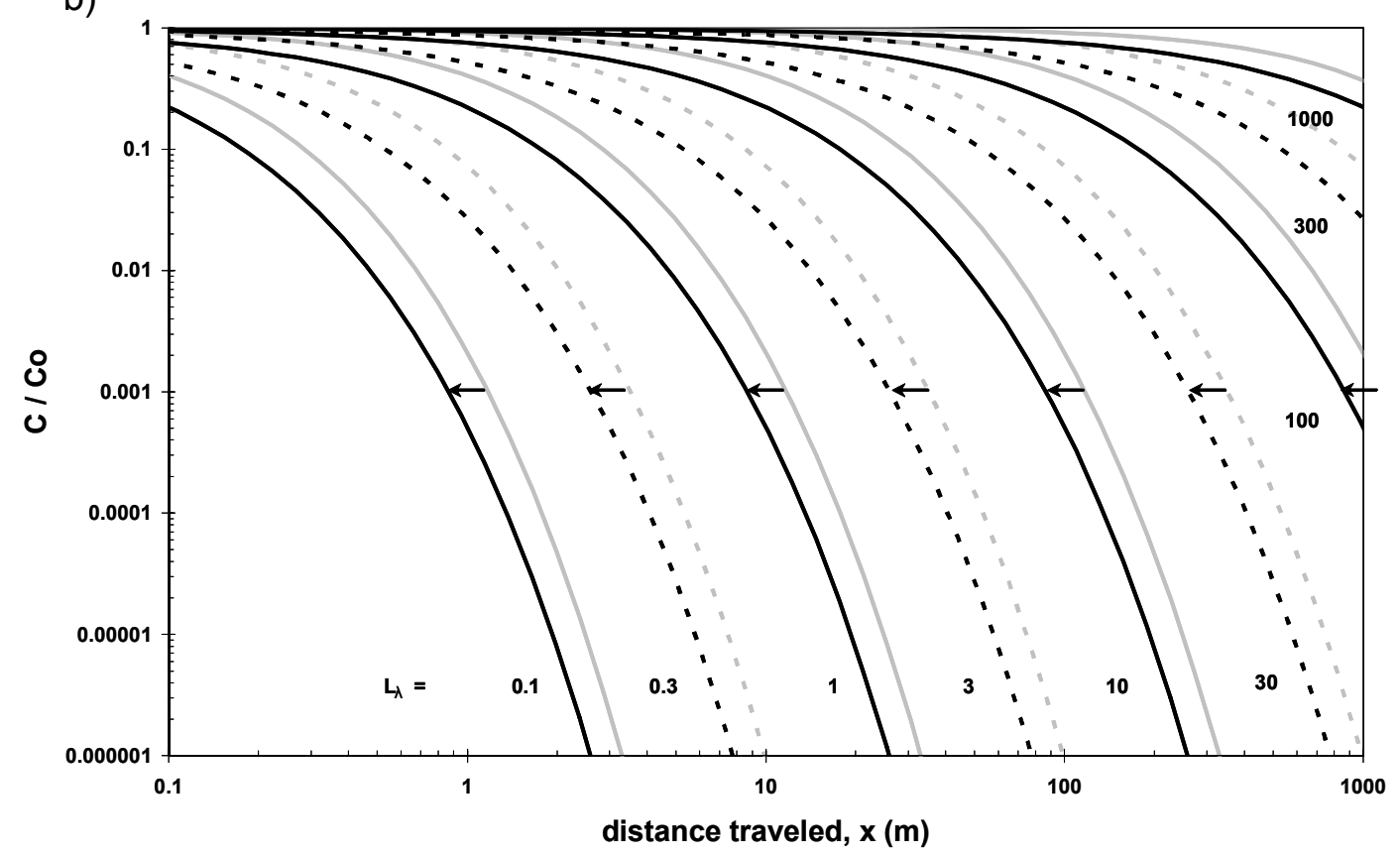

a) results and example case

b) incremental impact of source decay (underlay is steady-state longitudinal dispersion case)

Figure 6-4. Plume size and structure for a transient 1D plume influenced by source decay, degradation, sorption, and longitudinal dispersion 


\section{Case Study 6-4. Determining the Value of Source Removal}

One of the most important decisions at any contaminated site is a determination of "how much" source removal is needed; a key to a responsible decision is the supporting evaluation of the impacts and value of various levels of partial source removal. Source removal is an implicitrequired step in virtually all regulatory paradigms. Overview papers (e.g., Stroo et al., 2003 and Kavanaugh and Rao, 2003) describe this issue and its particular significance for chlorinated organics. Falta et al. (2005a and 2005b) tackled this important challenge using carefully developed and well documented models. Their work generated some notable and thought provoking results.

Several key advances in this work were central to the evaluation, including: 1) development of alternative flux based source model formulations, 2) examination of available data to determine the types of relationships between source removal and flux change, and 3) applying the model to document "effectiveness" under a range of conditions. As shown to the right, the assumed relationship between source removal and concentration/flux was estimated using a power function that is characterized by an exponent $(\Gamma)$, where: $\left(C / C_{0}\right)=\left(M / M_{0}\right)^{\Gamma}$. In this equation, the fraction of the source mass removed is $M / M_{0}$ and the concordant change in flux/concentration is $\mathrm{C} / \mathrm{C}_{0}$. The case of no source removal is in the upper right of the

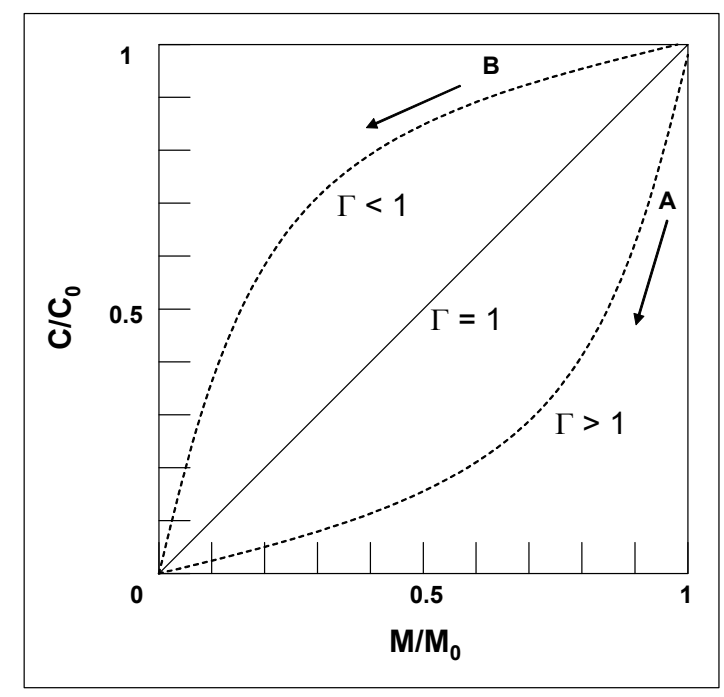
graph and a case of complete source removal at the origin in the lower left. Note that Falta et al. (2005a) cited various case-study examples and the widely varying exponent needed to match the field data. In their follow-on modeling, Falta et al. (2005b) accounted for partial or complete source remediation, either at time zero, or at some later time after the original release. A linked set of solutions was used to compute the time-dependent mass of both parent and daughter contaminants in the plume. A series of examples using different source removal - flux relationships indicated that the benefits of partial source removal vary with site conditions. In general, when $\Gamma>1$, relatively large short-term reductions in the plume concentrations and mass occur, but the source longevity is not strongly affected (see A on graph). Conversely, when $\Gamma<1$, the short-term reductions in the plume concentrations and mass are smaller, but the source longevity can be reduced (B). In either case, the source remediation effort is much more effective if it is undertaken at an early time, before much contaminant mass has entered the plume. If the remediation effort is significantly delayed, the leading parts of the plume are not affected by the source remediation and additional control or remediation of the plume itself may be required to achieve remediation goals. The model runs showed that partial contaminant mass removal from the source zone generally results in significant reductions in near-field plume concentrations and mass. One of the most significant conclusions, however, was that source removal does not dramatically impact the maximum extent of the contaminant plume if very low concentration values are used to define the plume boundaries. Source removal was somewhat effective in reducing the estimated time of remediation, but this benefit was also sensitive to the remediation goals. 


\subsection{SUMMARY OF THE RESULTS FOR 1D CASES}

As formulated, the 1D models are all well behaved. In each case, the plume behavior is predictable based on the reduced variable, $\mathrm{L}_{\lambda}$. Figure 6-5 is a graph of all three $1 \mathrm{D}$ cases in which the travel distance has been normalized to $L_{\lambda}$. Each of the cases is now represented by its type curve. The $\mathrm{x}$-axis is represented by the dimensionless $\mathrm{x} / \mathrm{L}_{\lambda}$. The earlier graphs (Figures 6-1, 6-3, and 6-4) that represent actual physical plume size and structure can be generated from this normalized graph by multiplying by the appropriate site-specific $\mathrm{L}_{\lambda}$. It is clear from these graphs that the most significant factors controlling the plume are those that are embodied in $\mathrm{L}_{\lambda}$. As expected, the most significant factor in spreading the plume is groundwater flow. The factor that has the most potential for contributing to plume stabilization is degradation. If degradation occurs in both the aqueous and sorbed phases, then retardation is also significant. As shown in Figure 6-5, source decay can also play a measurable role in plume stabilization.

In many cases, longitudinal dispersion increased (rather than decreased) the plume extent for any desired concentration reduction endpoint. This was the expected result for the steady-state cases because the infinite inexhaustible source will keep feeding the plume and dispersion will allow some material that would have degraded to reach out further. For the case with relatively strong-rapid source decay $\left(\mathrm{k}_{\mathrm{s}}=\lambda\right)$ longitudinal dispersion sometimes increased and sometimes decreased maximum plume size, but there was a definable region of benefit. The region of benefit can be described based on the desired concentration reduction rather than on flow distance. For our relatively optimistic case of rapid source decay, longitudinal dispersion is beneficial and results in a smaller maximum plume size if the required/desired $\mathrm{C} / \mathrm{Co}$ is $>0.01$ (i.e., one only needs to reduce source concentration by less than a factor of 100 to meet target standards). Longitudinal dispersion caused larger stable plume sizes for all other cases. A general conclusion from the evaluation is that longitudinal dispersion is a relatively weak mechanism and that it will increase plume size unless the source is both weak and short-lived (i.e., decaying).

\subsection{OVERLAY EFFECT OF TRANSVERSE DISPERSION}

\section{See Appendix $B$ for a more detailed presentation of the mathematical formulation used to evaluate transverse dispersion.}

An interesting feature of the 3D analytical solution (Equation 3), is that the transverse dispersion is decoupled and embodied in the terms $\mathrm{f}_{\mathrm{y}}$ and $\mathrm{f}_{\mathrm{z}}$. Importantly these terms do not include time, flow rate, retardation factor, or degradation rate. They are determined entirely by waste site width, and observation position ( $\mathrm{x}, \mathrm{y}$ and $\mathrm{z}$ ). This has two important ramifications. First, the impact of transverse dispersion can be estimated separately from all of the other factors and applied as an overlay. Second, transverse dispersion can result in plume stability for a given $\mathrm{C} / \mathrm{C}_{0}$ even if there are no degradation mechanisms operating for a site. This suggests that an appropriate parametric evaluation of transverse dispersion would be to evaluate the plume centerline value $\mathrm{f}_{\mathrm{y}}$ and/or $\mathrm{f}_{\mathrm{z}}$ as a function of flow distance for bounding cases of "small," "medium" "large" sites and then to overlay the multiplier on the previous 


\section{Plume Structure -- Maximum Predicted Concentration}

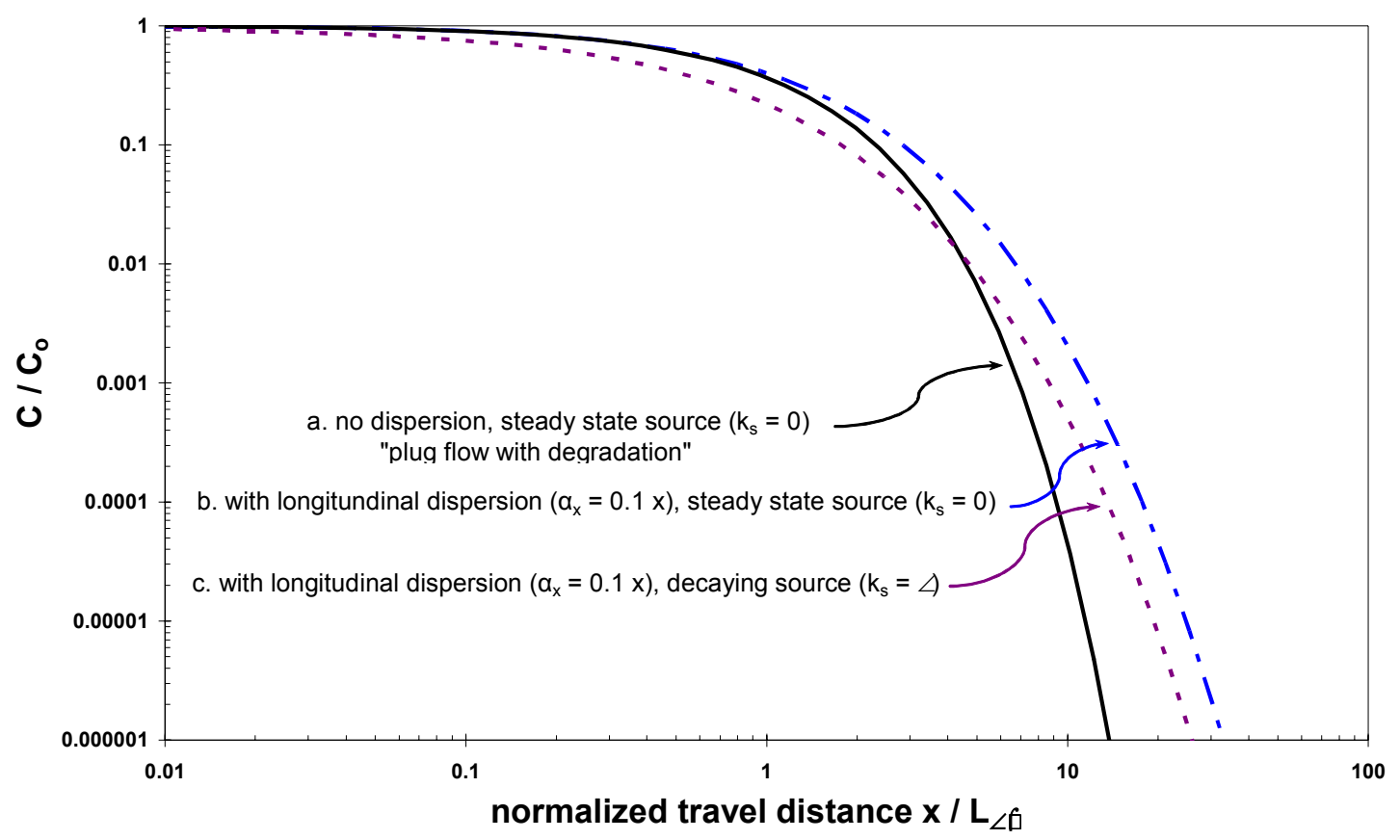

Figure 6-5. Normalized graph showing plume size and structure for a 1D plume under different assumptions about the source and longitudinal dispersion

cases to examine the relative significance of transverse dispersion to the other factors examined previously. For transverse dispersion, the small site would represent the "best case," the medium site the "intermediate case," and the large site the "worst case."

Figure 6-6 depicts the effects of transverse dispersion alone on reducing concentrations and in stabilizing a plume. Specifically, the graph shows the scaled attenuation factor that estimates the impact of transverse dispersion $\left(f_{y} / 2\right)$ versus the flow distance. Figure 6-6 clearly documents the fact that concentration reductions and stable plumes (even for continuous and inexhaustible sources) are predicted even in the absence of degradation mechanisms.

Importantly, the attenuation impacts are much weaker than those embodied in $\mathrm{L}_{\lambda}$. This is clear in the scale of the graph. The y axis, representing the degree of attenuation, spans three orders of magnitude (versus six orders of magnitude on all previous graphs). Also, the $\mathrm{x}$ axis, representing the scale over which the attenuation is occurring, has been extended by three orders of magnitude. Nonetheless, the graph suggests that for small sites where concentration reductions of 0.1 to 0.01 are needed, that a stable/shrinking plume less than $1,000 \mathrm{~m}$ is possible. 


\section{Impact of Lateral Transverse Dispersion on Centerline Concentration}

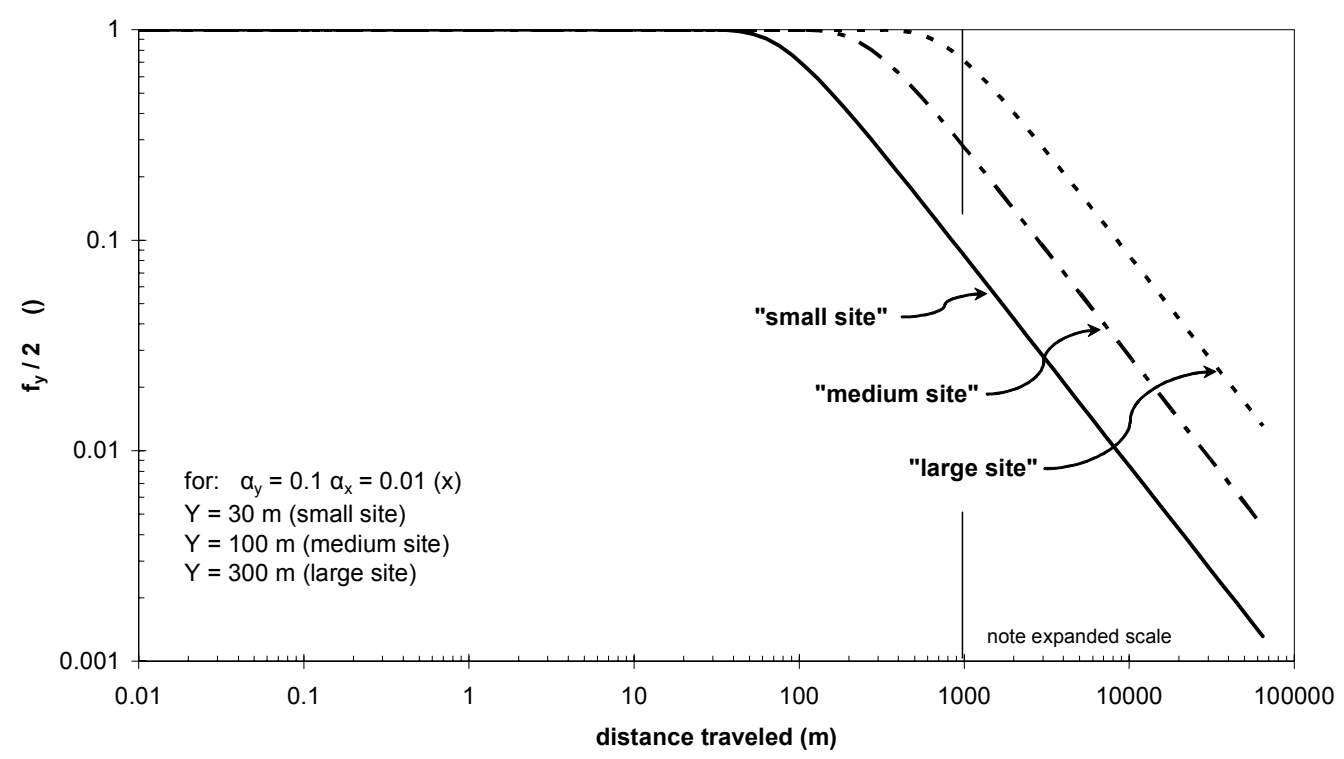

Figure 6-6. General impact of transverse dispersion on centerline plume concentrationscaled attenuation contribution versus flow distance

Figure 6-7 is an example that puts the attenuation resulting from transverse dispersion in context with the previous graphs by overlaying the results on the steady-state $1 \mathrm{D}$ case with longitudinal dispersion. Figure 6-7 represents a small site case.

See Appendix B for the graphs of medium and large site cases.

In all of the graphs, the impact of transverse dispersion increases as a function of flow distance. For small sites, there is no substantive impact of transverse dispersion at distances less than about $100 \mathrm{~m}$. For large sites, there is no substantive impact of transverse dispersion at distances less than about $1000 \mathrm{~m}$. Impacts at medium sites lie between these bounds.

\subsection{SUMMARY OF BOUNDING CASES}

Table 6-4 provides the projected steady-state or maximum plume size for the three bounding cases introduced above. These were:

1) a "best case" which is a small size site with slow groundwater flow velocity, conditions that are ideal for contaminant degradation and a required concentration reduction by a factor of 10 ,

See Case Study 6-5. In this case study, researchers use analytical models to develop an elegant parametric evaluation of MNA and a graphical site evaluation tool. 


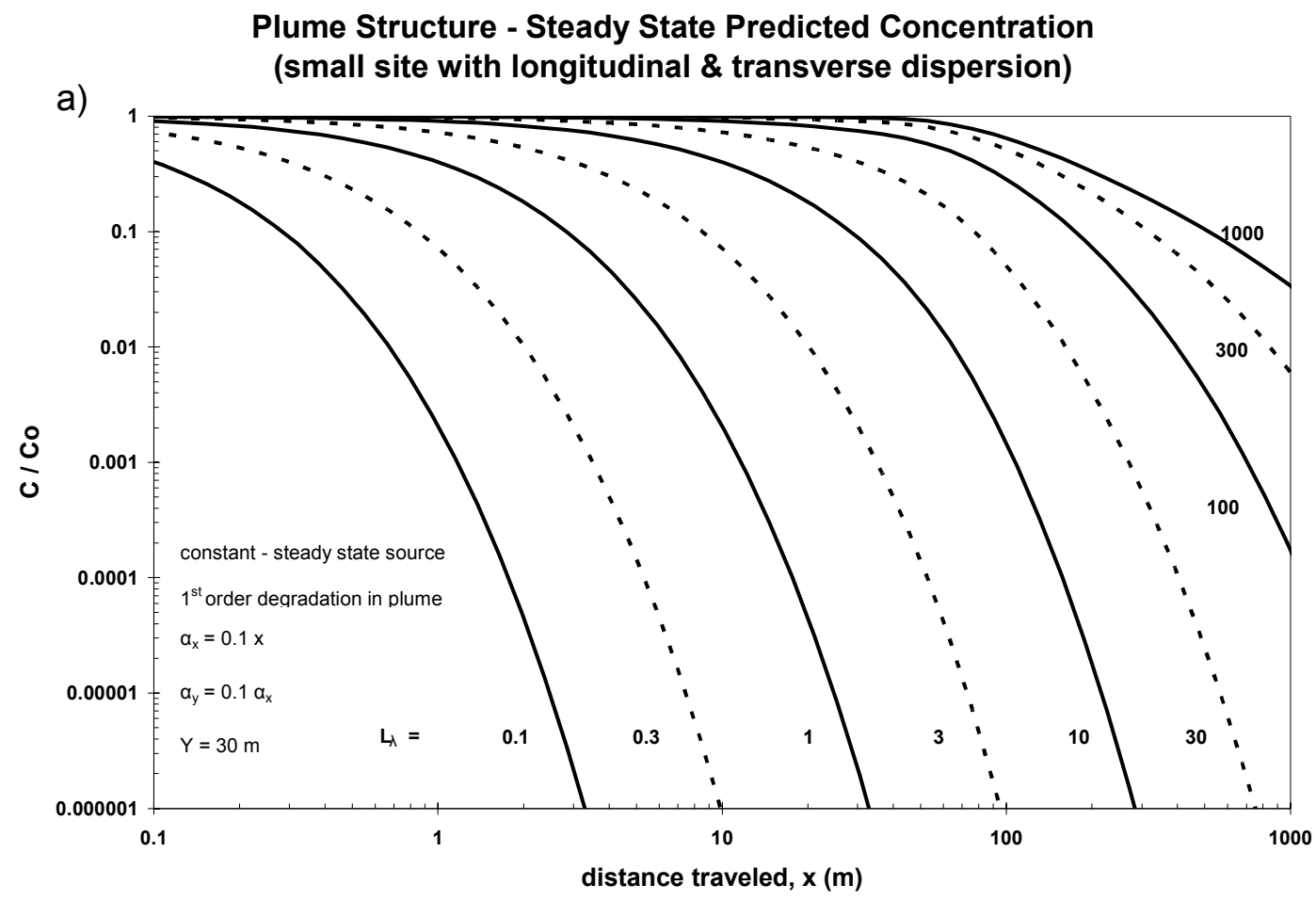

b)

Impact of Transverse Dispersion on Steady State Plume Structure

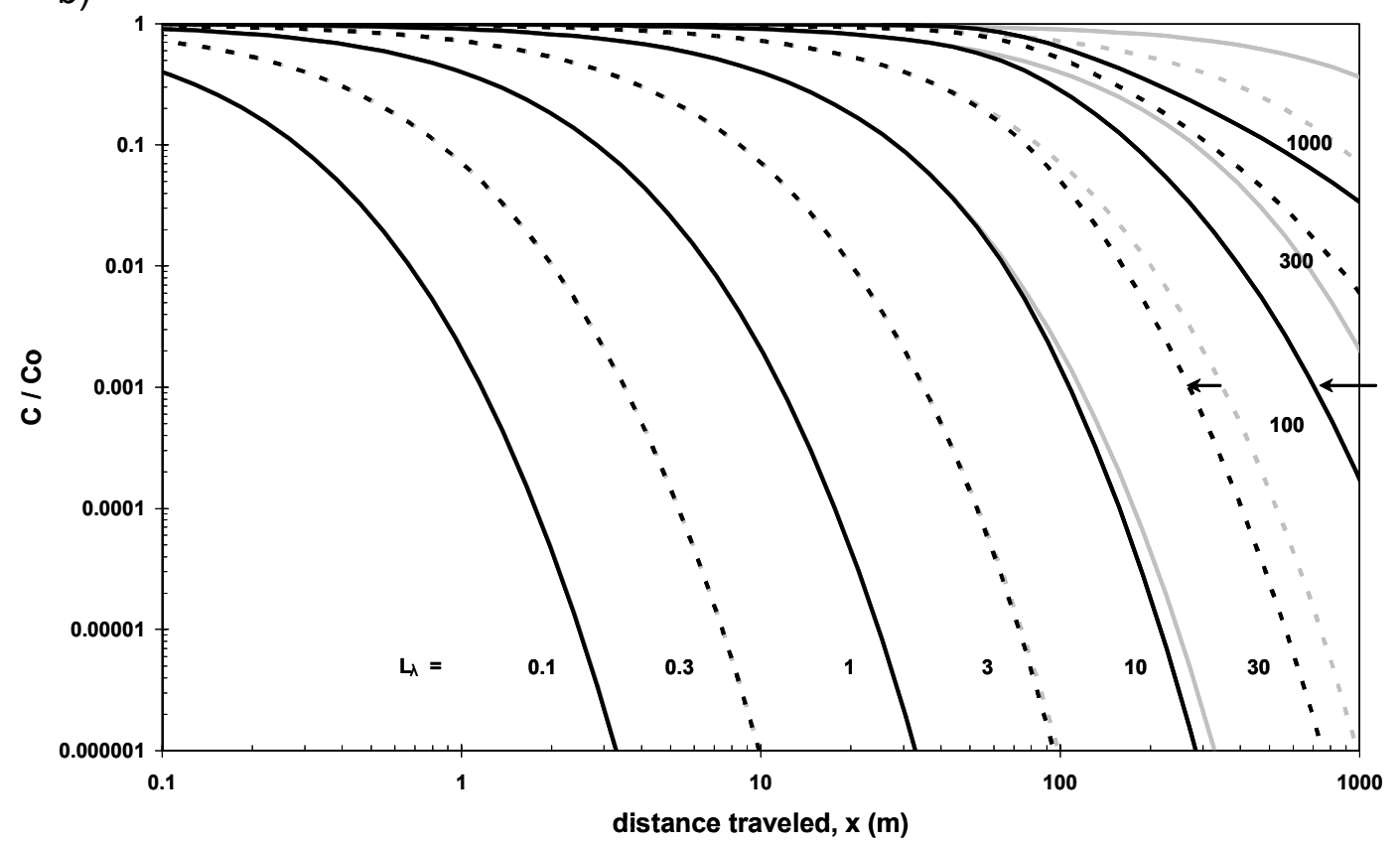

a) general results

b) incremental impact (underlay is 1D steady-state longitudinal dispersion case)

Figure 6-7. Small Site -- Overlay of the attenuation by transverse dispersion for a 2D steady-state plume 


\section{Case Study 6-5. DAFfy Graphs: An Innovative Approach for Modeling}

In the mid-1990s, Johnson and his colleagues developed an elegant and powerful graphical approach to projecting the performance of natural attenuation. The effort, funded by the American Petroleum Institute (API), is a good example of an applied parametric evaluation. The resulting "Graphical approach for determining site specific dilution attenuation factors (DAFs)" distilled complex contaminant source release, fate and transport models into nomographs. These allow simple estimation for contaminant dilution and attenuation under a wide range of site conditions. Because the output of nomographs is a centerline DAF calculated as a function of flow distance, they became known by the shortened name "DAFfy graphs." API published a research bulletin (API 1998), a technical background document and a users guide (Johnson et al., 1998) to facilitate the use of DAFfy graphs. These documents provide detailed background on the models and mathematics used to generate these nomographs, worksheets to assist in organizing the data, example problems, a parameter selection/estimation guide, etc. While not widely used, DAFfy graphs are an interesting and important development. As with other parametric evaluations, the resulting graphs provide insight about the relative significance of the various attenuation mechanisms and provide users with a more robust conceptual understanding of alternative situations. In this case the resulting DAF can be used to forward calculate concentrations in a plume or to back calculate an allowable source concentration from a target receptor concentration. The model development was careful and disciplined; Johnson et al. (1998) included both vadose and submerged sources and minimized some of the "excessive conservatism" that had plagued historical attempts to develop a "one size fits all" generic DAF for regulatory purposes. To use the DAFfy graph approach one simply identifies the source geometry and location, the well screen length of a proposed or existing downgradient well, the aquifer thickness and estimated dispersivity. Additional factors such as degradation rates and time varying sources can also be input. These factors are combined in various ways using a series of graphs to estimate several intermediate variables that are combined to generate the DAF. Importantly, this DAF is theoretically rigorous and is identical to the value that would be calculated if the complicated underlying models were run in full for each case! The following figure is an example of a DAFfy nomograph to calculate " $f$ " ( $f$ is the contribution of degradation to the DAF. Note that the variables in the axis labels are as defined in the original reference):

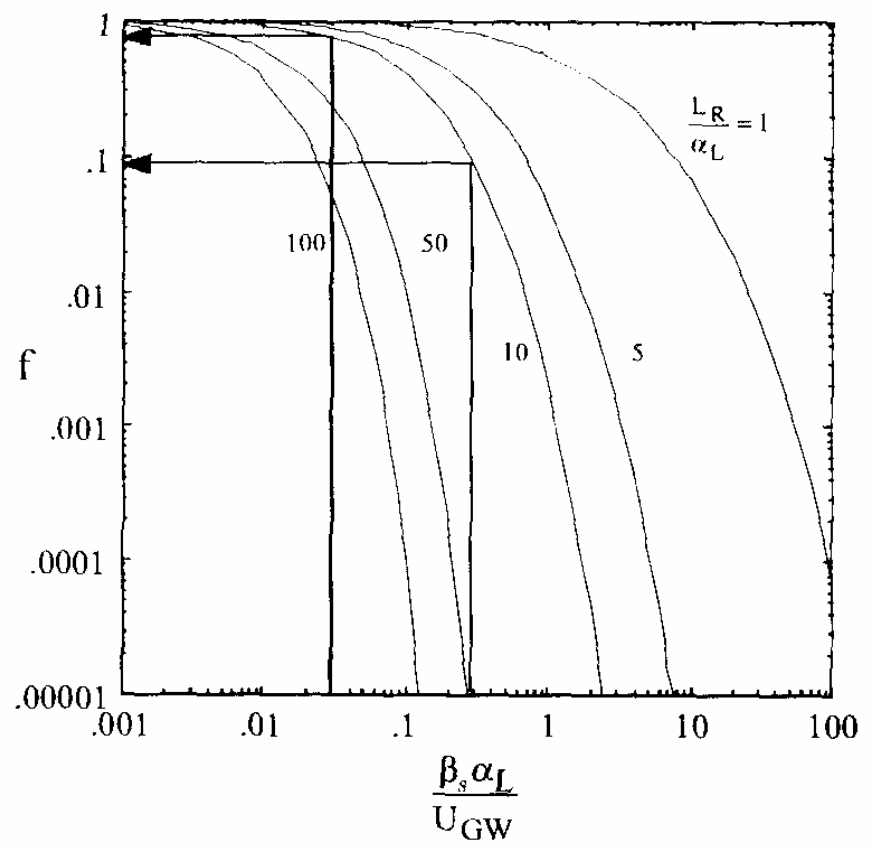


Table 6-4. Projected Steady-State or Maximum Plume Sizes for the Three Bounding Cases

\begin{tabular}{|l|c|c|c|}
\hline & $\begin{array}{c}\text { plug flow model } \\
\text { (steady-state) }\end{array}$ & $\begin{array}{c}\text { dispersion model } \\
\text { (steady-state) }\end{array}$ & $\begin{array}{c}\text { dispersion model with } \\
\text { source decay }\end{array}$ \\
\hline best case* & $0.92 \mathrm{~m}$ & $1.1 \mathrm{~m}$ & $0.70 \mathrm{~m}$ \\
\hline Intermediate case* & $78 \mathrm{~m}$ & $113 \mathrm{~m}$ & $80 \mathrm{~m}$ \\
\hline $\begin{array}{l}\text { worst case -- no } \\
\text { transverse dispersion }\end{array}$ & $6,900 \mathrm{~m}$ & $11,500 \mathrm{~m}$ & $8,700 \mathrm{~m}$ \\
\hline $\begin{array}{l}\text { worst case -- transverse } \\
\text { dispersion }\end{array}$ & N/A & $7,100 \mathrm{~m}$ & $5,400 \mathrm{~m}$ \\
\hline
\end{tabular}

* transverse dispersion does not affect projected plume sizes for the best case and intermediate case

2) an "intermediate case" representing a medium size site with flow and degradation conditions that are representative of many of the sites in the literature and a required concentration reduction by a factor of 100 , and

3) a "worst case" representing a large site with high groundwater flow and minimal degradation and a required concentration reduction factor of 1,000 .

An important feature of Table 6-4 is that transverse dispersion has been factored as appropriate into the plume sizes. The trends in the projections are consistent with the behaviors discussed previously. In this particular analysis, specific projections (distances in $\mathrm{m}$ ) for each bounding case help to provide insights on maximum expected plume sizes for a realistic range of conditions. A striking feature of the results is that the range of possible maximum plume sizes is extremely large - from approximately a meter up to $10 \mathrm{~km}$. The various model results suggest that under best case conditions, maximum expected plume sizes are quite small $(<1 \mathrm{~m})$. While all of the parameters in the best case scenario are clearly within the possible real-world range, such plumes would be unlikely to be identified and regulated. The importance of this case is that it indicates that strongly attenuating plumes are possible under ideal conditions.

The parameter selection process and the plume size projections suggest that real-world regulated plumes are likely to range from approximately the intermediate case to the worst case. The intermediate case results in projected maximum plume sizes of about $100 \mathrm{~m}$. At this projected plume size, transverse dispersion does not substantively contribute to the observed attenuation. This is distinctly different than the behavior for the worst case plumes. Without transverse dispersion, maximum worst case plume size projections are near 10,000 m (circa 6 miles). In this case, however, transverse dispersion becomes a significant and somewhat dominant attenuation mechanism, limiting maximum plume sizes to approximately $6,000 \mathrm{~m}$ (circa 3.5 miles).

Overall, the parametric evaluation supports the general MNA conceptual developments of the 1990s and the idea that destruction processes are often dominant factors at sites with robust natural attenuation. Importantly, however, any destruction mechanism will support attenuation, not just "reductive dechlorination." As an initial step toward examining and including other processes in a mass balance for contaminants, the technical working group 
assembled by DOE developed a set of tables that examined a broad range of attenuation mechanisms.

The content of these tables (e.g., the processes considered, their descriptions, assessment of significance, required conditions, applicable target chemicals, etc.) were developed by consensus. Table 6-5 identifies, describes and evaluates a broad range of attenuation mechanisms - the final column provides a summary assessment of applicability and significance for each process. Table 6-6 is a matrix that describes the potential applicability of each of the processes to a variety of common chlorinated solvent contaminants (and daughter products). The table suggests that there are significant opportunities to improve the technical basis and robustness of MNA/EA remedies. One example is to consider coupling central reductive zones to surrounding or downgradient aerobic zones for a sequential process where the strengths of the various destruction processes are roughly matched to the target contaminants. A second example for some landfill settings might be to consider co-disposed toluene as an aerobic co-metabolite (if oxygen is present) rather than as an electron donor for reductive processes.

These and several other examples are tabulated where processes are opportunistically coupled. Some processes, such as longitudinal dispersion, are included but noted as relatively weak attenuation mechanisms. Finally, some of the mechanisms, such as phytoextraction or volatilization, can substantively contribute to attenuation but may not be dominant or sufficient when considered alone at typical sites. These alternative and substantive mechanisms may be appropriate candidates for adding to screening models for MNA/EA to improve the overall mass balance. 
WSRC-STI-2006-00082, Rev. 0

June 8, 2006

Page 46 of 77

Table 6-5. Consensus Evaluation of Various Attenuation Mechanisms

\begin{tabular}{|c|c|c|c|}
\hline Process & Description & $\begin{array}{c}\text { Requirements, Appropriate Settings I } \\
\text { Conditions, Bottlenecks and } \\
\text { Incompatibilities }\end{array}$ & Coupling Opportunities \\
\hline \multicolumn{4}{|l|}{ Biological Destruction } \\
\hline $\begin{array}{l}\text { Reductive Dechlorination -- } \\
\text { "chlororespiration" }\end{array}$ & $\begin{array}{l}\text { Destruction of a chlorinated organic where the } \\
\text { compound acts as an electron acceptor and is } \\
\text { reduced -- the organism gains energy from the } \\
\text { process. There are several organisms that can do } \\
\text { this, although only Dehalococcoides ethenogenes } \\
\text { has been shown to reduce PCE all the way to ethene } \\
\text { in pure culture. }\end{array}$ & $\begin{array}{l}\text { Occurs in most reducing environments. } \\
\text { Can substantially contribute to degradation } \\
\text { rate. Hydrogen is needed to act as the } \\
\text { electron donor. Reaction rates may slow } \\
\text { down for less chlorinated compounds such } \\
\text { as DCE and VC. Certain compounds inhibit } \\
\text { this process (e.g., CF). }\end{array}$ & $\begin{array}{l}\text { Should be considered as a primary contributor to destruction rate in } \\
\text { anaerobic settings. For in situ deployment (e.g., electron donor injection) } \\
\text { or permeable reactive barrier deployment, enhancement is possible based } \\
\text { on selected amendments and/or configuration. Opportunity for coupling } \\
\text { with abiotic processes and anaerobic oxidation. Can be coupled with } \\
\text { downgradient aerobic processes. }\end{array}$ \\
\hline Anaerobic Oxidation & $\begin{array}{l}\text { Destruction of a chlorinated organic by use of the } \\
\text { compound as an electron donor for metabolism and } \\
\text { resulting in growth and energy. Iron(III), sulfate and } \\
\text { nitrate are example electron acceptors. }\end{array}$ & $\begin{array}{l}\text { Occurs in many anoxic environments (but } \\
\text { requires an alternative electron acceptor). } \\
\text { Can substantially contribute to the } \\
\text { degradation rate of less chlorinated } \\
\text { compounds such as DCE and VC under the } \\
\text { correct conditions. }\end{array}$ & $\begin{array}{l}\text { Should be considered as a contributor to destruction rate for less } \\
\text { chlorinated species such as VC and DCE in anoxic settings that contain } \\
\text { alternative electron acceptors (e.g. sulfate). This process represents an } \\
\text { opportunity for sustainable plume stabilization at sites where the parent } \\
\text { compound (e.g., PCE and TCE) has been degraded and for deployment in } \\
\text { a reaction zone surrounding a central dechlorination zone. Can be } \\
\text { enhanced by substrate injection or deployed in a permeable reactive } \\
\text { barrier. }\end{array}$ \\
\hline $\begin{array}{l}\text { Anaerobic Direct Metabolism -- } \\
\text { "fermentation" }\end{array}$ & $\begin{array}{l}\text { Destruction of chlorinated organics by using them as } \\
\text { terminal electron acceptors for growth and energy } \\
\text { production. In this process the compound serves as } \\
\text { both the electron donor and electron acceptor. Less } \\
\text { common than reductive dechlorination. }\end{array}$ & $\begin{array}{l}\text { Occurs in highly reducing environments. } \\
\text { Can contribute to degradation rate. }\end{array}$ & $\begin{array}{l}\text { May be a potential contributor to destruction rate in anaerobic settings. For } \\
\text { in situ deployment (e.g., electron donor injection) or permeable reactive } \\
\text { barrier deployment, enhancement is possible based on selected } \\
\text { amendments and/or configuration. More selective than reductive } \\
\text { dechlorination to specific compounds (e.g., chloroethenes). }\end{array}$ \\
\hline Anaerobic Co-metabolism & $\begin{array}{l}\text { Destruction of a chlorinated organic where the } \\
\text { compound acts as an electron acceptor and is } \\
\text { reduced. These reactions result from interaction with } \\
\text { reduced compounds in/near the cells or the fortuitous } \\
\text { reaction with an enzyme being formed for another } \\
\text { use in cellular metabolism. The organism does not } \\
\text { gain energy from the process. Example organisms } \\
\text { include methanogens, rare } \mathrm{SO}_{4} \text {-reducers, } \\
\text { homoacetogens and others. }\end{array}$ & $\begin{array}{l}\text { Occurs in most reducing environments but } \\
\text { relatively inefficient. Can contribute to } \\
\text { degradation rate but several other types of } \\
\text { reductive destruction will predominate under } \\
\text { most conditions. Influenced by } \\
\text { geochemistry (e.g., methane levels). In } \\
\text { practice, this is difficult to distinguish from } \\
\text { reductive dechlorination and anaerobic } \\
\text { direct metabolism. }\end{array}$ & $\begin{array}{l}\text { Should be considered as a contributor to destruction rate in anaerobic } \\
\text { settings. For in situ deployment (e.g., electron donor injection) or } \\
\text { permeable reactive barrier deployment. Enhancement is possible based on } \\
\text { selected amendments and/or configuration. }\end{array}$ \\
\hline Aerobic Direct Metabolism & $\begin{array}{l}\text { Destruction of a chlorinated organic by use of the } \\
\text { contaminant as an electron donor for metabolism and } \\
\text { resulting in growth and energy. Oxygen is an } \\
\text { available and efficient electron acceptor in aerobic } \\
\text { conditions. }\end{array}$ & $\begin{array}{l}\text { Requires oxygen and only occurs in aerobic } \\
\text { environments -- but does not destroy highly } \\
\text { chlorinated compounds such as PCE, TCE, } \\
\text { CT and TCA. }\end{array}$ & $\begin{array}{l}\text { Should be considered as a contributor to destruction rate for less } \\
\text { chlorinated species such as VC and DCE in aerobic settings. This process } \\
\text { represents an opportunity for sustainable plume stabilization at sites where } \\
\text { the parents have been degraded and for deployment in a reaction zone } \\
\text { surrounding a central dechlorination zone. }\end{array}$ \\
\hline
\end{tabular}


WSRC-STI-2006-00082, Rev. 0

June 8, 2006

Page 47 of 77

Table 6-5. Consensus Evaluation of Various Attenuation Mechanisms - continued

\begin{tabular}{|c|c|c|c|}
\hline Process & Description & $\begin{array}{l}\text { Requirements, Appropriate Settings / } \\
\text { Conditions, Bottlenecks and } \\
\text { Incompatibilities }\end{array}$ & Coupling Opportunities \\
\hline Aerobic Co-metabolism & $\begin{array}{l}\text { Destruction of a compound where the compound is } \\
\text { fortuitously degraded by an enzyme being formed for } \\
\text { another use in cellular metabolism - for example a } \\
\text { monooxygenase enzyme. }\end{array}$ & $\begin{array}{l}\text { Occurs in an aerobic environment in the } \\
\text { presence of appropriate co-metabolic } \\
\text { substrates (e.g., toluene or methane). This } \\
\text { process is most rapid for the less } \\
\text { chlorinated compounds and can contribute } \\
\text { to degradation rates for all but the most } \\
\text { highly chlorinated compounds (e.g., PCE). }\end{array}$ & $\begin{array}{l}\text { Should be considered as a contributor to destruction rate in aerobic } \\
\text { settings that contain co-metabolic substrates. This process represents an } \\
\text { opportunity for sustainable plume stabilization at sites where the parent } \\
\text { compounds (e.g., PCE) have been degraded. Can occur if a co-metabolic } \\
\text { substrate was co-disposed (e.g., a landfill). Can be deployed in a reaction } \\
\text { zone surrounding a central dechlorination zone. This can be coupled to in } \\
\text { situ (injection based) enhancements or to permeable barriers. }\end{array}$ \\
\hline \multicolumn{4}{|l|}{ Abiotic Destruction } \\
\hline Abiotic & $\begin{array}{l}\text { Destruction of a chlorinated organic where the } \\
\text { contaminant hydrolyzes or acts as an electron } \\
\text { acceptor and is reduced. These reactions may occur } \\
\text { in solution or by the interaction of the contaminant } \\
\text { with reduced minerals (e.g., FeS) on the surfaces of } \\
\text { the aquifer matrix. }\end{array}$ & $\begin{array}{l}\text { Occurs in many environments -- may be } \\
\text { most effective in low oxygen conditions. } \\
\text { Geochemistry (e.g., pH, redox, ionic } \\
\text { strength, etc.) and temperature influence } \\
\text { reaction rates. Mechanisms are highly } \\
\text { compound specific. Promising variants are } \\
\text { coupled (microbially mediated) abiotic } \\
\text { degradation processes. }\end{array}$ & $\begin{array}{l}\text { May be a contributor to destruction rate in anaerobic settings. Often } \\
\text { occurs in combination with biological processes or can be coupled to or } \\
\text { initiated by biological processes. For in situ deployment (e.g., electron } \\
\text { donor injection) or permeable reactive barrier deployment, enhancement is } \\
\text { possible based on selected amendments and/or configuration. }\end{array}$ \\
\hline \multicolumn{4}{|l|}{ Physical } \\
\hline Sorption & $\begin{array}{l}\text { Not destructive. Partitioning from the aqueous phase } \\
\text { into the solid phase. For chlorinated organics, the } \\
\text { amount of partitioning is often assumed to correlate } \\
\text { with the organic carbon content of the solid phase. } \\
\text { Typically, sorption models assume that the } \\
\text { partitioning can be described as a ratio (i.e., a } \\
\text { distribution coefficient or } \mathrm{K}_{d} \text { ) between the solid and } \\
\text { solution phase concentrations or some other similar } \\
\text { simple relationship. }\end{array}$ & $\begin{array}{l}\text { Occurs in all settings. Impacts more highly } \\
\text { chlorinated compounds more than less } \\
\text { chlorinated compounds. For large sources } \\
\text { where no degradation occurs in the sorbed } \\
\text { phase, this factor has minimal impact on } \\
\text { ultimate stable plume size. }\end{array}$ & $\begin{array}{l}\text { May be a contributor to attenuation for small sources (where the source } \\
\text { decays before the maximum plume extent is realized) and if research } \\
\text { indicates that the sorbed contaminant is undergoing destruction by other } \\
\text { mechanisms. Enhancement by increasing organic carbon in the solid } \\
\text { phase may be possible. }\end{array}$ \\
\hline Sorption "irreversible" & $\begin{array}{l}\text { Not destructive. Partitioning from the aqueous phase } \\
\text { into the solid phase. For chlorinated organics, the } \\
\text { amount of partitioning is often assumed to correlate } \\
\text { with the organic carbon content of the solid phase. } \\
\text { "Irreversible" sorption is distinguished from traditional } \\
\text { concepts because it assumes that some of the } \\
\text { material becomes tightly bound and is released } \\
\text { extremely slowly. }\end{array}$ & $\begin{array}{l}\text { May occur in all settings. More data are } \\
\text { needed on the dynamics of this process and } \\
\text { the potential impacts. }\end{array}$ & $\begin{array}{l}\text { May be a contributor to attenuation for small sources (where the source } \\
\text { decays before the maximum plume extent is realized) and if research } \\
\text { indicates that the sorbed phase is undergoing destruction by other } \\
\text { mechanisms. Enhancement by increasing organic carbon in the solid } \\
\text { phase may be possible. }\end{array}$ \\
\hline
\end{tabular}




\section{Table 6-5. Consensus Evaluation of Various Attenuation Mechanisms - continued}

\begin{tabular}{|c|c|c|c|}
\hline Process & Description & $\begin{array}{l}\text { Requirements, Appropriate settings / } \\
\text { Conditions, Bottlenecks and } \\
\text { Incompatibilities }\end{array}$ & Coupling Opportunities \\
\hline Longitudinal Dispersion & $\begin{array}{l}\text { In many cases, longitudinal dispersion increases the } \\
\text { size of a plume -- this is typically not a substantive } \\
\text { attenuation mechanism but can be beneficial under } \\
\text { specific conditions. }\end{array}$ & $\begin{array}{l}\text { Provides benefit under specific conditions } \\
\text { noted under coupling. }\end{array}$ & $\begin{array}{l}\text { In combination with other attenuation mechanisms and under ideal } \\
\text { conditions in which the source flux is falling off rapidly and the amount of } \\
\text { concentration attenuation needed is modest (e.g., }<100 x) \text {, then } \\
\text { longitudinal dispersion can be beneficial and result in a smaller stable } \\
\text { plume size. }\end{array}$ \\
\hline Transverse Dispersion & $\begin{array}{l}\text { Not destructive. Physical spreading of the plume } \\
\text { away from the centerline. }\end{array}$ & $\begin{array}{l}\text { Requires small (size) source area and long } \\
\text { flow distances to be a substantive } \\
\text { attenuation mechanism. Works under all } \\
\text { geochemical conditions. }\end{array}$ & $\begin{array}{l}\text { Weak mechanism that may contribute to attenuation but is not dominant at } \\
\text { most sites. Considering this attenuation mechanism in combination with } \\
\text { other processes may be useful and appropriate for small sites. While } \\
\text { enhancement of this mechanism might be theoretically possible (e.g., using } \\
\text { lateral wells or fracturing), such enhancements are not particularly } \\
\text { promising. }\end{array}$ \\
\hline Volatilization / Phytoextraction & $\begin{array}{l}\text { Typically not destructive. Many chlorinated organics } \\
\text { will volatilize or be extracted by plants and transpired } \\
\text { to the atmosphere where they are subject to } \\
\text { photodegradation. A few plants (e.g., pine and some } \\
\text { hybrid poplars) do stimulate some destruction in the } \\
\text { root zone. }\end{array}$ & $\begin{array}{l}\text { Occurs in the distal portions of plumes near } \\
\text { outcrops or in surface water (seeps, } \\
\text { streams or rivers) after discharge. }\end{array}$ & $\begin{array}{l}\text { Reliable and calculable mechanisms in many settings -- but occurs near } \\
\text { potential exposure point where contaminants are released from the } \\
\text { groundwater system and may not be acceptable to regulators or } \\
\text { stakeholders. }\end{array}$ \\
\hline \multicolumn{4}{|l|}{ Boundary condition } \\
\hline Source Decay/Removal & $\begin{array}{l}\text { Removes contaminant from the system and should } \\
\text { reduce the time period for plume stabilization and } \\
\text { shrinkage. This is an active area of research and } \\
\text { there is potential for the mass balance idea to help } \\
\text { define how much source flux reduction is needed. }\end{array}$ & $\begin{array}{l}\text { Methods include physical removal (e.g., } \\
\text { excavation or soil vapor extraction) and } \\
\text { destruction (e.g., chemical oxidation or } \\
\text { biological reduction). May also result from } \\
\text { depletion over time. }\end{array}$ & $\begin{array}{l}\text { Physical removal often used for small and accessible sources. Source } \\
\text { removal is often used in conjunction with plume interdiction (e.g., pump } \\
\text { and treat) and can be used as a precursor to MNA. May need to consider } \\
\text { long term conditions needed for MNA and couple the decision -- for } \\
\text { example, a long term need for reducing conditions might not be compatible } \\
\text { with chemical oxidation at some sites. Innovations such as barometric } \\
\text { pumping may provide a sustainable EA option for source removal. }\end{array}$ \\
\hline $\begin{array}{l}\text { Water Balance (water and/or } \\
\text { electron acceptor diversion) }\end{array}$ & $\begin{array}{l}\text { Not destructive. Directly modify the contaminant } \\
\text { fluxes and driving forces in a system by reconfiguring } \\
\text { site to bypass water away from a contaminated zone } \\
\text { (either the source zone or the plume). }\end{array}$ & $\begin{array}{l}\text { Applicable to all sites. Many configurations } \\
\text { are possible. In combination with } \\
\text { degradation, this was a dominant parameter } \\
\text { in controlling plume stabilization and } \\
\text { maximum plume size. }\end{array}$ & $\begin{array}{l}\text { May be useful in combination with many other types of remediation and } \\
\text { allow successful EA by altering the mass balance. May have the added } \\
\text { benefit of diverting competing electron acceptors at sites where reductive } \\
\text { processes are desired. }\end{array}$ \\
\hline
\end{tabular}


WSRC-STI-2006-00082, Rev. 0

June 8, 2006

Page 49 of 77

Table 6-6. Applicability of Various Attenuation Mechanisms to Typical Chlorinated Solvents

\begin{tabular}{|c|c|c|c|c|c|c|c|c|c|c|c|c|c|c|}
\hline \multirow[b]{3}{*}{ Process } & & & & & \multicolumn{8}{|c|}{ Example Chlorinated Solvents } & \multirow{2}{*}{\multicolumn{2}{|c|}{$\begin{array}{l}\text { Supporting } \\
\text { Information }\end{array}$}} \\
\hline & \multicolumn{4}{|c|}{ Ethenes } & \multicolumn{4}{|c|}{ Ethanes } & \multicolumn{4}{|c|}{ Methanes } & & \\
\hline & 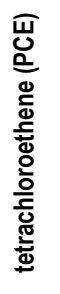 & 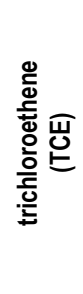 & 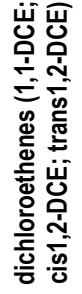 & $\begin{array}{l}\frac{0}{0} \\
\frac{0}{0} \\
\frac{0}{0} \\
\frac{0}{5} \\
\frac{0}{\grave{d}}\end{array}$ & 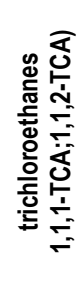 & 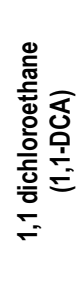 & 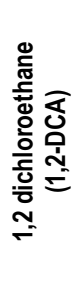 & 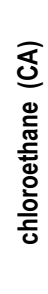 & 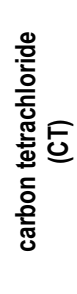 & 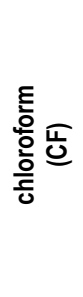 & 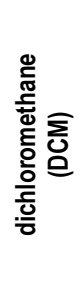 & 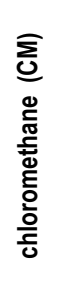 & 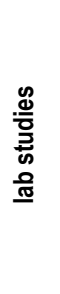 & 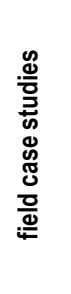 \\
\hline \multicolumn{15}{|l|}{ Biological Degradation } \\
\hline reductive dechlorination & $\bar{Y}$ & $\bar{Y}$ & $\bar{Y}$ & $\bar{L}$ & $\bar{Y}$ & $\bar{Y}$ & $\bar{Y}$ & $\bar{L}$ & $\mathrm{Y}$ & $\bar{Y}$ & $\mathrm{Y}$ & $\bar{L}$ & $\bar{Y}$ & $\mathrm{Y}$ \\
\hline anaerobic oxidation & $\mathrm{N}$ & $\mathrm{N}$ & $\mathrm{L}$ & $\mathrm{Y}$ & $?$ & $?$ & $?$ & $?$ & $\mathrm{~L}$ & $\mathrm{~L}$ & $\mathrm{~L}$ & $?$ & $\mathrm{Y}$ & $\mathrm{L}$ \\
\hline anaerobic direct metabolism & $\mathrm{L}$ & $\mathrm{L}$ & $\mathrm{L}$ & $\mathrm{L}$ & $?$ & $?$ & $\mathrm{~L}$ & $\mathrm{~N}$ & $\mathrm{~N}$ & $\mathrm{~L}$ & $\mathrm{~L}$ & $\mathrm{~L}$ & $\mathrm{~L}$ & $\mathrm{~L}$ \\
\hline aerobic direct metabolism & $\mathrm{N}$ & $\mathrm{N}$ & $Y$ & $\mathrm{Y}$ & $?$ & $?$ & $\mathrm{Y}$ & $\mathrm{Y}$ & $\mathrm{N}$ & $\mathrm{N}$ & $?$ & $\mathrm{Y}$ & $\mathrm{L}$ & $\mathrm{L}$ \\
\hline aerobic cometabolism & $\mathrm{N}$ & $\mathrm{L}$ & $\mathrm{Y}$ & $\mathrm{Y}$ & $\mathrm{L}$ & $\mathrm{Y}$ & $\mathrm{Y}$ & $\mathrm{Y}$ & $\mathrm{N}$ & $\mathrm{Y}$ & $\bar{Y}$ & $Y$ & $Y$ & $\mathrm{Y}$ \\
\hline \multicolumn{15}{|l|}{ Abiotic Degradation } \\
\hline abiotic elimination/hydrolysis & $\mathrm{L}$ & $\mathrm{N}$ & $\mathrm{N}$ & $\mathrm{N}$ & $\mathrm{L}$ & $?$ & $\mathrm{~L}$ & $\mathrm{~L}$ & $\mathrm{~L}$ & $\mathrm{~L}$ & $\mathrm{~L}$ & $\mathrm{~L}$ & $\bar{Y}$ & $\mathrm{~L}$ \\
\hline \multicolumn{15}{|l|}{ Physical - Partitioning and Dispersion } \\
\hline sorption (linear) & $\mathrm{L}$ & $\mathrm{L}$ & $\mathrm{L}$ & $\mathrm{L}$ & $\mathrm{L}$ & $\mathrm{L}$ & $\mathrm{L}$ & $\mathrm{L}$ & $\mathrm{L}$ & $\mathrm{L}$ & $\mathrm{L}$ & $\mathrm{L}$ & $\mathrm{Y}$ & $\mathrm{Y}$ \\
\hline sorption "irreversible" & $?$ & $?$ & $?$ & $?$ & $?$ & $?$ & $?$ & $?$ & $?$ & $?$ & $?$ & $?$ & $\bar{L}$ & $\bar{L}$ \\
\hline longitudinal dispersion & $\mathrm{N}$ & $\mathrm{N}$ & $\mathrm{N}$ & $\mathrm{N}$ & $\mathrm{N}$ & $\mathrm{N}$ & $\mathrm{N}$ & $\mathrm{N}$ & $\mathrm{N}$ & $\mathrm{N}$ & $\mathrm{N}$ & $\mathrm{N}$ & $\mathrm{Y}$ & $?$ \\
\hline transverse dispersion & $\mathrm{L}$ & $\mathrm{L}$ & $\mathrm{L}$ & $\mathrm{L}$ & $\mathrm{L}$ & $\mathrm{L}$ & $\mathrm{L}$ & $\mathrm{L}$ & $\mathrm{L}$ & $\mathrm{L}$ & $\mathrm{L}$ & $\mathrm{L}$ & $\mathrm{Y}$ & $\mathrm{Y}$ \\
\hline volatilization \& phytoextraction & $\mathrm{L}$ & $\mathrm{L}$ & $\mathrm{L}$ & $\mathrm{L}$ & $\mathrm{L}$ & $\mathrm{L}$ & $\mathrm{L}$ & $\mathrm{Y}$ & $\mathrm{L}$ & $\mathrm{L}$ & $\mathrm{Y}$ & $\mathrm{Y}$ & $\mathrm{Y}$ & $\mathrm{Y}$ \\
\hline \multicolumn{15}{|l|}{ Boundary Condition - Source and Hydrology } \\
\hline Source decay/removal & $\mathrm{Y}$ & $\mathrm{Y}$ & $\mathrm{Y}$ & $\mathrm{Y}$ & $\mathrm{Y}$ & $\mathrm{Y}$ & $\mathrm{Y}$ & $\mathrm{Y}$ & $\mathrm{Y}$ & $\mathrm{Y}$ & $\mathrm{Y}$ & $\mathrm{Y}$ & $\mathrm{L}$ & $\mathrm{L}$ \\
\hline water balance (water / $\mathrm{e}-$ acceptor diversion) & $\bar{Y}$ & $\bar{Y}$ & $\bar{Y}$ & $\bar{Y}$ & $\bar{Y}$ & $\bar{Y}$ & $\bar{Y}$ & $\bar{Y}$ & $\bar{Y}$ & $\bar{Y}$ & $\bar{Y}$ & $\bar{Y}$ & $\bar{Y}$ & $\bar{Y}$ \\
\hline
\end{tabular}

$Y$ (yes) means the process has been observed, is applicable, can substantially contribute to MNA/EA, and is likely to occur at a reasonable number of sites.

$\mathrm{L}$ (limited) -- means the process may occur and contribute to MNA/EA but is limited because the rates are slower than those for $Y$ or the process is applicable under a narrower set of environmental conditions.

? (unknown)

$\mathrm{N}$ (no) means that a process is unlikely to contribute substantively to MNA/EA for the subject compound. 
WSRC-STI-2006-00082, Rev. 0 June 8, 2006

Page 50 of 77

This page intentionally left blank. 


\subsection{MASS BALANCE RESEARCH NEEDS AND RESEARCH SUPPORT}

In the simplest sense, a mass balance analysis estimates the contaminant loading and the attenuation capacity of a system. This analysis is normally done using models. Such tools provide a documented approach to link characterization and monitoring data to estimates of performance and provide a tool to examine potential system responses to selected conditions such as transient hydrologic conditions, variations in attenuation processes, and use of remediation techniques. The analysis and predictive functions of modeling are important within the context of applying MNA/EA to 1) help analyze the relative importance of different attenuation and transport processes within a plume, 2) provide timely decision support, for instance, when there is insufficient temporal monitoring data available, 3) evaluate MNA as a remedy to replace existing remedies (e.g., pump and treat) that have perturbed the plume such that data to establish whether the plume is stable will not be available for a long time, 4) evaluate combinations of other actions (e.g., EA), and 5) help interpret monitoring data for transient plumes.

The type of model applied at a specific site is dependent on the site conditions and the intended use of the model. Two basic levels of models are available that are relevant to MNA/EA modeling. Analytical models are capable of solving the general transport equation with specific limitations. Three-dimensional multi-species reactive transport numerical models discretize the transport equation and iteratively solve it within a defined numerical domain. Numerical models allow for more detailed configuration of the model domain to more closely match site features and, therefore, have advantages for some sites. Selection of the appropriate model for a specific site is dependent on the site conditions and configuration-related differences between analytical models and numerical models. Table 7-1 provides a brief overview of considerations for selecting the primary type of modeling analysis based on site properties, in particular based on whether the geochemistry and hydrology of the site readily supports a relatively simple description of attenuation and transport processes or the geochemistry and hydrology is complex.

As an initial activity in DOE's efforts to support MNA/EA, a set of technical targets related to each of the three major topic areas - mass balance, enhancements, and characterization/ monitoring were developed. For mass balance, several targets were identified that merited attention. All of these address how mass balance concepts are used to evaluate an MNA/EA remedy. In the near term, improvement in these elements may enable improved quantification of attenuation processes, better integration of mass balance within the MNA/EA evaluation and implementation process, and expanded application of modeling to support better strategies for characterization and monitoring associated with MNA/EA. As shown, these targets addressed both practical and fundamental needs: 


\section{Table 7-1. Considerations for Selecting Mass Balance Evaluation Approach Based On Site Conditions}

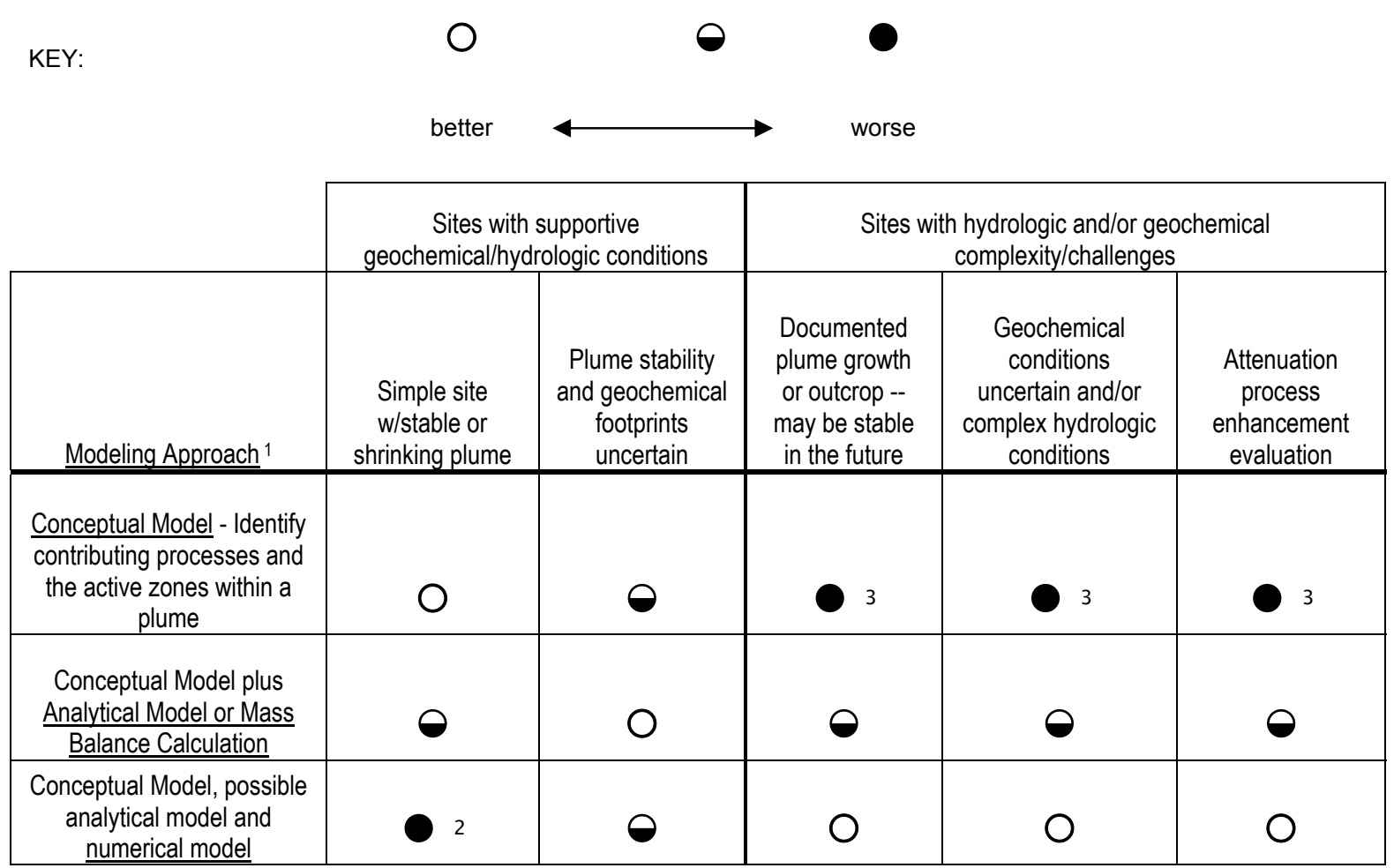

${ }^{1}$ Underlining indicates the primary modeling analysis considered for the table row.

${ }^{2}$ Numerical modeling is not necessarily preferred because costs may not be justifiable for the offsetting benefits in terms of uncertainty reduction, monitoring optimization, etc. However, numerical models may be selected if it is necessary to provide better estimates of timeframes and better assurance of meeting certain types of remediation goals (e.g., concentration targets) than can be obtained with analytical modeling.

${ }^{3}$ Conceptual models are good to use for planning and site management, but may not be suited as primary support for decision-making at complex sites or sites that have high uncertainty because conceptual models do not allow testing of uncertainty and parameter sensitivity and do not strongly support a detailed evaluation of enhancements.

- Define when to use numerical modeling and how to integrate modeling with other elements of MNA evaluation and implementation.

- Improve the description and understanding of the modeling process for MNA.

- Clarify methods to address uncertainty in model inputs and in model interpretation.

- Address numerical model complexity.

- For use of analytical models, address specific limitations of current analytical models.

- Improve BIOCHLOR with expanded reaction forms and variable retardation.

- Improve specific process descriptions for numerical models.

○ Variable sorption phenomena (e.g., a migration resistant fraction)

- Sustainability of reactions

- Abiotic heterogeneous reaction descriptions 
- Substrate and electron acceptor correlations to dechlorination reactions

- Integrate modeling with characterization and monitoring.

$\circ$ Develop new characterization/monitoring techniques that are integrated with model input requirements or interpreted using modeling results.

- Combine modeling with implementation of techniques such as flux monitoring or identify measurement techniques for process parameters such as quantifying time-variant sorption phenomena for a specific site.

In response to these targets, DOE prepared a Federal Request for Information and identified several research projects to address these needs. The resulting descriptive project designations for the funded projects in the three major topic areas are show in Table 7-2. The projects that focus on

See Case Study 7-1. Coupled Models in Action. In this case study, researchers show the value of using a platform to integrate multiple models to address different parts of a problem or plume. or relate to the mass balance needs are noted as primary $(\mathrm{P})$ and secondary (s).

Table 7-2. Selected Projects and Applicability to the Three Major Thrust Areas

Research Study Title

Generating broadly-applicable dehalogenation rate constants

Advancement of nucleic acid based tools for monitoring in situ reductive dechlorination

Oxidative and reductive processes

Use of Electron Shuttles to Biologically Enhance Abiotic Dechlorination

Structural features of VOCs and contaminant solid interactions Irreversible sorption

Scenario based framework for enhanced attenuation/MNA Decision Making

Microsparger for wellhead measurements

Developing an MNA modeling tools based on RT3D

Improving the integration of modeling into MNA: The mass balance utility kit

Strategy and system for long-term monitoring

Innovative oxygen sensor for remote subsurface oxygen measurements

Field testing passive flux meter (PFM) for multiple solute fluxes

Push-Pull tests to determine in situ attenuation capacity at a field site

Enhanced attenuation using bioaugmentation with aerobic bacteria for cis-

DCE

NOTES:

P : Primary link to the identified key concept of the MNA/EA project

s : Secondary link to the identified key concept of the MNA/EA project 
The Mass Balance evaluation generated technical targets for improving quantification of source loading and attenuation processes. One set of targets addresses improvements in applications of modeling that could lead to better strategies for characterization and monitoring MNA/EA. In a manner that is analogous to using both empirical and deterministic methods for characterization and monitoring, coupling of numerical and/or analytical models of processes controlling source loading with models of attenuation process was identified as a strategy for improving mass balance evaluations. Framework for Risk Analysis in Multimedia Environmental Systems (FRAMES) is an example of a model-coupling tool developed for remediation of metals and radionuclides. FRAMES is a platform or operating system that has been used to link a variety of existing source loading and plume process models and, also, link performance evaluations with risk (see, mepas.pnl.gov/FRAMESV1/index.html).

FRAMES was developed by Pacific Northwest National Laboratory. Earlier versions of FRAMES (Versions 1.x) were used mostly to combine exposure assessment models for a variety of environmental media with risk characterization tools for the purpose of evaluating health and ecological risks at federal facilities. Version 2 of the system (FRAMES 2.x) is capable of integrating a much larger variety of models. FRAMES 2.x, designed to integrate codes based on different programming languages, can incorporate models from diverse scientific disciplines. Ho et al. (2002) demonstrated the use of FRAMES to perform a probabilistic, risk-based performance evaluation of a uranium mill tailings disposal cell at the DOE Office of Legacy Management (LM) site near Monticello, Utah. Models were linked in the FRAMES platform to evaluate the soil water balance of the cover, source release from the tailings, vadose-zone transport, saturated-zone transport, and gas release. LM is currently exploring improvements that would better simulate the response of the cover water balance and source loading to ecological change and land management practices (Peterson 2004). Recommendations include integrating an ecological model such as TerreSIM (MFG 2003) or EDYS (Childress et al. 2002), and replacing the existing water balance accounting model with a deterministic unsaturated flow code such as HYDRUS-2D (Simunek et al. 1999) or UNSAT-H (Fayer 2000).

In FRAMES 2.x, every portion of the modeling process, from problem conceptualization to parameterization of model inputs is accomplished quickly within a graphical user interface (GUI). A combination of mouse and keyboard steps lets a user select the environmental media to be simulated, define the processes that will be simulated in those media, draw pertinent data from associated databases, and populate and run multiple models that communicate with each other. Much of a problem's conceptualization is accomplished through "drag-and-drop" exercises with a computer mouse, which leaves clear graphical depictions of the modules involved and the data flow between models.

The FRAMES GUI accesses a variety of tools that not only assist modelers in entering data but also in analyzing simulation results in both graphical and tabular form. A units conversion tool assures users that model parameters selected with one set of units in a model will jibe with those required of a separate model. Decision analysis tools help to visualize simulation results, analyze model sensitivity to different model inputs, and quantify uncertainty in model projections. Data management tools enable FRAMES programmers to develop a full understanding of a modeler's needs and then design efficient data extraction plans.

FRAMES is just one example of a platform where models are coupled - typically these allow some degree of streamlined-graphical data input and data sharing with various output options. Other examples include GMS and a growing variety of commercial products. 


\subsection{MOVING TOWARD A MASS BALANCE APPROACH}

The concept of a mass balance between the loading and attenuation of contaminants in groundwater systems is a powerful framework for conceptualizing monitored natural attenuation (MNA). To be useful in engineering practice, however, it is necessary to quantify this mass balance in ways that facilitate overall site remediation and which are consistent with existing regulatory guidance.

On one hand, mass balance can be approached empirically, where monitoring data from well networks is used to quantify the mass flux of contaminants into a system and the mass removal of contaminants from that system. The empirical approach has the advantage that, when sufficient monitoring data are available, the attenuation capacity can be defined with a large degree of certainty. The disadvantage of the empirical approach is that it is difficult to project how that system will respond to mass removal actions or enhancements of natural attenuation processes. Alternatively, mass balance can be approached deterministically, where models of the physical, chemical, and biological attenuation processes are used to assess contaminant loading and attenuation. The deterministic approach has the advantage that, when sufficient hydrologic, geochemical, and microbiologic data are available, it is possible to project how a system will respond to contaminant removal actions or enhancements of natural attenuation processes. The disadvantage of the deterministic approach is that it is difficult to measure all or most of the relevant hydrologic, geochemical, and biological parameters with any certainty.

The empirical and deterministic approaches to MNA, however, are not mutually exclusive. Our preference is to use a combination of these methods that depends upon the individual challenges presented by a given site. Whenever possible, the empirical approach is used to quantify mass loading and attenuation capacity (contaminant mass/unit time) at particular sites. This is the most effective way to demonstrate the efficiency of ongoing natural attenuation processes in accordance with current regulatory guidance. But in addition, the monitoring well networks needed to apply the empirical approach can also yield estimates of the hydrologic, geochemical, and biological parameters needed to apply deterministic models. Models can then be used to estimate how contaminant behavior will change over time, as contaminant mass is removed, or if attenuation mechanisms are enhanced by engineered methods. The dual use of these empirical and deterministic approaches can help integrate the use of monitored and enhanced natural attenuation for overall site remediation. 
WSRC-STI-2006-00082, Rev. 0 June 8, 2006

Page 56 of 77

This page intentionally left blank. 


\subsection{REFERENCES}

Adams, K. M., K.M. Vangelas, B.B. Looney, F. Chapelle, T. Early, T. Gilmore and C.H. Sink, 2004. Monitored Natural Attenuation and Enhanced Attenuation for Chlorinated Solvent Plumes - It's All About Balance. American Society of Civil Engineers. Proceedings of the World Water and Environmental Resources Congress. Anchorage, Alaska. May 2004.

Al-Suwaiyan, M., 1996, Discussion of "Use of Weighted Least-Squares Method in Evaluation of the Relationship Between Dispersivity and Field Scale," by M. Xu and Y. Eckstein, Ground Water, 34(4):578.

API, 1998. DAFfy Graphs, An Innovative Approach for Modeling the Soil to Water Pathway, in API Soil \& Groundwater Research Bulletin, American Petroleum Institute. Washington D.C., August 1998, No. 7.

Aziz, C. E., C. J. Newell and J. R. Gonzales, 2002. BIOCHLOR Natural Attenuation Decision Support System Users Manual Addendum Verson 2.2, U. S. Environmental Protection Agency, Subsurface Protection and Remediation Division, National Risk Management Research Laboratory, Ada OK March 2002.

Bailey, C. E. and I. W. Marine, 1980. Parametric Study of Geohydrologic Performance Characteristics for Geologic Waste Repositories. E. I. DuPont de Nemours and Company, Savannah River Laboratory, Aiken SC 29808.

Bear, J., 1972. Dynamics of Fluids in Porous Media, Dover Publications, NY.

Bradley, P.M, 2003. History and ecology of chloroethene biodegradation: A Review. Bioremed. J., 7(2):81-109.

Burkholder, H. C. and E. L. J. Rosinger, 1980. A Model for the Transport of Radionuclides and their Decay Products Through Geologic Media. Nuclear Technology 49:150-157.

Butler, E. C. and K. F. Hayes, 2001. Factors Influencing Rates and Products in the Transformation of TCE by Iron Sulfide and Iron Metal. Environ. Sci. Tech., 35: 396.

Chapelle, F.H. and Bradley, P.M. 1998. Selecting remediation goals by assessing the natural attenuation capacity of groundwater systems. Bioremediation Journal, 2: 227-238.

Chapra, S.C. 1996. Surface Water-Quality Monitoring, New York, NY: McGraw Hill.

Childress, W.M., C.L. Coldren, and T.L. McLendon, 2002. Applying a Complex, General Ecosystem Model (EDYS) in Large-Scale Land Management. Ecological Modelling, 153(12):97-108. 
Clement, T.P. 1997. RT3D - A Modular Computer Code for Simulating Reactive Multi-Species Transport in 3-Dimensional Groundwater Aquifers, PNNL-11720, Pacific Northwest National Laboratory, Richland, WA, USA.

Clement, T.P., Y. Sun, B.S. Hooker, and J.N. Petersen. 1998. Modeling Multispecies Reactive Transport in Ground Water. Ground Water Monitoring \& Remediation, 18(2): 79-92.

Clement, T. P., M. J. Truex and P. Lee, 2002. A case study for demonstrating the application of U.S. EPA's monitored natural attenuation screening protocol at a hazardous waste site, Journal of Contaminant Hydrology, 59 pp 133-162.

Clement, T. P., C. D. Johnson, Y. Sun, G. M. Klecka and C. Bartlett, 2000. Natural attenuation of chlorinated ethane compounds: model development and field-scale application at the Dover site, Journal of Contaminant Hydrology, 42 pp 113-140.

Clement, T. P., V. Srinivasan, C. D. Johnson and M. J. Truex, 2006. Development of a new analytical tool for modeling the fate and transport of chlorinated solvent species, U.S. Department of Energy Office of Scientific and Technical Information, Oak Ridge TN.

Domenico, P. A., 1987. An Analytical Model for Multidimensional Transport of Decaying Contaminant Species. J Hydrology, 91:49-58.

Domenico P.A. and Palciauskas, 1982. Ground Water, 20: 301-311.

Einarson, M. D. and D. M. Mackay, 2001. Predicting Impacts of Groundwater Contamination. Environmental Science and Technology 36(3): 66A-73A.

EPA, 1986, Background Document for the Ground-Water Screening Procedure to Support 40 CFR Part 269 - Land Disposal. EPA/530-SW-86-047, U.S. Environmental Protection Agency, Washington DC, January 1986.

EPA, 1998. Technical protocol for evaluating natural attenuation of chlorinated solvents in ground water, EPA/600/R-98/128, Environmental Protection Agency, Office of Research and Development, Washington, D.C.

EPA, 1999. Use of Monitored Natural Attenuation at Superfund, RCRA Corrective Action, and Underground Storage Tank Sites, Final, Office of Solid Waste and Emergency Response. April 21. Directive Number 9200.4-17P, U.S. Environmental Protection Agency, Washington, D.C.

EPA, 2000. BIOCHLOR Natural Attenuation Decision Support System Users Manual Verson 1.0, EPA/600/R-00/008, U. S. Environmental Protection Agency, Office of Research and Development, Wahington DC. January, 2000.

Falta, R.W., P.S.C. Rao, and N. Basu 2005a. Assessing the Impacts of Partial Mass Depletion in DNAPL Source Zones: I. Analytical Modeling of Source Strength Functions and Plume Response, Journal of Contaminant Hydrology, Vol. 78, 259-280. 
Falta, R.W., N. Basu, and P.S.C. Rao, 2005b. Assessing the Impacts of Partial Mass Depletion in DNAPL Source Zones: II. Coupling source strength functions to plume evolution, Journal of Contaminant Hydrology, Vol. 79, 45-66.

Fayer, M.J., 2000. UNSAT-H Version 3.0: Unsaturated Soil Water and Heat Flow Model, Theory, User Manual, and Examples. Pacific Northwest National Laboratory, PNNL-13249.

Ferrey, M. L., R. T. Wilkin, R. G. Ford and J. T. Wilson, 2004. Nonbiological Removal of cisDichloroethylene and 1,1-Dichloroethylene in Aquifer Sediment Containing Magnetite. Environmental Science \& Technology 38(6):1746-1752.

Gelhar, L. W., C. Welty and K. R. Rehfeldt, 1992. A Critical Review of Data on Field Scale Dispersion in Aquifers, Water Resources Res. 28:1955-1974.

Ho, C.K., B.W. Arnold, J.R. Cochran, and R.Y. Taira, 2002. Development of a Risk-Based Probabilistic Performance-Assessment Method for Long-Term Cover System $-2^{\text {nd }}$ Edition. SAND2002-2131, Sandia National Laboratories, Albuquerque, NM,

Johnson, P.C., R.J. Charbeneau, D. Abranovic and T. Hemstreet. 1998. Graphical Approach for Determining Site-Specfic Dilution-Attenuation Factors (DAFs) Technical Background Document. And Users Guide, API Publication 4659. American Petroleum Institute. Washington D.C.

Kavanaugh, M. C. and P. Suresh C. Rao, 2003. The DNAPL Remediation Challenge: Is There a Case for Source Depletion?, EPA/600/R-03/143, U. S. Environmental Protection Agency, National Risk Management Research Laboratory Office of Research and Development, Cincinnati, $\mathrm{OH}$.

McGuire, T. M., C. J. Newell, B. B. Looney and K. M. Vangelas., 2004. Historical and Retrospective Survey of Monitored Natural Attenuation: A Line of Inquiry Supporting Monitored Natural Attenuation and Enhanced Passive Remediation of Chlorinated Solvents, WSRC-TR-2003-00333, Savannah River National Laboratory, Aiken SC, available through www.osti.gov .

MFG, Inc., 2003. TerreSIM, Terrestial Ecosystem Simulation Model, Version 1.0, Users Guide.

MN-PCA, 1999. Guidelines: Natural Attenuation of Chlorinated Solvents in Groundwater. Minnesota Pollution Control Agency, Site Remediation Section, St Paul MN.

Newell, CJ. and JA. Connor, 1998. Characteristics of Dissolved Petroleum Hydrocarbon Plumes, Version 1.1, American Petroleum Institute.

Newell, C.J., J.A. Conner, 1998. Characteristics of Dissolved Petroleum Hydrocarbon Plumes, Version 1.1, American Petroleum Institute.

Newell, C. J., S. S. Rifai, J. T. Wilson, J. A. Connor, J. A. Aziz and M. P. Suarez, , 2002. Calculation and Use of First Order Rate Constants for Monitored Natural Attenuation Studies. 
EPA/540/S-02/500, U. S. Environmental Protection Agency, Subsurface Protection and Remediation Division, National Risk Management Research Laboratory, Ada OK March 2002.

Newell, C.J. and D.T. Adamson, 2005. Planning-level source decay models to evaluate impact of source depletion on remediation time frame. Remediation, 15(2):27-47.

Peterson, D.M., 2004. An Ecohydrological Model for Assessing the Performance of Disposal Cell Covers. Environmental Sciences Laboratory, U.S. Department of Energy, Grand Junction, $\mathrm{CO}$.

Pickens, J. F. and G. E. Grisak, 1981. Scale Dependent Dispersion in a Stratified Granular Aquifer. Water Resources Res. 17:1191-1211.

Rogers, V. and C. Hung, 1987. PATHRAE-EPA: A Low Level Radioactive Waste Environmental Transport and Risk Assessment Code, Methodology and Users Manual, EPA 520/1-87-028, U.S. Environmental Protection Agency Office of Radiation Programs, Washington DC.

Secrist III, P.M. 2002. A Numerical Model (SEAM3D) to Assess the Biotransformation of Chlorinated Ethenes at a TCE/BTEX Contaminated Site. Masters Thesis, Environmental Engineering, Virginia Tech, Blacksburg VA.

Semprini, L., P. K. Kitanidis, D. H. Kampbell and J. T. Wilson, 1995. Anaerobic transformation of chlorinated aliphatic hydrocarbons in a sand aquifer based on spatial chemical distributions, Water Resources Research, Vol. 31 No. 4, pp 1051-1062.

Simunek, J., M. Sejna, and M. Th. Van Genuchten, 1999. The HYDRUS-2D Software Package for Simulating Two-Dimensional Movement of Water, Heat, and Multiple Solutes in VariablySaturated Media, Version 2.0. U.S. Salinity Laboratory, USDA, Riverside, California.

Stroo, H.F., Unger, M., Ward, C.H., Kavanaugh, M.C., Vogel, C., Leeson, A., Marqusee, J.A., Smith, B.P., 2003. Remediating chlorinated solvent source zones. Environ. Sci. Technol. 37 (11), 224A-230A.

Suarez, M.P. and Rifai, H.S., 2002. Evaluation of BTEX remediation by natural attenuation at a coastal facility. Ground Water Monitoring \& Remediation 22(1):62-75.

Van Genuchten, M. Th. and W. J. Alvez, 1982. Analytical Solutions of the One-Dimensional Convective-Dispersive Solute Transport Equation, Technical Bulletin No. 1661, U. S. Department of Agriculture, Washington DC.

WA-DE, 2004. Guidance on Remediation of Petroleum-Contaminated Ground Water by Natural Attenuation. Publication No. 03-09-101 A, Washington State Department of Ecology Toxics Cleanup Program, Olympia WA. 
Wexler, E. J., 1992. Analytical Solutions for one-, two-, and three-dimensional solute transport in ground-water systems with uniform flow, Techniques of Water-Resources Investigations of the United States Geological Survey, Book 3, Chapter B7, U>S. Geological Survey, Washington DC.

WI-DNR, 2003. Understanding Chlorinated Solvent Behavior in Groundwater: Investigation, Assessment and Limitations of MNA, Report No. RR-699, Wisconsin Department of Natural Resources Remediation and Redevelopment Program, Madison WI.

Wiedemeier, T.W., H. S. Rifai, C. J. Newell, and J. W. Wilson, 1999. Natural Attenuation of Fuels and Chlorinated Solvents. John Wiley and Sons, NY.

Wilson, J. T. and P. J. Miller, 1978. Two dimensional plume in uniform ground-water flow, ASCE, J. Hydraulics Division, Volume 104 No. HY4, pp 503-514.

$\mathrm{Xu}, \mathrm{M}$. and Y. Eckstein, 1995. Use of Weighted Least Squares Method in Evaluation of the Relationship Between Dispersivity and Scale, Ground Water 33:905-908. 
WSRC-STI-2006-00082, Rev. 0 June 8, 2006

Page 62 of 77

This page intentionally left blank. 


\section{APPENDIX A. SUPPLEMENTAL INFORMATION - DERIVATION OF TRANSPORT EQUATION}

Note the information in this appendix incorporates a significant amount of information from the peer reviewers. In particular, we would like to acknowledge Ron Falta who provided detailed and specific suggestions that are incorporated into the text and equations below.

\section{Addendum for Section 4.1}

Consider a control volume of dimensions $\Delta x, \Delta y, \Delta z$. Groundwater, with a darcy velocity of $\mathrm{v}_{\mathrm{d}}$ flows in the $\mathrm{x}$-direction as shown:

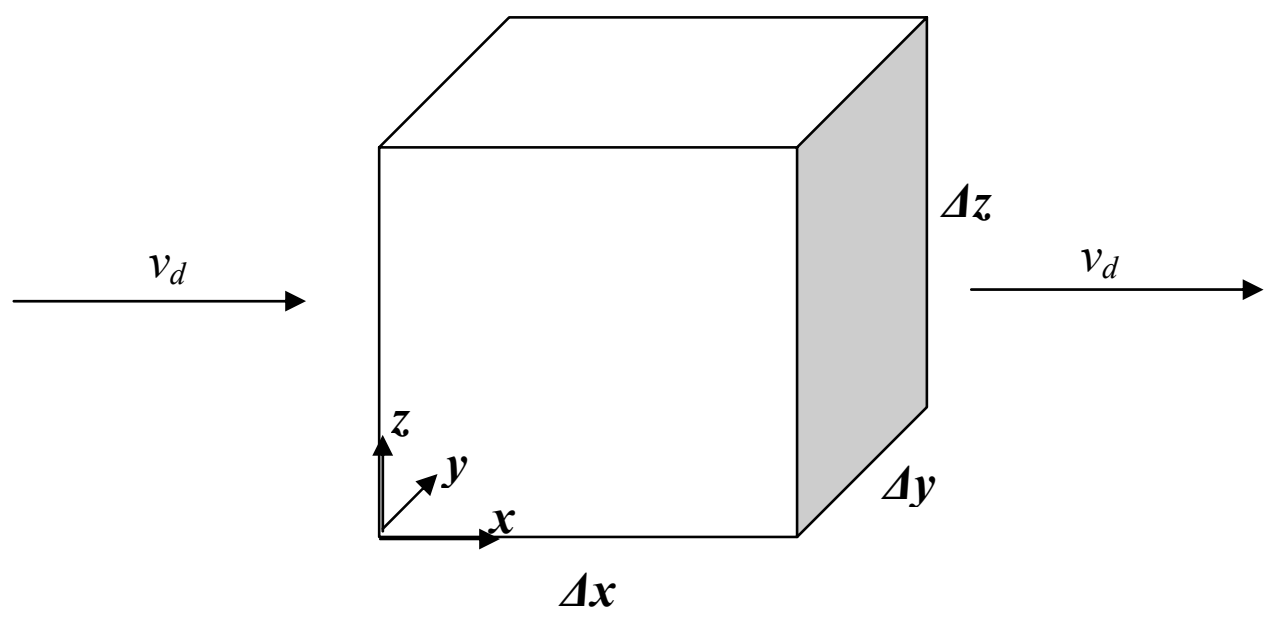

Figure 4-2. Typical "control volume" used to develop mass balance equations for contaminant plumes

The transport equation is based on conservation of (chemical) mass. For any control volume, this law can be stated as:

Rate of mass accumulation inside volume = Rate of mass entering volume - Rate of mass leaving volume - Rate of mass destruction inside volume

The rate of mass accumulation inside the volume accounts for the time rate of change in the chemical mass in both the dissolved and adsorbed phases. Assuming linear equilibrium partitioning, the adsorbed mass fraction is related to the dissolved mass concentration by

$$
X_{\text {solid }}=K_{D} C
$$

Where $\mathrm{K}_{\mathrm{D}}$ is the soil-water distribution coefficient for the chemical in the aquifer. To convert this adsorbed mass fraction to a mass concentration, the soil dry bulk density, $\rho_{b}$ is used: 


$$
C_{\text {solid }}=X_{\text {solid }} \rho_{b}
$$

Then the rate of mass accumulation inside the volume is:

$$
\text { rate of accumulation }=\text { volume } \cdot \frac{\partial}{\partial t}\left(\phi C+\rho_{b} K_{D} C\right)=\Delta x \Delta y \Delta z\left(\phi+\rho_{b} K_{D}\right) \frac{\partial C}{\partial t}
$$

where $\phi$ is the porosity. The term $\phi \mathrm{C}$ accounts for the chemical mass in the aqueous phase, while the term $\rho_{b} K_{D} C$ accounts for the mass that is adsorbed.

Chemical mass can enter or leave the control volume by either advection or dispersion. In most situations, the advective flux in the $\mathrm{x}$-direction is much larger than the dispersive flux in that direction. Because it was assumed that the groundwater velocity was aligned with the $\mathrm{x}$ axis, there are no advective fluxes in the $\mathrm{y}$ and $\mathrm{z}$ directions, so these mass fluxes consist only of dispersive fluxes.

The advective flux is simply the aqueous chemical concentration multiplied by the darcy velocity

$$
\text { advective flux }=v_{d} C
$$

The advective mass flows entering the control volume include the volume surface area perpendicular to the flow, and they must account for the fact that the concentration varies in space. For this reason, it becomes important to specify where $\mathrm{C}$ is evaluated. By convention, we could assume that all mass flows are entering the control volume at the $\mathrm{x}, \mathrm{y}$, and $\mathrm{z}$ faces, and all of the mass flows leave from the control volume faces located at $x+\Delta x, y+\Delta y$, and $\mathrm{z}+\Delta \mathrm{z}$. In other words, mass fluxes are positive in the positive coordinate directions. Note that this choice of coordinate directions was arbitrary, and any convention can be used provided that it is consistent throughout the derivation. With the sign convention given above, the advective mass flow entering the volume is

$$
\text { advective flow entering }=\Delta y \Delta z v_{d} C(x)
$$

Similarly the advective mass flow leaving the volume is

$$
\text { advective flow leaving }=\Delta y \Delta z v_{d} C(x+\Delta x)
$$

where $C(x+\Delta x)$ means that the concentration is evaluated at $x+\Delta x$.

Dispersion can be considered in using a similar conceptual process (accounting for the alignment of the grid and potential inputs and losses in all of the vector directions). Dispersive fluxes are calculated by assuming a Fickian diffusion model that depends on a velocity dependent dispersion coefficient, and the concentration gradient. The dispersive fluxes account for the porosity, and are written as 


$$
\text { dispersive flux in direction } j=-\phi D_{j} \frac{\partial C}{\partial j}
$$

where $D_{j}=\alpha_{j} V_{d} / \Phi$, and $\alpha_{j}$ is the dispersivity in direction $j$. Note that this formulation is only correct if the flow direction is perfectly aligned with the coordinate axes; if this is not the case, the dispersive fluxes are much more complex.

The dispersive mass flows in each direction are the product of the mass fluxes with the appropriate surface areas, using the appropriate dispersivities (but the same velocity). Therefore:

$$
\begin{gathered}
x-\text { dispersive flow entering }=-\Delta y \Delta z \phi D_{l} \frac{\partial C(x)}{\partial x} \\
x-\text { dispersive flow leaving }=-\Delta y \Delta z \phi D_{l} \frac{\partial C(x+\Delta x)}{\partial x} \\
y-\text { dispersive flow entering }=-\Delta x \Delta z \phi D_{t} \frac{\partial C(y)}{\partial y} \\
y-\text { dispersive flow leaving }=-\Delta x \Delta z \phi D_{t} \frac{\partial C(y+\Delta y)}{\partial y} \\
z-\text { dispersive flow entering }=-\Delta x \Delta y \phi D_{v} \frac{\partial C(z)}{\partial z} \\
z-\text { dispersive flow leaving }=-\Delta x \Delta y \phi D_{v} \frac{\partial C(z+\Delta z)}{\partial z}
\end{gathered}
$$

The final term in the mass balance equation accounts for decay (or formation) of the chemical in the volume. There are many different kinetic expressions that describe the degradation of contaminants in natural systems. The mechanisms of degradation include abiotic processes such as hydrolysis or redox reactions with solid minerals, and biological reactions. Biological reactions are often described using enzyme saturation kinetics according to the MichaelisMenton equation while abiotic reactions can follow many different kinetic models. At low to moderate concentrations, however, many processes are reasonably well described by pseudofirst order kinetics and many models use such kinetics as the basis of predictions. If the chemical only decays in the aqueous phase, then the rate of chemical destruction in the volume is 
where $\lambda$ is the first-order decay rate. Note that in the case of chlorinated solvents, a "daughter contaminant" may be formed by the breakdown of a "parent compound" and models that account for formation of a contaminant in such sequential pathways are available.

Substituting the terms into the mass balance equation, and dividing both sides by the volume and porosity, gives:

$$
\begin{array}{r}
\left(1+\frac{\rho_{b} K_{d}}{\phi}\right) \frac{\partial C}{\partial t}=\frac{V_{w}}{\phi}\left(\frac{C(x)-C(x+\Delta x)}{\Delta x}\right)-D_{i}\left(\frac{\frac{\partial C(x)}{\partial x}-\frac{\partial C(x+\Delta x)}{\partial x}}{\Delta x}\right)-D_{v}\left(\frac{\frac{\partial C(z)}{\partial z}-\frac{\partial C(z+\Delta z)}{\partial z}}{\Delta z}\right)-\lambda C \\
-D_{t}\left(\frac{\left.\frac{\partial C(y)}{\partial y}-\frac{\partial C(y+\Delta y)}{\partial y}\right)}{\Delta y}\right)
\end{array}
$$

Defining a retardation factor as

$$
R=\frac{\text { total mass }}{\text { aqueous mass }}=\frac{\phi C+\rho_{b} K_{D} C}{\phi C}=1+\frac{\rho_{b} K_{D}}{\phi}
$$

using the calculus definition of a partial derivative:

$$
\frac{\partial f}{\partial x}=\lim _{\Delta x \rightarrow 0}\left(\frac{f(x+\Delta x)-f(x)}{\Delta x}\right)
$$

and taking the limit of equation 14 as $\Delta x, \Delta y$, and $\Delta \mathrm{z}$ go to zero gives the classical partial differential equation (PDE) for chemical transport in groundwater:

$$
R \frac{\partial C}{\partial t}=-v_{s} \frac{\partial C}{\partial x}+D_{x} \frac{\partial^{2} C}{\partial x^{2}}+D_{y} \frac{\partial^{2} C}{\partial y^{2}}+D_{z} \frac{\partial^{2} C}{\partial_{z}^{2}}-\lambda C
$$

where $\mathrm{v}_{\mathrm{s}}$ is the seepage velocity or pore velocity (the darcy velocity divided by the porosity). Equation 18 is called the governing PDE for this problem, but it must still be solved either analytically or numerically to get values of $\mathrm{C}(\mathrm{x}, \mathrm{y}, \mathrm{z}, \mathrm{t})$. The governing PDE is always subject to boundary and initial conditions. Typically, it is assumed that the problem domain is initially free of chemical, so the normal initial condition is 


$$
t=0, C=0
$$

Boundary conditions can be quite complex in numerical models, but analytical models usually assume a mathematically infinite domain in the $\mathrm{y}$ and $\mathrm{z}$ directions, and a semi-infinite domain in the $\mathrm{x}$-direction, starting at zero. The boundary condition at $\mathrm{x}=0$ is critical in the model, because this is typically where chemical mass discharge from the source zone is allowed to enter the plume model. For example in BIOCHLOR, a rectangular $\mathrm{Y}$ by $\mathrm{Z}$ domain at $\mathrm{x}=0$ is defined so that

$$
\begin{aligned}
& x=0,-\frac{Y}{2} \leq y \leq \frac{Y}{2}, 0 \leq z \leq Z, \quad C=C_{0} \text { or } C_{0} e^{-k t} \\
& C=0 \text { elsewhere }
\end{aligned}
$$

This is known as a "specified concentration" boundary condition.

\section{Return to Section 4.1}


WSRC-STI-2006-00082, Rev. 0 June 8, 2006

Page 68 of 77

This page intentionally left blank. 


\section{APPENDIX B. SUPPLEMENTAL MODELING INFORMATION FOR PARAMETRIC EVALUATION}

Addendum for Section 6.2 - A more detailed discussion of Equation 3

The full solution relatively straightforward:

$$
C_{(x, y, z, t)}=C_{0} e^{-k_{s} t} \frac{f_{x}}{2} \frac{f_{y}}{2} \frac{f_{z}}{2} \quad \text { Equation } 3
$$

The first term of this equation is the initial source concentration with an exponential to describe a decreasing source over time. If the source is constant and inexhaustible, then $\mathrm{k}_{\mathrm{s}}$ is set to 0 and the equation becomes identical to Domenico (1987). Each of the following terms describes the attenuation due to dispersion and degradation. The primary term, $\mathrm{f}_{\mathrm{x}}$, accounts for advection, longitudinal dispersion and first order decay. The remaining terms account for dispersion only in the lateral $\mathrm{x}$ and $\mathrm{y}$ directions, respectively.

where:

$$
\begin{gathered}
f_{x}=\exp \left[\frac{x\left(1-\sqrt{1+4 \alpha_{x}\left(\lambda-k_{s}\right) / v_{c}}\right)}{2 \alpha_{x}}\right] * \operatorname{erfc}\left[\frac{x-v_{c} t\left(1-\sqrt{1+4 \alpha_{x}\left(\lambda-k_{s}\right) / v_{c}}\right)}{2 \sqrt{\alpha_{x} v_{c} t}}\right]+ \\
\exp \left[\frac{x\left(1+\sqrt{1+4 \alpha_{x}\left(\lambda-k_{s}\right) / v_{c}}\right)}{2 \alpha_{x}}\right] * \operatorname{erfc}\left[\frac{x+v_{c} t\left(1-\sqrt{1+4 \alpha_{x}\left(\lambda-k_{s}\right) / v_{c}}\right)}{2 \sqrt{\alpha_{x} v_{c} t}}\right] \\
f_{y}=\operatorname{erf}\left[\frac{y+Y / 2}{2 \sqrt{\alpha_{y} x}}\right]-\operatorname{erf}\left[\frac{y-Y / 2}{2 \sqrt{\alpha_{y} x}}\right] \\
f_{z}=\operatorname{erf}\left[\frac{y+y / 2}{2 \sqrt{\alpha_{y} x}}\right]-\operatorname{erf}\left[\frac{y-y / 2}{2 \sqrt{\alpha_{y} x}}\right]
\end{gathered}
$$

Each of the terms $f_{x}, f_{y}$ and $f_{z}$ range from 0 (if the term accounts for a lot of attenuation) to 2 (if the term does not account for any substantive attenuation). Each term is divided by 2 to determine the contributory impact of the process(es) in attenuating the contaminant concentration. For example, if we want to assume minimal lateral dispersivity we would assume $\alpha_{y}$ is small. At small values of $\alpha_{y}$ the error functions within $\mathrm{f}_{\mathrm{y}} \approx 1$ and -1 and the overall value of $\mathrm{f}_{\mathrm{y}} \approx 2-$ thus $\mathrm{f}_{\mathrm{y}} / 2 \approx 1$.

\section{Return to Section 6.2}




\section{Addendum for Section 6.3 - Mathematical formulation used to evaluate longitudinal dispersion}

Using equation 3 and setting $\mathrm{k}_{\mathrm{s}}=0$ (constant source) and $\mathrm{f}_{\mathrm{y}} / 2=\mathrm{f}_{\mathrm{z}} / 2=1$ we can examine the incremental effects of longitudinal dispersion on the plug flow case presented earlier. The resulting equation is simplified from above and corresponds to the equations for a 1D steadystate plume (developed earlier by Bear (1972) and others):

$$
C_{(x, t)}=C_{0}(1)\left[\frac{f_{x}}{2}\right](1)(1)=\frac{C_{0}}{2} \exp \left[\frac{x\left(1-\sqrt{1+4 \alpha_{x} \lambda / v_{c}}\right)}{2 \alpha_{x}}\right]
$$

Except for the longitudinal dispersivity coefficient, $\alpha_{x}$, the bounding ranges for all of the required parameters are discussed above. Simple techniques to estimate $\alpha_{\mathrm{x}}$ based on various measures of model scale are available from compilations of field data (e.g., Gelhar, 1992).

The simplest of these assume that $\alpha_{\mathrm{x}}$ is a fixed value that is used throughout the plume and that the selected value directly relates to the "plume length" (EPA, 2000). Typically, $\alpha_{\mathrm{x}}=0.1 *$ (plume length) or $\alpha_{\mathrm{x}}=0.1 *$ ((plume length) / 2). This approach provides a good general estimate and is consistent with the derivation of the Domenico solution; however, various investigators have shown that calculating a varying $\alpha_{x}$ throughout the plume as a function of $x$ provides a superior match to field data (Pickens and Grisak, 1981; EPA, 2000). Typically $\alpha_{x}=0.1 *(x)$. While more complex relationships have also been developed (Xu and Eckstein, 1995; Al-Suwaiyan, 1996), a parametric evaluation based on a simple proportionality with distance appears reasonable and was used for our analysis.

\section{Return to Section 6.3}




\section{Addendum for Section 6.4 - Mathematical formulation used to evaluate source decay}

Field data suggest that contaminant sources, even sources of chlorinated solvents, are not constant and inexhaustible (Newell and Adamson, 2005). Using equation 3 and setting $\mathrm{k}_{\mathrm{s}}>0$ (decaying source) and $f_{y} / 2=f_{z} / 2=1$ we can examine the incremental effects of source decay on the longitudinal dispersion case presented above. The resulting equation is:

$$
\begin{gathered}
C_{(x, t)}=C_{0} e^{-k_{s} t}\left[\frac{f_{x}}{2}\right](1)(1)=\frac{C_{0}}{2} e^{-k_{s} t} f_{x} \\
f_{x}=\exp \left[\frac{x\left(1-\sqrt{1+4 \alpha_{x}\left(\lambda-k_{s}\right) / v_{c}}\right)}{2 \alpha_{x}}\right] * \operatorname{erfc}\left[\frac{x-v_{c} t\left(1-\sqrt{1+4 \alpha_{x}\left(\lambda-k_{s}\right) / v_{c}}\right)}{2 \sqrt{\alpha_{x} v_{c} t}}\right]+ \\
\exp \left[\frac{x\left(1+\sqrt{1+4 \alpha_{x}\left(\lambda-k_{s}\right) / v_{c}}\right)}{2 \alpha_{x}}\right] * \operatorname{erfc}\left[\frac{x+v_{c} t\left(1-\sqrt{1+4 \alpha_{x}\left(\lambda-k_{s}\right) / v_{c}}\right)}{2 \sqrt{\alpha_{x} v_{c} t}}\right]
\end{gathered}
$$

Note that while this is still a 1D solution, it is no longer steady-state. The concentrations at any distance are transient - for every $\mathrm{x}$, the concentrations increase and then decrease and the concentrations do not stabilize at a constant value. A reference case for source decay is $\lambda=\mathrm{k}_{\mathrm{s}}$, this allows a reasonable degree of source reduction over time and it falls within the allowable values that do not require complex solutions (Aziz et al., 2002). This scenario is equivalent to some of the cases evaluated for radionuclides (see Burkholder and Rosinger, 1980) and provides a useful parametric demonstration of the impact of source decay. Since the analytical solution is not steady-state, we are now identifying the maximum concentration that is projected for any time at each flow distance. This maximum concentration will occur at different times at each $\mathrm{x}$, but it is this maximum concentration that is the indicator of plume growth/size for purposes of natural attenuation modeling. Examining the time-concentration results for many combinations of parameters (Figure A1), and manipulation of the time dependent equation yielded the following correlation that allows easy prediction of the time for the maximum concentration $\left(\mathrm{t}_{\max }\right)$ at any flow distance (for the special case of $\lambda=\mathrm{k}_{\mathrm{s}}$ ):

$$
t \max =\frac{1.122 *\left(L_{\lambda}\right)^{0.36}(x)^{0.64}}{\left(v_{s} / R\right)}
$$

Note that units in the resulting correlation are consistent. This correlation was developed for a very specific case $\left(\lambda=\mathrm{k}_{\mathrm{s}}\right)$. Note that other formulations are possible. Falta et al., 2005a develop exact analytical solutions for the general problem of an exponentially decaying source concentration (with or without remediation) that feeds a groundwater plume with advection, retardation, and decay, but with no dispersion. Falta et al. (2005a) also develop analytical solutions for the maximum extent of a plume, and for the time of maximum plume extent, both for a decaying source, with or without remediation. A key finding is that the maximum concentration at any $\mathrm{x}$ always occurs when the front first passes by, at a time of $\mathrm{Rx} / \mathrm{v}$ in the absence of dispersion. 


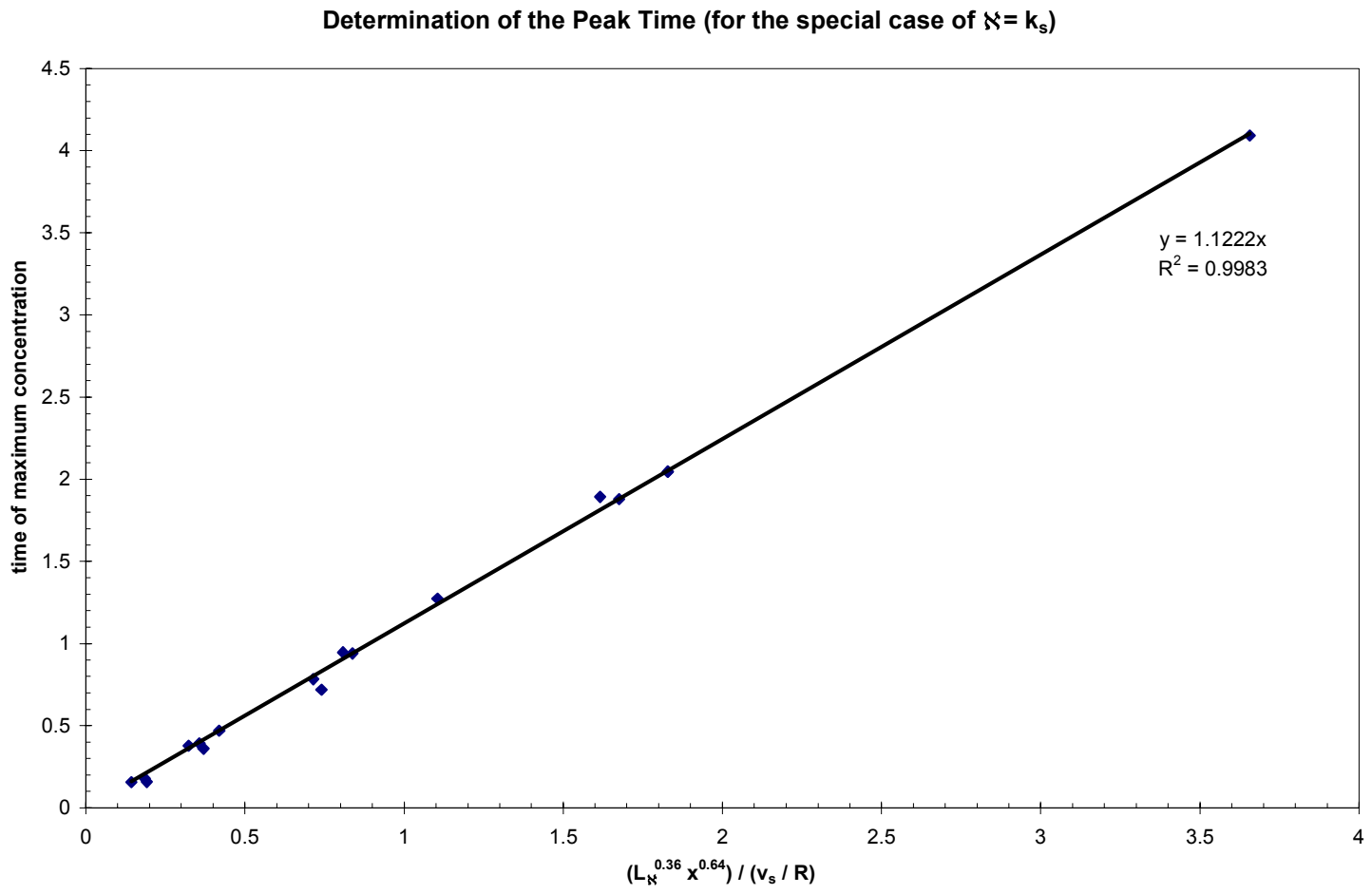

Figure A1. Demonstration of the relationship of $t_{\max }$ to other model parameters for a wide variety of conditions - this is a correlation to the data generated by the Domenico model.

\section{Return to Section 6.4}




\section{Addendum for Section 6.6 - Mathematical formulation used to evaluate transverse dispersion}

An interesting feature of the 3D analytical solution (equation 3), is that the transverse dispersion is decoupled and embodied in the terms $\mathrm{f}_{\mathrm{y}}$ and $\mathrm{f}_{\mathrm{z}}$. Importantly, as shown below, these terms do not include time, flow rate, retardation factor, or degradation rate. They are determined entirely by waste site width, and observation position (x,y and $\mathrm{z}$ ). This has two important ramifications. First, the impact of transverse dispersion can be estimated separately from all of the other factors and applied as an overlay. Second, transverse dispersion can result in plume stability for a given $\mathrm{C} / \mathrm{C}_{0}$ even if there are no degradation mechanisms operating for a site. This suggests that an appropriate parametric evaluation of transverse dispersion would be to evaluate the plume centerline value $\mathrm{f}_{\mathrm{y}}$ and/or $\mathrm{f}_{\mathrm{z}}$ as a function of flow distance for bounding cases of a "small," "medium" "large" sites and then to overlay the multiplier on the previous cases to examine the relative significance of transverse dispersion to the other factors examined above. For transverse dispersion, the small site would represent the "best case," the medium site the "intermediate case," and the large site the "worst case."

For a centerline calculation:

$$
\begin{aligned}
& f_{y}=\operatorname{erf}\left[\frac{Y / 2}{2 \sqrt{\alpha_{y} x}}\right]-\operatorname{erf}\left[\frac{Y / 2}{2 \sqrt{\alpha_{y} x}}\right] \\
& f_{z}=\operatorname{erf}\left[\frac{Z / 2}{2 \sqrt{\alpha_{z} x}}\right]-\operatorname{erf}\left[\frac{Z / 2}{2 \sqrt{\alpha_{z} x}}\right]
\end{aligned}
$$

As with longitudinal dispersivity, the transverse $\left(\alpha_{y}\right)$ and vertical $\left(\alpha_{z}\right)$ dispersivity are typically estimated based on simple correlations with scale, or correlations with longitudinal dispersivity. EPA (2000), based on the high reliability points in Gelhar (1992), suggests that a typical assumption is: $\alpha_{\mathrm{y}}=0.1 * \alpha_{\mathrm{x}}$. For our parametric evaluation we assumed that $\alpha_{\mathrm{x}}=0.1 * \mathrm{x}$; therefore, $\alpha_{\mathrm{y}}$ can be approximated by $\alpha_{\mathrm{y}}=0.01 * \mathrm{x}$. Similarly, EPA (2000), based on EPA (1986), suggests that a typical assumption is: $\alpha_{z}=0.025$ to $0.1 * \alpha_{x}$, but qualifies the suggestion with a recommendation that a low value such as $10^{-99}$ (i.e., neglecting vertical dispersion) is often justified because of vertical constraints in real world aquifers. Thus, the parametric evaluation will be based only on lateral transverse dispersion using the simplified correlation described above.

\section{Return to Section 6.6}


Addendum for Section 6.6 - Graphs of the results for the Medium and Large Site Cases showing the effects of transverse dispersion are on the next two pages. 


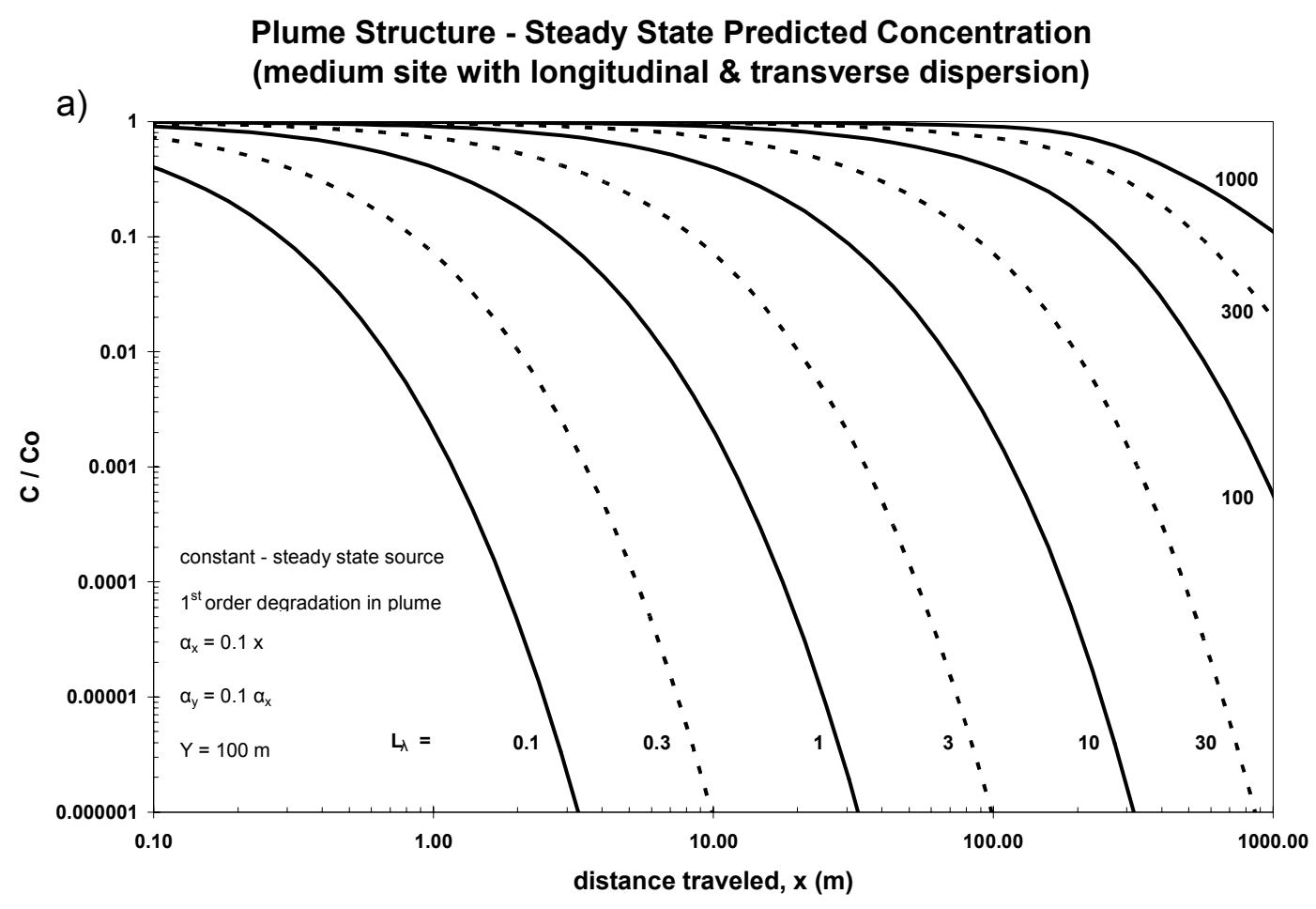

Impact of Transverse Dispersion on Steady State Plume Structure

b) (medium site)

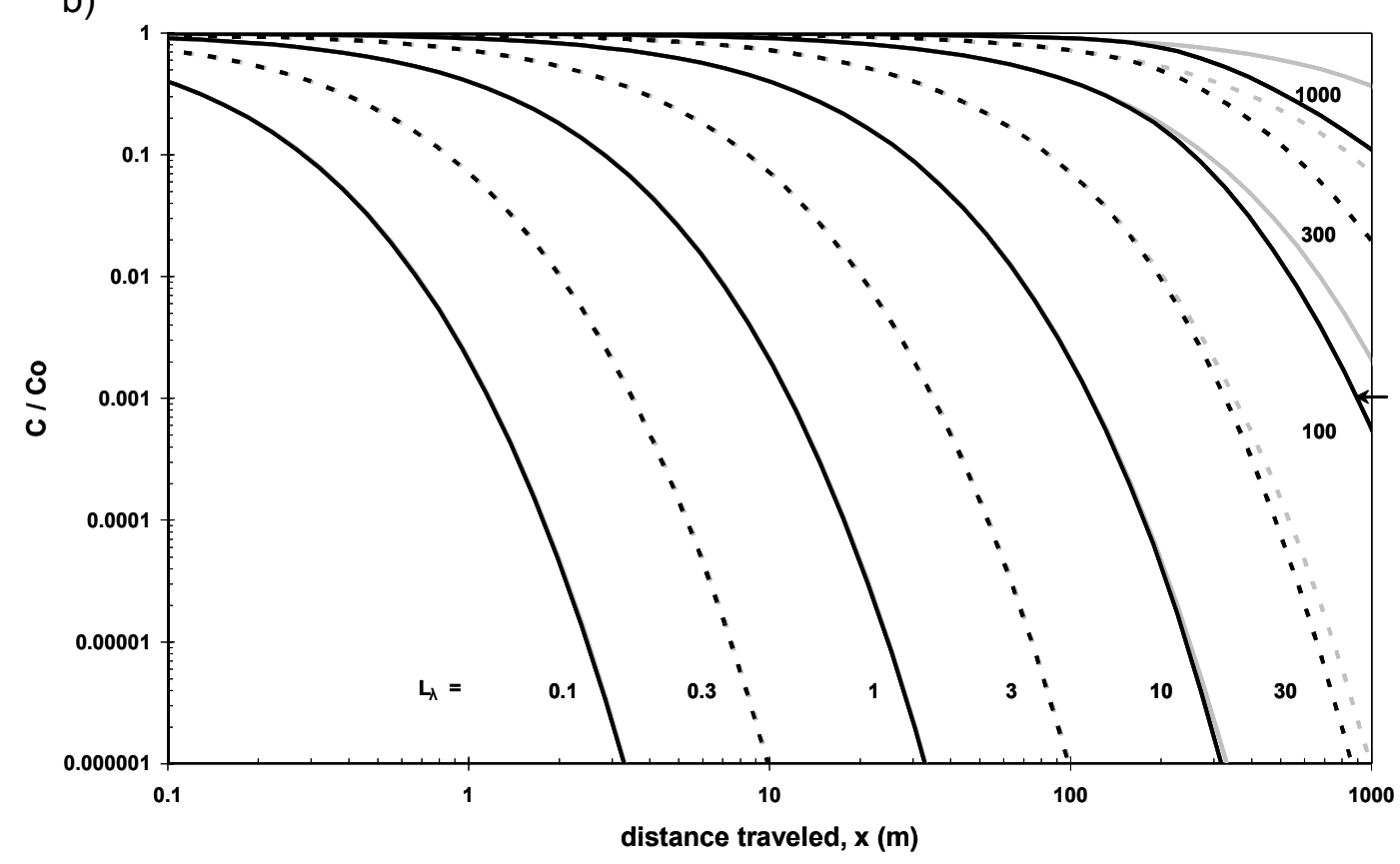

a) general results

b) incremental impact (underlay is 1D steady-state longitudinal dispersion case)

Supplemental Figure. Medium Site -- Overlay of the attenuation by transverse dispersion for a 2D steady-state plume 

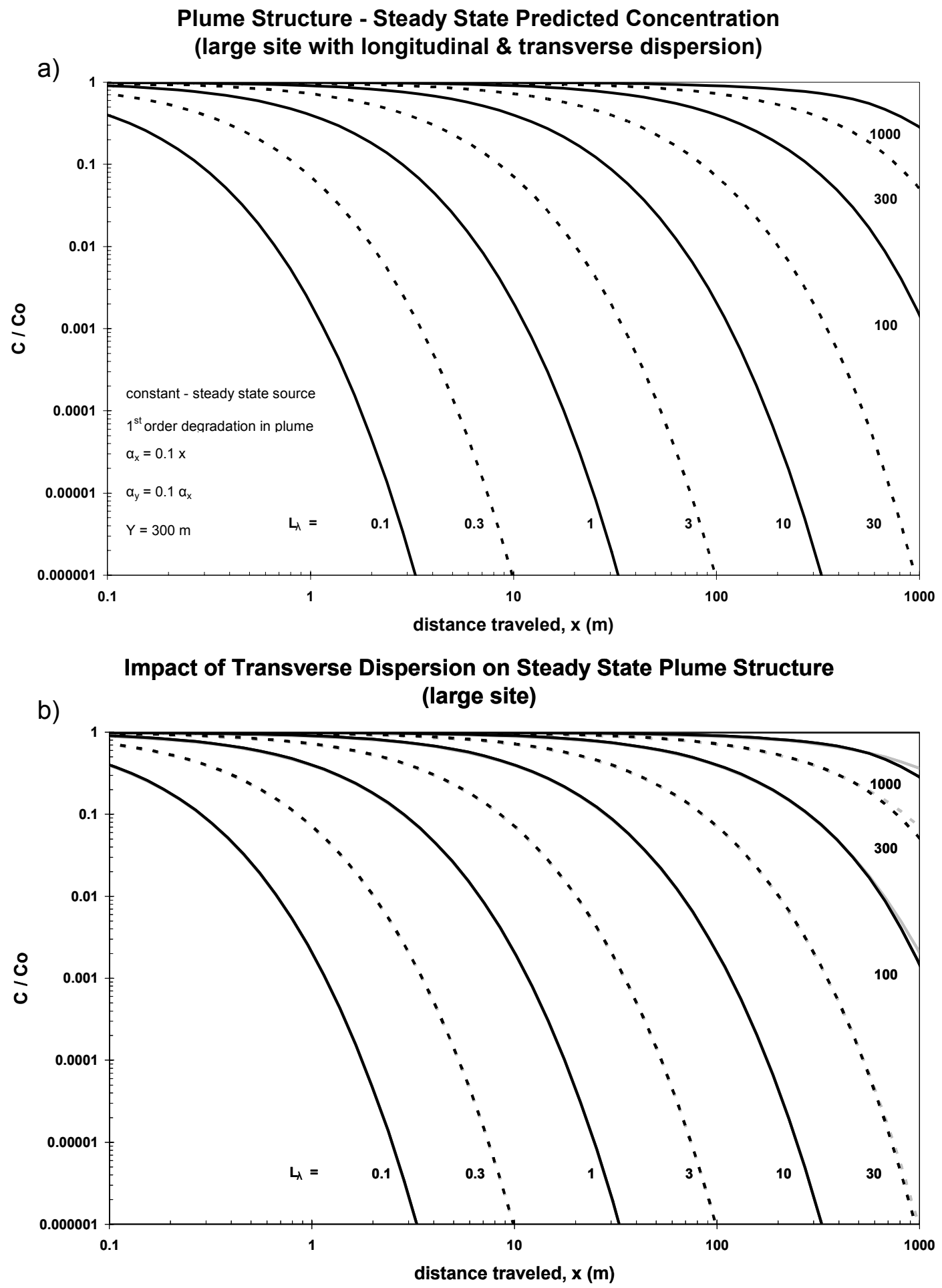

a) general results

b) incremental impact (underlay is $1 \mathrm{D}$ steady-state longitudinal dispersion case)

Supplemental Figure. Large Site -- Overlay of the attenuation by transverse dispersion for a 2D steady-state plume 
WSRC-STI-2006-00082, Rev. 0 June 8, 2006

Page 77 of 77

Return to Section 6.6 